\title{
DEVELOPMENT OF THERMOELECTRIC DEVICES \\ FOR STRUCTURAL COMPOSITES
}

\author{
By \\ Oonnittan Jacob Panachaveettil \\ Bachelor of Science in Mechanical Engineering \\ Mahatma Gandhi University \\ Kerala, India \\ 2007
}

Submitted to the Faculty of the

Graduate College of the

Oklahoma State University

in partial fulfillment of

the requirements for

the Degree of

MASTER OF SCIENCE

December, 2011 


\section{DEVELOPMENT OF THERMOELECTRIC DEVICES FOR STRUCTURAL COMPOSITES}

Thesis Approved:

Dr. Kaan Kalkan

Thesis Adviser

Dr. Ranji Vaidyanathan

Dr. Daryoosh Vashaee

Dr. Khaled A.Sallam

Dr. Sheryl A. Tucker

Dean of the Graduate College 


\section{TABLE OF CONTENTS}

Chapter $\quad$ Page

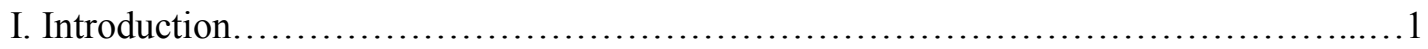

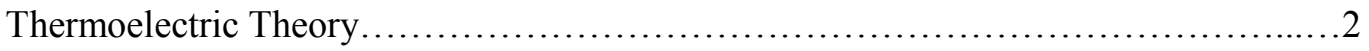

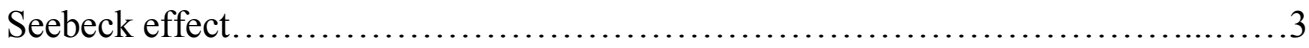

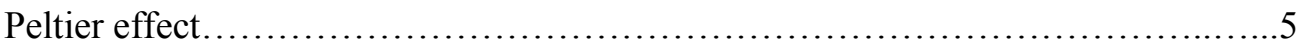

Thermoelectric Figure of merit................................................. 6

Properties of thermoelectric materials..........................................

Thermoelectric structural composites..............................................

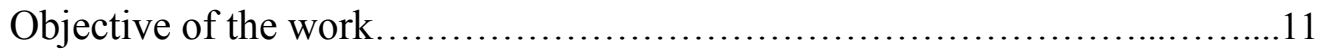

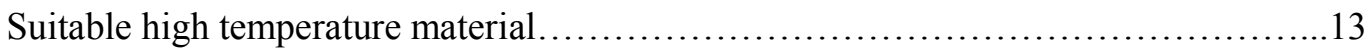

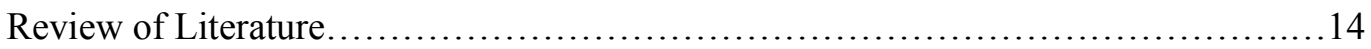

Composites and thermoelectric materials ................................................................... 14

Metal Contacts for thermoelectric materials................................................................. 16

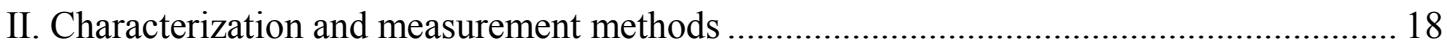

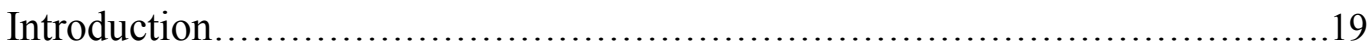

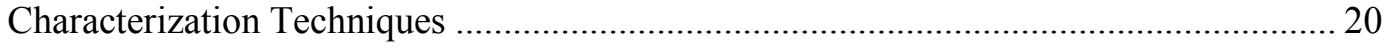

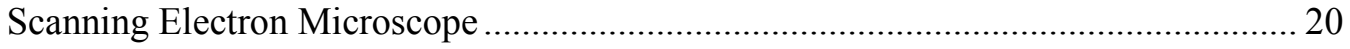

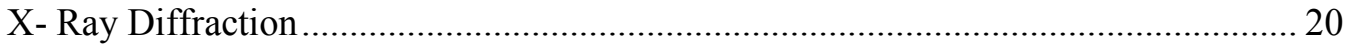

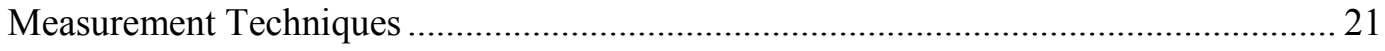

Electrical conductivity and Seebeck coefficient measurement...................................... 21

Thermal conductivity measurements ………………………………………………. 23 
Resistivity measurement using four point probe method for thin films....

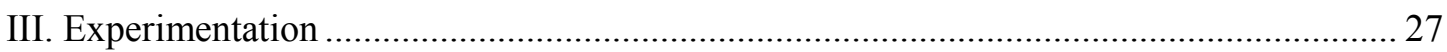

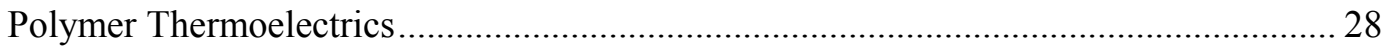

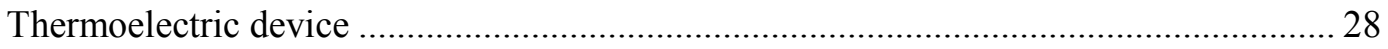

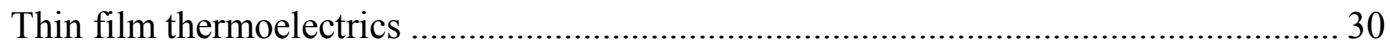

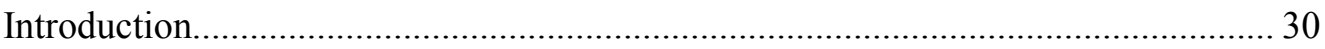

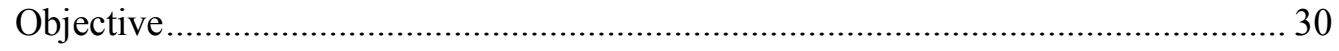

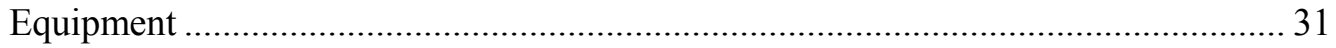

Thermoelectric thin film Experimentation................................................................ 34

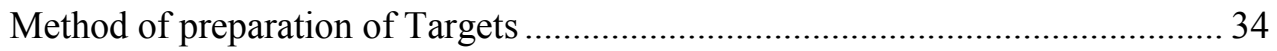

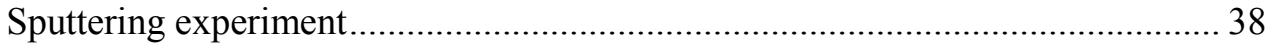

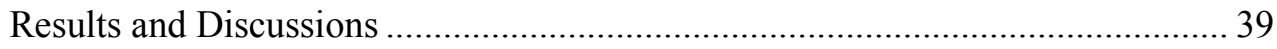

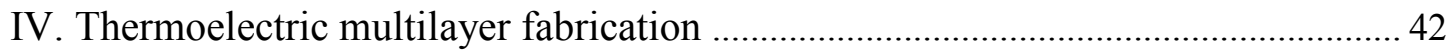

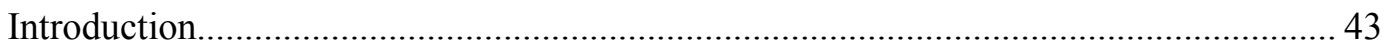

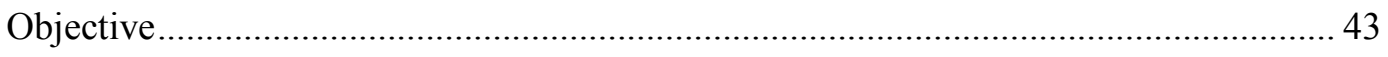

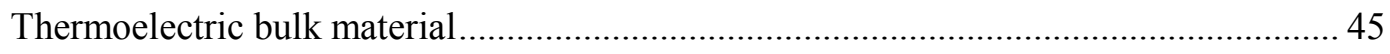

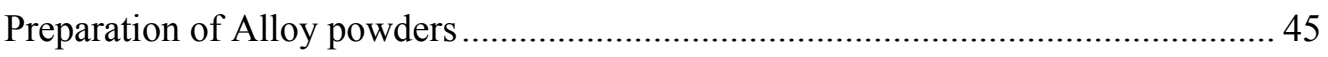

Mechanical Milling and Annealing .............................................................. 46

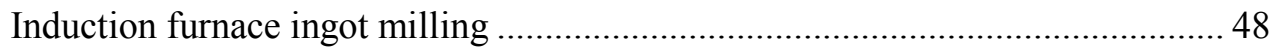

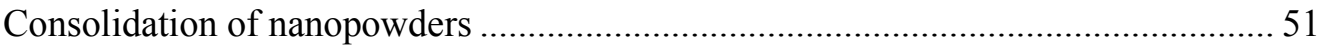

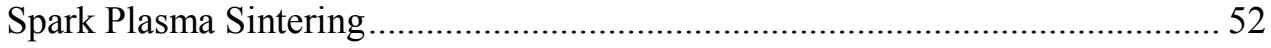

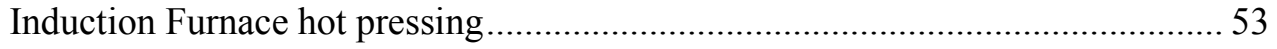

Fabrication of P-type multilayered thermoelectric sample ...................................... 54 


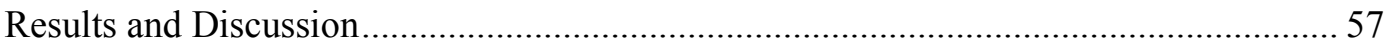

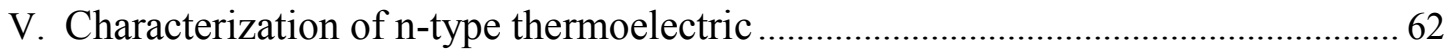

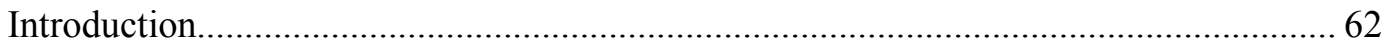

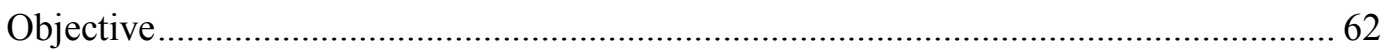

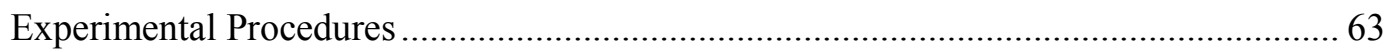

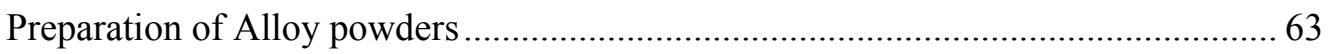

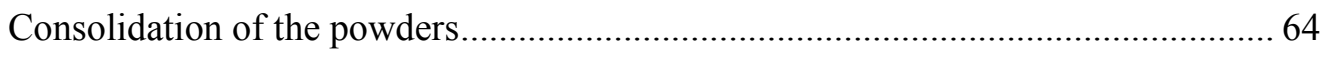

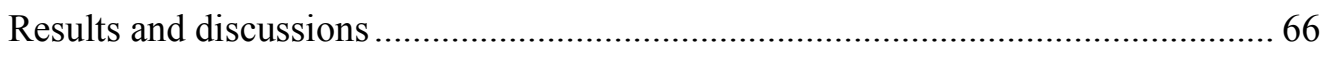

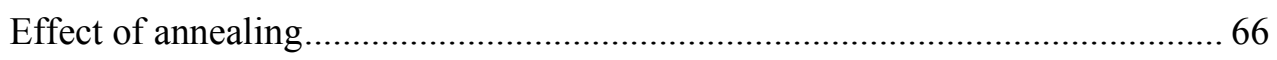

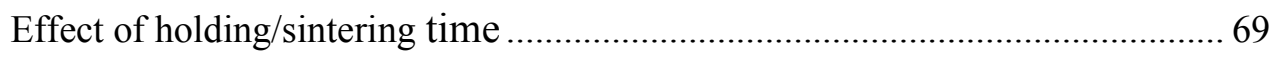

Composite nanoparticle doping with Silicon Germanium Arsenide.........71

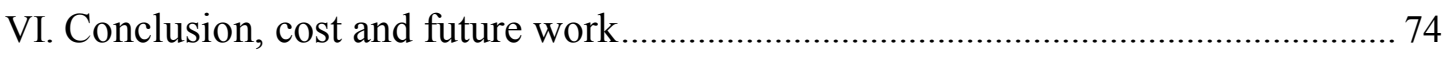

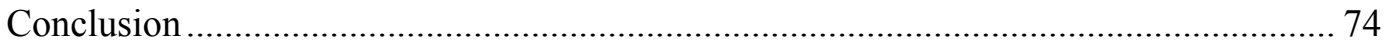

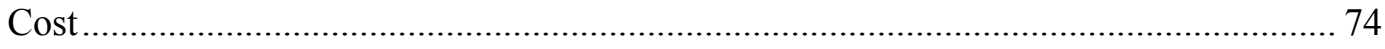

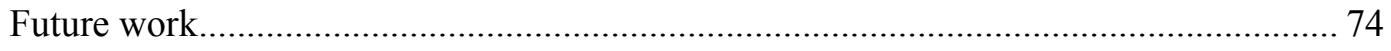

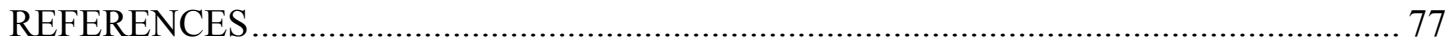

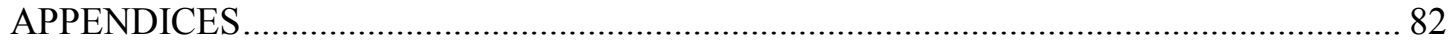




\section{LIST OF TABLES}

Table $\quad$ Page

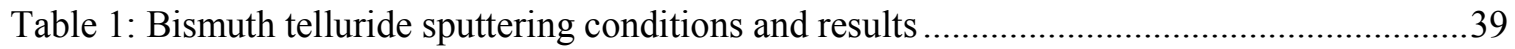

Table 2 : Antimony telluride sputtering conditions and results .................................................4

Table 3: Different doping levels considered for thermoelectric polymer thin films .....................83

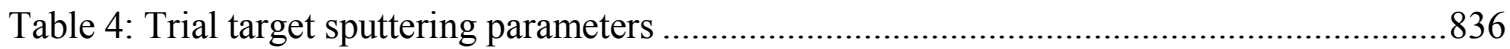




\section{LIST OF FIGURES}

Figure

Page

Figure 1: Seebeck effect using dissimilar metal junctions ...........................................................

Figure 2: Seebeck effect using temperature gradient ..........................................................

Figure 3: Band diagram, density of states greater above Fermi level at the hot junction ................5

Figure 4: Peltier effect, sketch shows the heating and cooling of junctions when electric current is

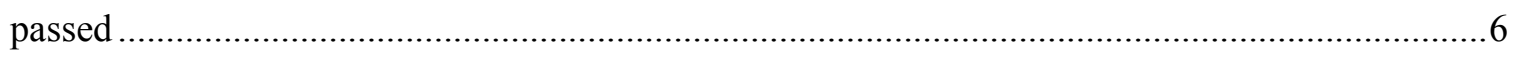

Figure 5: Thermoelectric transport properties as a function of carrier concentration [7] f.............8

Figure 6: Composite prepregs that could be used to make energy harvesting composites ............10

Figure 7: Multi-functional embedded thermoelectric structural composites; thermoelectric module

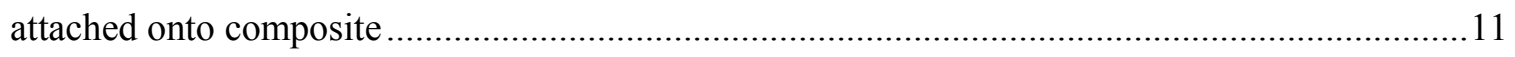

Figure 8: Thermoelectric device; a combination of p-type and n-type thermoelectric material is

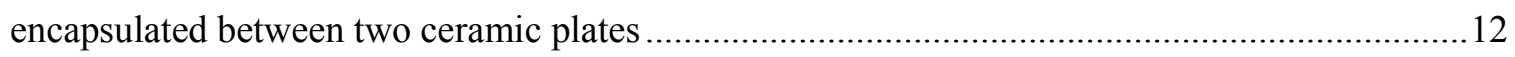

Figure 9: Figure of merit versus temperature for different thermoelectric materials[17] ............14

Figure 10: Schematic of Seebeck-electrical properties measurement system ..............................21

Figure 11: (a) ZEM ...... (b) Sample mounted on ZEM........................................................22

Figure 12: Seebeck coefficient measurement setup, temperature difference exists between the

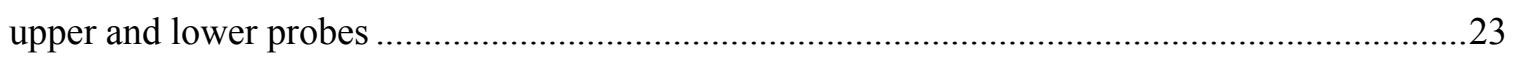

Figure 13: Picture of Netzsch laser flash ....used for thermal conductivity measurements...........24

Figure 14: Schematic of laser flash working principle for thermal conductivity measurement.....25

Figure 15: (a) Schematic, (b) picture of 4-point probe configuration .........................................26

Figure 16: Four probe measurement setup for thin film resistance measurement.........................27 
Figure 17: Schematic of thermoelectric $\mathrm{p}$ - $\mathrm{n}$ junction, with metal contacts and ceramic plates ...29

Figure 18 : AJA Orion Sputtering Equipment used for thin film depositions...............................31

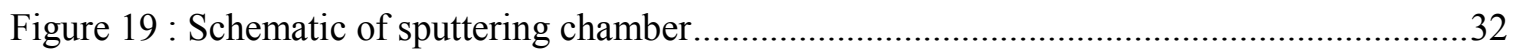

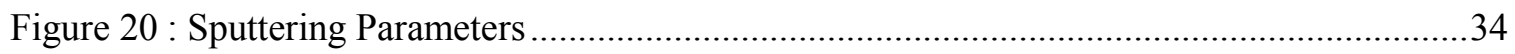

Figure 21 : (a) Induction furnace Schematic (b) Induction furnace experimental setup ................36

Figure 22 : (a) Picture of target after processing and shape formation, (b) Target bonded to copper

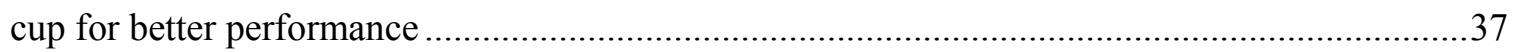

Figure 23: Agglomeration of grains of bismuth telluride sputtered at 60W DC power.................40

Figure 24: (a) Porous film of bismuth telluride sputtered at 50W DC power with no bias, (b) Denser film of bismuth telluride sputtered at 50W DC power with $14 \mathrm{~W}$ RF bias power.............40

Figure 25 : (a) P-type thermoelectric with copper contact on top, (b) n-type thermoelectric with copper contact at bottom, (c) 2-D image showing the cross-section of module ...........................44

Figure 26 : Cross-section of p-type thermoelectric; Schematic of 2 inch sample (inset)...............45

Figure 27 : (a) High energy mill, (b) Stainless steel jar used for alloying the elemental powders 47

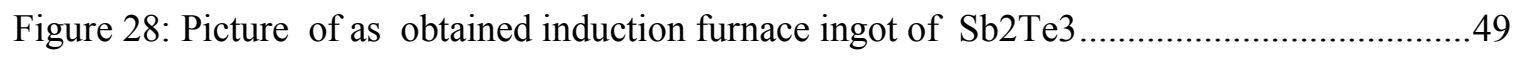

Figure 29 : SEM image of final alloy powder used for experiments ..........................................50

Figure 30: XRD plot of the alloyed powders used for experiments ...........................................50

Figure 31 : (a) Alloyed powders ready to be pressed and sintered, (b and c) Sample obtained after

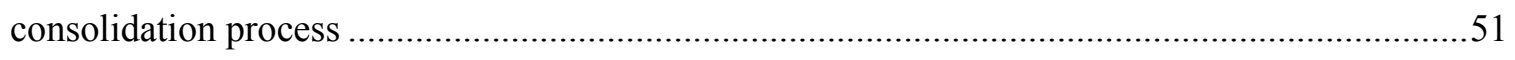

Figure 32: Schematic Spark plasma sintering setup used for alloy powder compaction ...............52

Figure 33 : Schematic of induction furnace hot pressing ........................................................53

Figure 34 : Picture of powder loaded graphite die loaded with stainless steel rod, the arrows represent the shearing motion needed for flattening the powders ..............................................55

Figure 35: Picture of different powder layers after flattening, compaction and cold pressing, (a) Copper, (b) Nickel, (c) Thermoelectric powders ..................................................................56

Figure 36: Cross-section of a sandwich structure pressed................................57 
Figure 37 : Temperature dependence of (a) electrical conductivity, (b) Seebeck coefficient, (c) power factor, (d) thermal conductivity and (e) figure of merit of crystalline industrial sample and multilayered thermoelectric sample .58

Figure 38: Microscopic image of interface; (a) Discoloration due to Copper diffusion layer into thermoelectric (b) Multilayered structure composite with Nickel diffusion barrier included (TE = Thermoelectric) .59

Figure 39 : SEM image of the interface regions, spherical shape Ni can be observed .60

Figure 40: Graphic representation of the EDS data showing diffusion of the layers 61

Figure 41: Flowchart showing powder alloying and sample pressing conditions .65

Figure 42: Temperature dependence of (a) electrical conductivity, (b) Seebeck coefficient, (c) power factor, (d) thermal conductivity and (e) figure of merit of different powders and sample annealing time

Figure 43 : Temperature dependence of (a) electrical conductivity, (b) Seebeck coefficient and (c) power factor (d) thermal conductivity and (e) figure of merit of different samples showing effect of holding/sintering time, pressure and temperature .70 Figure 44: : Temperature dependence of (a) electrical conductivity, (b) Seebeck coefficient, (c) power factor, (d) thermal conductivity and (e) figure of merit of different powders and sample annealing time .72

Figure 45 : Picture of film dried on glass plate .84 


\section{CHAPTER I}

\section{INTRODUCTION}

In the current scenario of global energy crisis, energy harvesting is very important. Although, ample amount of energy can be generated to cater to most of the energy needs, this energy is obtained from non-renewable sources [1]. Fossil fuels, which are non - renewable sources of energy are getting depleted fast as per person consumption of energy is increasing on a daily basis. Moreover, the fact of population explosion is also a contributing factor to the problem [2]. Fossil fuel days are counted and alternate sources of energy production have to be explored [3].

The method of energy harvesting and efficient conversion without the potential depletion of any other resources is the need of the hour. From the very beginning, the inherent problem with the fossil fuels, more than it being a non-renewable source of energy, it was highly polluting [4]. Therefore, green energy possibilities have to be considered seriously. Increased consumption and dependence on fossil fuels deplete the natural resources and more importantly increase pollution. Air pollution leads to global warming [5].

In order to overcome these problems, the solution is to efficiently manage the energy. A slow and smooth transition to the green energy world is imperative, judging by the global energy requirements and environmental crisis now. Nothing can be more appealing than the thought, realization and implementation of a green source of energy that is abundant and can be tapped 
with absolute ease. Thermal energy is a very attractive source of energy with many advantages [6]. There is always unused, waste heat energy produced from home heating, automotive exhaust, and industrial processes [7]. Temperature difference around surroundings, if efficiently captured can lead to energy harvesting.

Thermoelectrics is the science of conversion of thermal energy into electrical energy and vice versa [8]. This phenomenon was first discovered by Seebeck in 1821 [9]. Seebeck effect, the concept of production of energy utilizing the temperature difference between two surfaces is a big potential field of alternative and pollution free energy source. This concept has been around since last century, recently they have gained much attention [10].

Thermoelectrics is a revolutionizing field because of its many advantages over the conventional energy harvesting methods even though the efficacy of these devices are very low and they are expensive. Thermoelectric devices are green sources of energy. They do not produce emissions and no byproducts are created because of their operation, they do not have any moving parts and are silent [11]. No maintenance, high reliability and long lifetime are many of the common questions that are to be addressed, if this technology is to be used. Present thermoelectric devices satisfy all these parameters to limited extent.

Another enormous advantage is that thermoelectric technology can be used to create both big and miniature devices, which are very impactful, especially in this nanotechnology era.

\subsection{Thermoelectric Theory}

To better understand the science behind thermoelectric, basic concepts are discussed in the following sections. When two junctions of dissimilar metals are subjected to a temperature difference, voltage is produced. The vice versa is also true, when voltage is applied to two dissimilar metals, a temperature difference in developed in the two joining junctions. These are entirely different concepts and they have different applications in the thermoelectric field. 


\subsubsection{Seebeck effect}

When two dissimilar conductors are joined together and a temperature gradient exits between two junctions, an electric field is created, this is known as Seebeck effect. A schematic to better understand the Seebeck effect is shown in Figure 1. A and B are two dissimilar metals which are connected together and the junctions ( $\mathrm{J} 1$ and $\mathrm{J} 2$ ) are subjected to a temperature difference, resulting in a net voltage generation. One junction will be at a higher temperature relative to other, thus one junction will be hot junction and other cold.

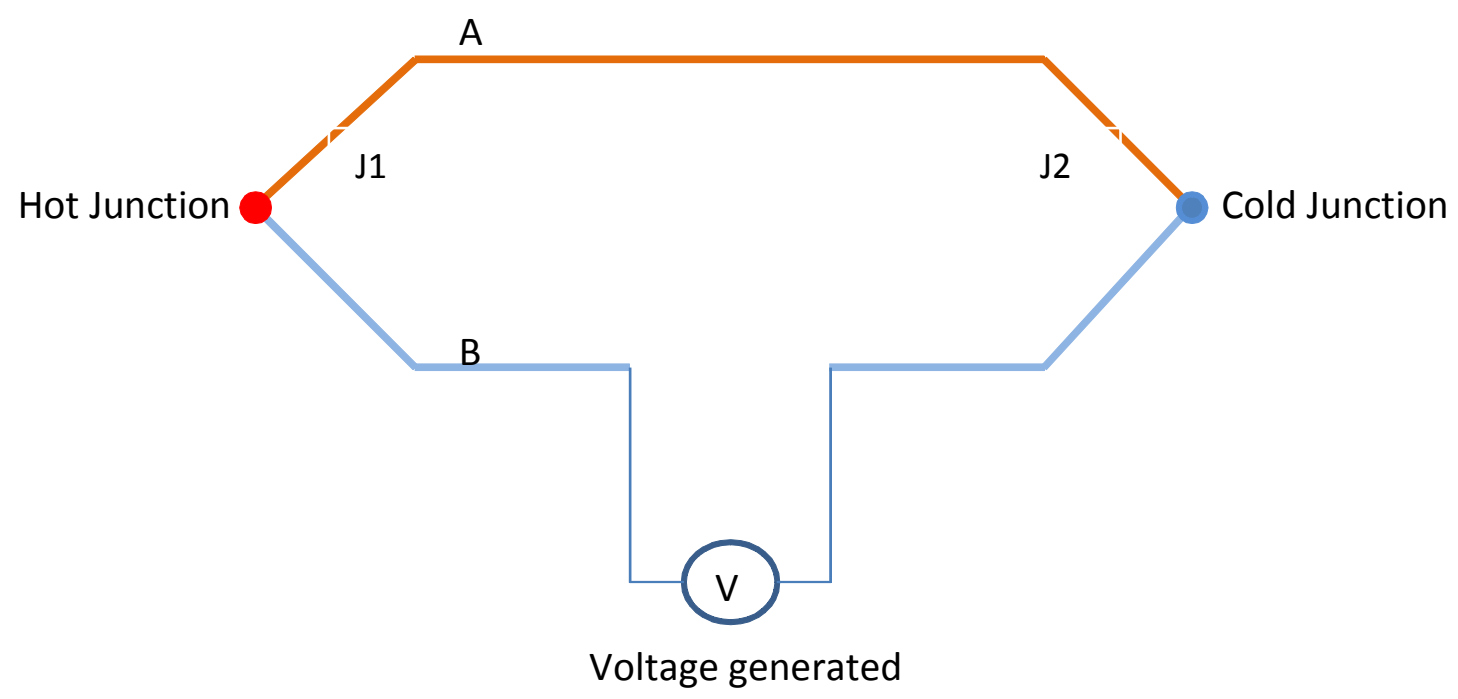

Figure 1: Seebeck effect using dissimilar metal junctions

Such a single unit junction, known as a thermocouple will produce a very small voltage. However connecting many of these thermocouples in an electrically series fashion will lead to a corresponding increase in the voltage and known as thermopiles. Thermopiles are extensively used in thermoelectric applications. They find application in wide range of fields from power generation in satellites, engine exhaust temperature recovery in jet engines and cars to thermoelectric watches.

Seebeck effect is also a property of individual bulk materials, schematic shown in Figure 2, although the effect is prominently observed in dissimilar junctions. This concept has been used to 
measure temperatures and also is the principle behind power generation. The ratio of voltage generated because of the temperature gradient, to the temperature difference is called Seebeck coefficient and given by equation 1.1 .

$$
S=-\frac{d V}{d T}
$$

Where $\quad \mathrm{S}$ - Seebeck coefficient $(\mathrm{V} / \mathrm{K}$ or $\mu \mathrm{V} / \mathrm{K})$

V - Voltage (Volts)

T - Temperature (Kelvin)

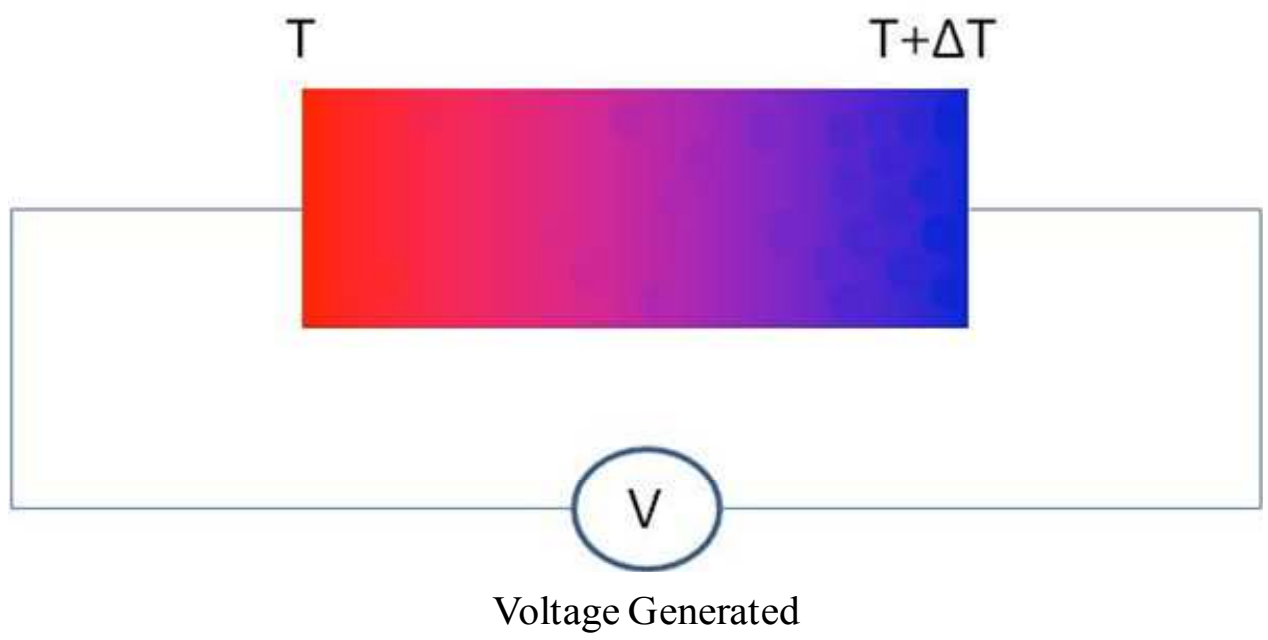

Figure 2: Seebeck effect using temperature gradient

The unit dimension of the Seebeck coefficient is volt per degree, but it is usually expressed in micro-volts per degree Kelvin $(\mu \mathrm{V} / \mathrm{K})$. The sign of S is positive for a $\mathrm{p}$-type material and negative for an n-type material.

Simple explanation of the physics happening during the Seebeck effect can be summarized below. Voltage is developed due to flow of charge through a circuit. The charge carriers in this case are positively charged holes and negatively charged electrons. At the junction subjected to relative higher temperature, energy possessed by the carriers are high compared to the junction at lower temperature. The holes and electrons at higher temperature junction attain more kinetic 
energy and tend to travel to a lower energy state. Therefore, net diffusion of carriers from one side to another can be observed, until the equilibrium energy state is achieved [12]. The density of state of carriers above the Fermi level, at the higher temperature side will be greater [13]. This portrayed in the band energy diagram in Figure 3.

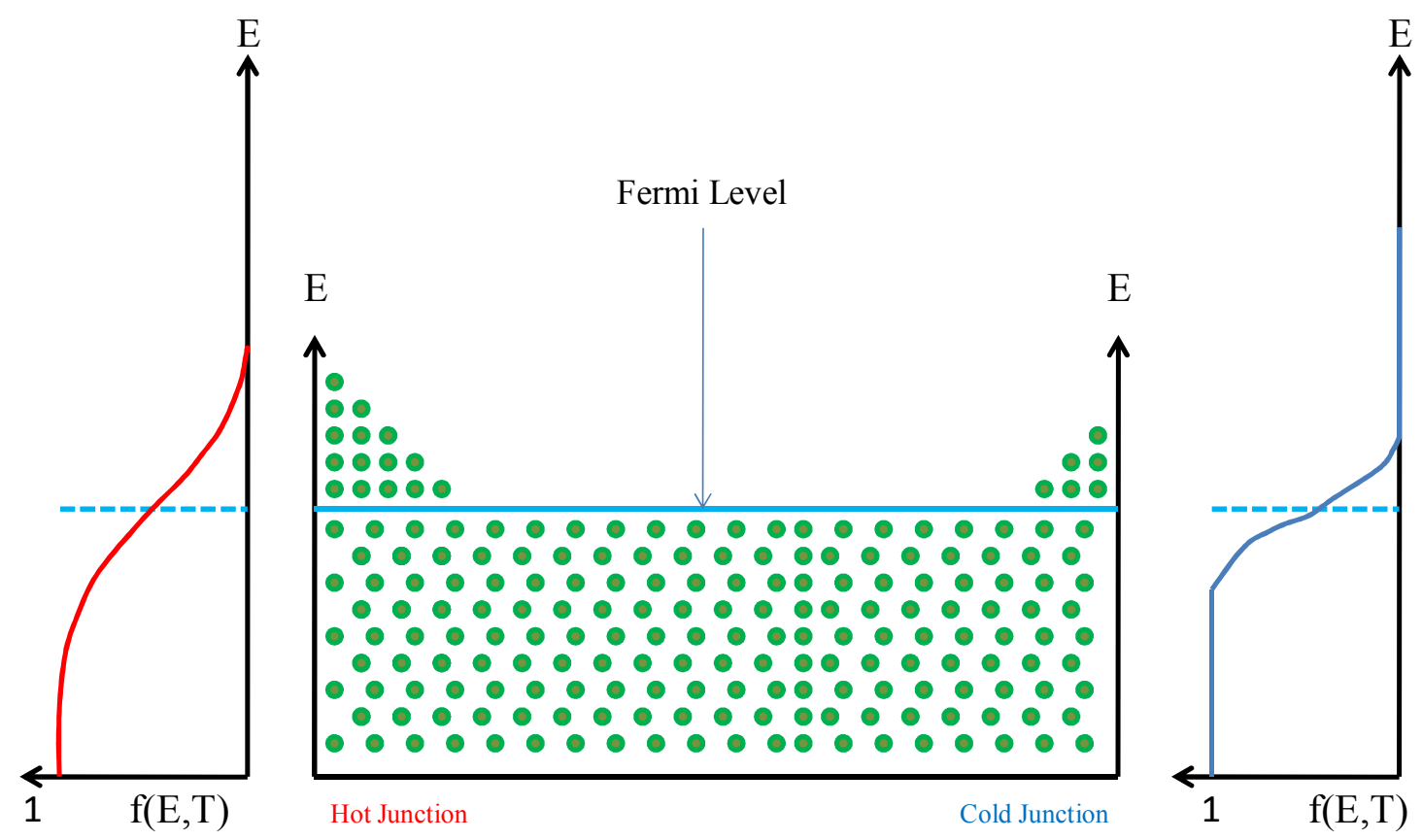

Figure 3: Band diagram, density of states greater above Fermi level at the hot junction

\subsubsection{Peltier effect}

The reverse of the Seebeck effect is the Peltier effect. Discovered by Peltier in 1834 [8], it is another principle concept of thermoelectrics widely studied and efficiently put into action. Peltier effect states that when electric current passed through two dissimilar metal junctions, a temperature gradient is induced between the two junctions, a schematic is shown in Figure 4. This concept is also valid for single bulk materials. 


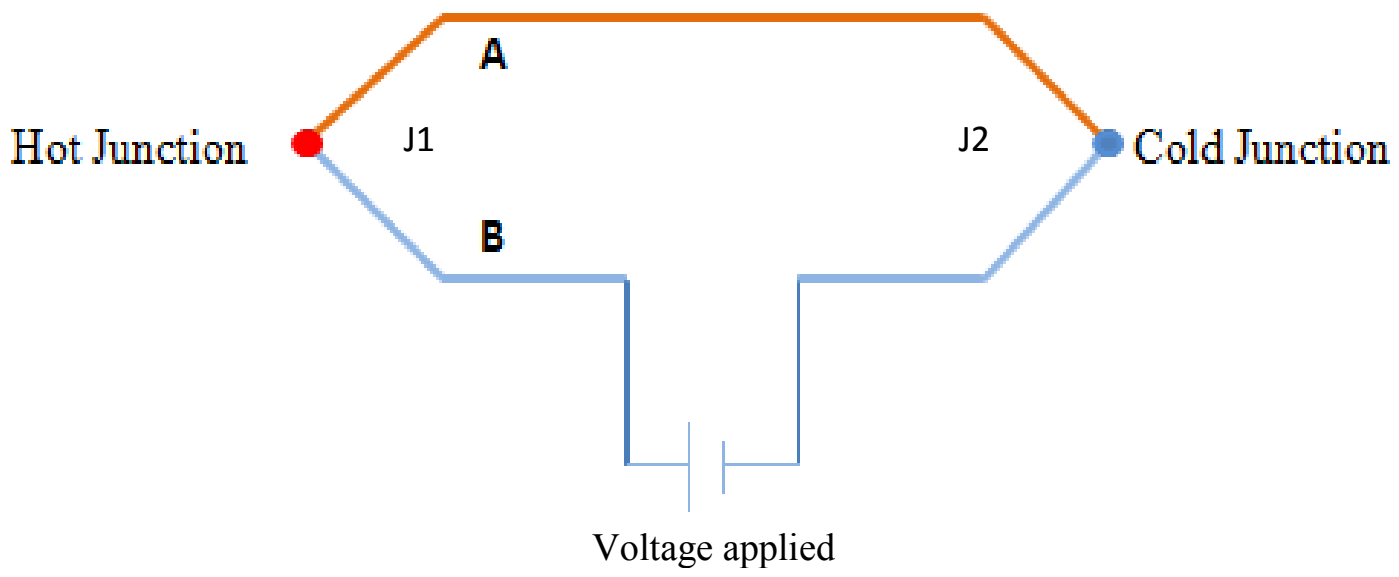

Figure 4: Peltier effect, sketch shows the heating and cooling of junctions when electric current is passed

Applications of Peltier effect ranges from thermoelectrics refrigerators, heated seating in automobile to cooling systems in electronics. Computer microchips and processors which develop a large amount of heat over extended period of time, are cooled using the thermoelectric devices, employing the Peltier effect.

Thermoelectrics can be used for two applications, either for power generation or for temperature control by employing the Seebeck effect or Peltier effect respectively.

\subsubsection{Thermoelectric Figure of merit}

All thermoelectric materials and devices are characterized by a dimensionless quantity called "Figure of Merit (ZT)".

$$
Z \mathrm{~T}=\left(\frac{\mathrm{S}^{2} \sigma}{\mathrm{k}}\right) \mathrm{T}
$$

Where S - Seebeck coefficient (Volt/Kelvin or $\mu \mathrm{V} / \mathrm{K}$ )

$\sigma$ - Electrical conductivity (Siemens/meter, $\mathrm{S} / \mathrm{m}$ )

k - Thermal conductivity (Watt / Kelvin, W/K)

$\mathrm{T}$ - Average temperature of the two junctions 
From the above relation it can be observed that figure of merit is directly proportional to Seebeck coefficient (S), electrical conductivity $(\sigma)$ and inversely proportional to thermal conductivity $(\mathrm{k})$. The efficiency of the thermoelectric materials or the device increases with an increase of $Z$. The product of squared Seebeck coefficient and electrical conductivity is known as power factor (PF). Seebeck coefficient, electrical conductivity and thermal conductivity are the thermoelectric transport parameters/ properties. The value of $\mathrm{Z}$ can be increased by increasing Seebeck coefficient and electrical conductivity, at the mean time lowering the thermal conductivity. There is a limit to which we can do this alteration. Physically, beyond an optimum value we cannot increase $\mathrm{Z}$, as the thermal conductivity also increases with increase in electrical conductivity. Since figure of merit is a product of $\mathrm{Z}$ and temperature $(\mathrm{T})$. Temperature is a crucial factor at which all the parameters affecting $\mathrm{Z}$ are measured. A value of $\mathrm{ZT}=4$ corresponds to an efficiency of $40 \%$ relative to Carnot efficiency.

\subsubsection{Properties of thermoelectric materials}

Altering all the thermoelectric properties/parameters in the favorable direction is tough, when we are dealing with bulk materials, since they are all interdependent on each other. Semiconductors are the best materials for thermoelectric proposes, since we can efficiently alter the material properties to cater to best thermoelectric conditions. Figure 5 shows a plot of the carrier concentrations of different materials verses thermoelectric transport properties. The rationale of failure to use metals and insulators for thermoelectric applications can be inferred from the plot. Insulators have a high Seebeck coefficient and low thermal conductivity but have very poor electrical conductivity, giving rise to lower ZT. On the contrary, metals have good electrical conductivity because of the presence of large amount of carriers, but this is at the expense of the thermal conductivity. Greater carriers for electrical conductivity would imply greater thermal 
conductivity as the carriers both holes and electrons are good conductors of heat. Moreover, carrier concentration is inversely proportional to Seebeck coefficient, making metals unfit for thermoelectric applications.

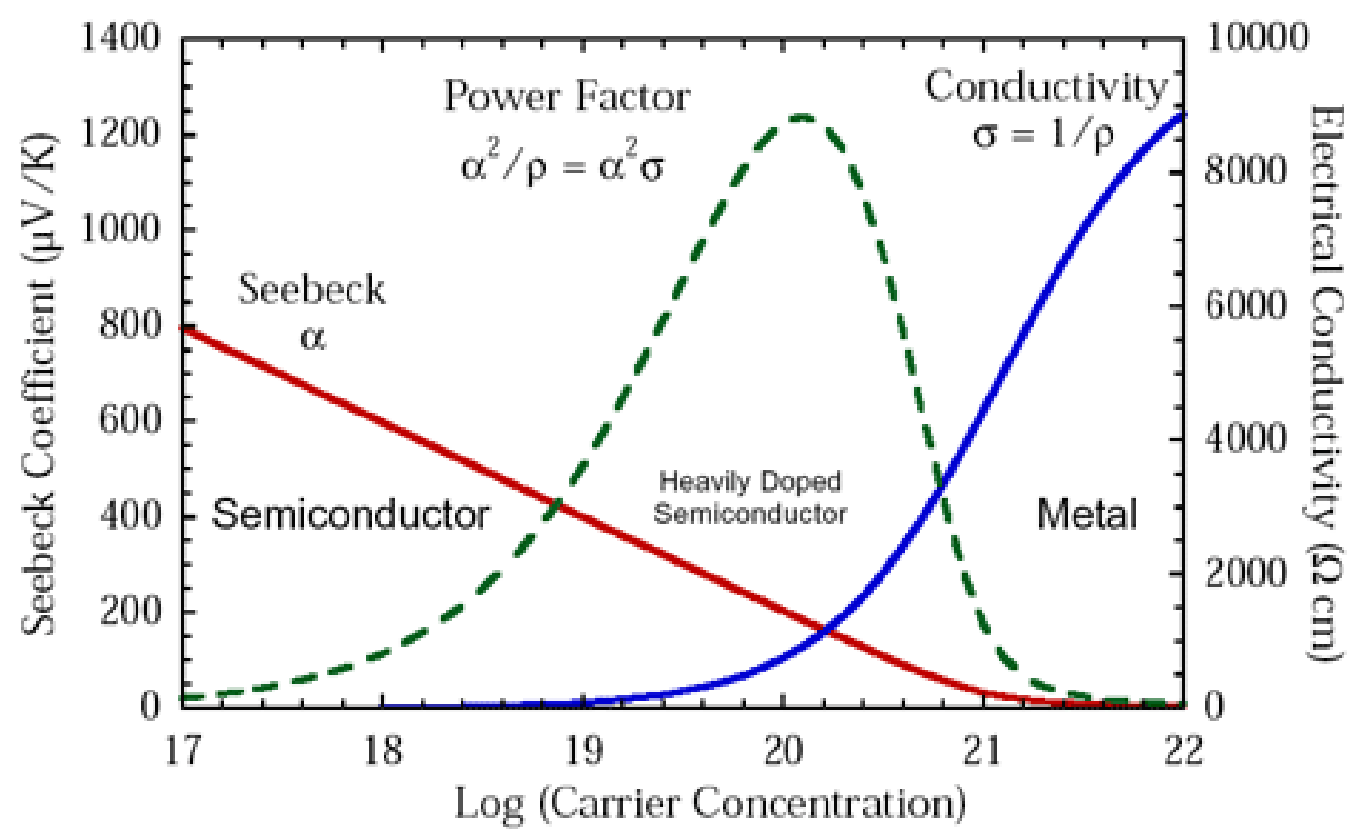

Figure 5: Thermoelectric transport properties as a function of carrier concentration [7]

However, in semiconductors, carrier concentrations can be varied, by varying the amount of doping concentration. Reasonable electrical conductivity can be achieved, without substantially affecting thermal conductivity by doping semiconductors. Altering the concentration of charge carriers, either the number of the holes or electrons, favorable electrical conductivity values as close as in metals are possible.

Depending on the increase of the carrier type, semiconductor materials can be classified as either n-type or p-type materials. Increasing the amount of electron charge carriers compared to hole will result in n-type. Similarly, increasing the amount of holes compared to electrons will result in p-type semiconductor. Thermoelectric devices or modules are combination of both p-type and n-type materials to obtain the maximum efficiency and ZT values. 


\subsection{Thermoelectric structural composites}

Fossil fuel depletion is not far away, it is about time to give up dependence on fossil fuels. Tapping into the nature reserve for the last century and ever increasing pollution problems, an expedite solution for solving dependence on the fossil fuel is critical. In the contemporary world of energy crisis, energy harvesting is very crucial.

Contemplating along these lines, the idea is to make energy harvesting composites. For this purpose of energy harvesting, temperature difference between two surfaces can be utilized. By incorporating thermoelectric devices onto structural composite materials energy could be harvested during the service of the components. Merging thermoelectrics and composite technology gives rise to a very novel energy harvesting method, compared to the conventional approach. A structural material like composite with embedded thermoelectric device will have the energy harvesting capability in addition to the integral structural role, making them "Multifunctional embedded thermoelectric structural composites". Composites as the name signifies contain a combination of two completely different phases matrix and fiber phase. The matrix phase can be considered to be a paste/resin material which binds together the fiber phases. Fibers are dispersed in the matrix phase. The fiber phase is the load carrying component in the composite, whereas the matrix can be considered to be the load transfer phase. Load applied on the composite is first received by the matrix phase and transferred to the fiber phase. Owing to their high specific strength, fatigue and environmental resistance, they find wide structural applications in construction, aerospace and automotive industry. Figure 6 shows the picture of a composite prepreg that could be used to mount the thermoelectric devices on. 


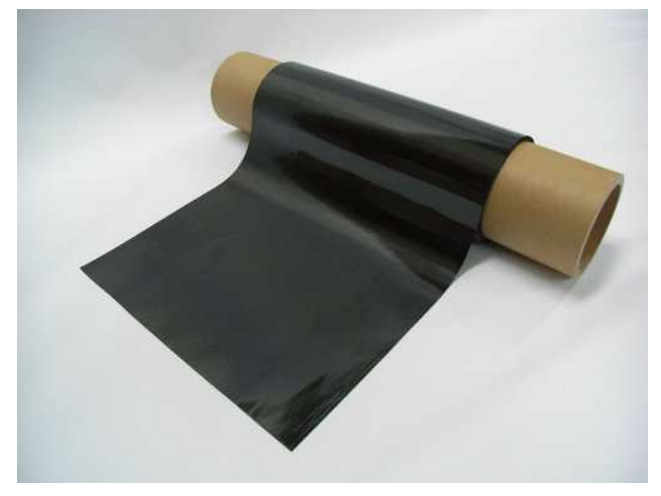

Figure 6: Composite prepregs that could be used to make energy harvesting composites

These multi-functional embedded thermoelectric structural composites can be used to replace several existing structural components. New structures and panels employing regular composites can be replaced with multi-functional embedded thermoelectric structural composites, satiating both the structural application and energy harvesting need. One major field of application of these multifunctional thermoelectric structural composites are sound barriers on freeways. Current RCC (Reinforced Cement Concrete) structures can be replaced with multi-functional embedded thermoelectric structural composites, which in addition to energy harvesting capabilities and structural integrity, can be made to absorb sound disturbances, thus act as acoustic absorbers.

Another potential field of application is in energy harvesting composite panels. They can be made into thermoelectric panels for houses and industries. There exists a large temperature difference between surfaces at homes and industries, which can be utilized to generate thermoelectric energy. Further modification to form flexible thermoelectric composite panels like Dow Corning's "Powerhouse" flexible photovoltaic shingles can be conceived [14]. These shingles in addition to protecting the roof of the house from external environment, also has the ability to generate energy from solar power. Photovoltaics and thermoelectrics are very closely related fields, since, both of these fields deal with semiconductors. 


\subsubsection{Objective of the work}

The project aims to fabricate efficient thermoelectric device to incorporate on the composite. Initially, it was proposed to make a thermoelectric polymer film to be incorporated into composites. Polymer based thermoelectric approach did not prove very promising. The objective of incorporating the polymer films was replaced to produce thermoelectric devices to be attached onto composites. Shown in Figure 7 is the proposed end product of the project. Thick film and thin film were preferred as thermoelectric materials, compared to the thick bulk, because of additional control over low dimensional materials following the prediction of Dresselhaus et. al.[15] moreover the contact resistance is also reduced. Several of these devices will be placed on a composite surface.

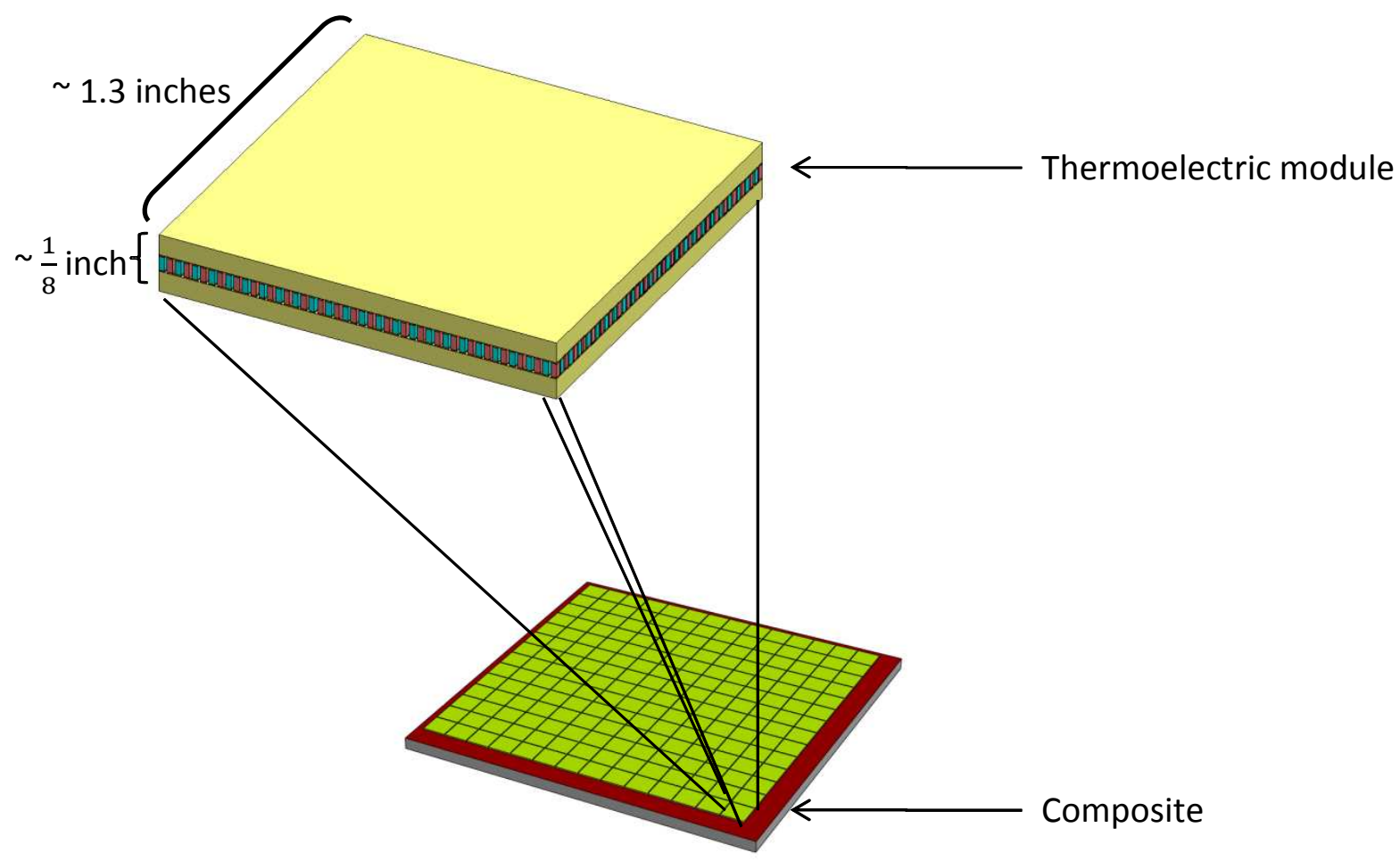

Figure 7: Multi-functional embedded thermoelectric structural composites; thermoelectric module attached onto composite 
Suitable thermoelectric materials consisting of p-type and n-type will be used to fabricate device of about 1.3 inch by 1.3 inch and 0.078 inch $(2 \mathrm{~mm})$ thick. P-type and n-type materials fabricated will be processed, merged to form final thermoelectric device which will be housed in between a silicon wafers or ceramic substrates. A layered breakup of the proposed thermoelectric device is shown in Figure 8 .
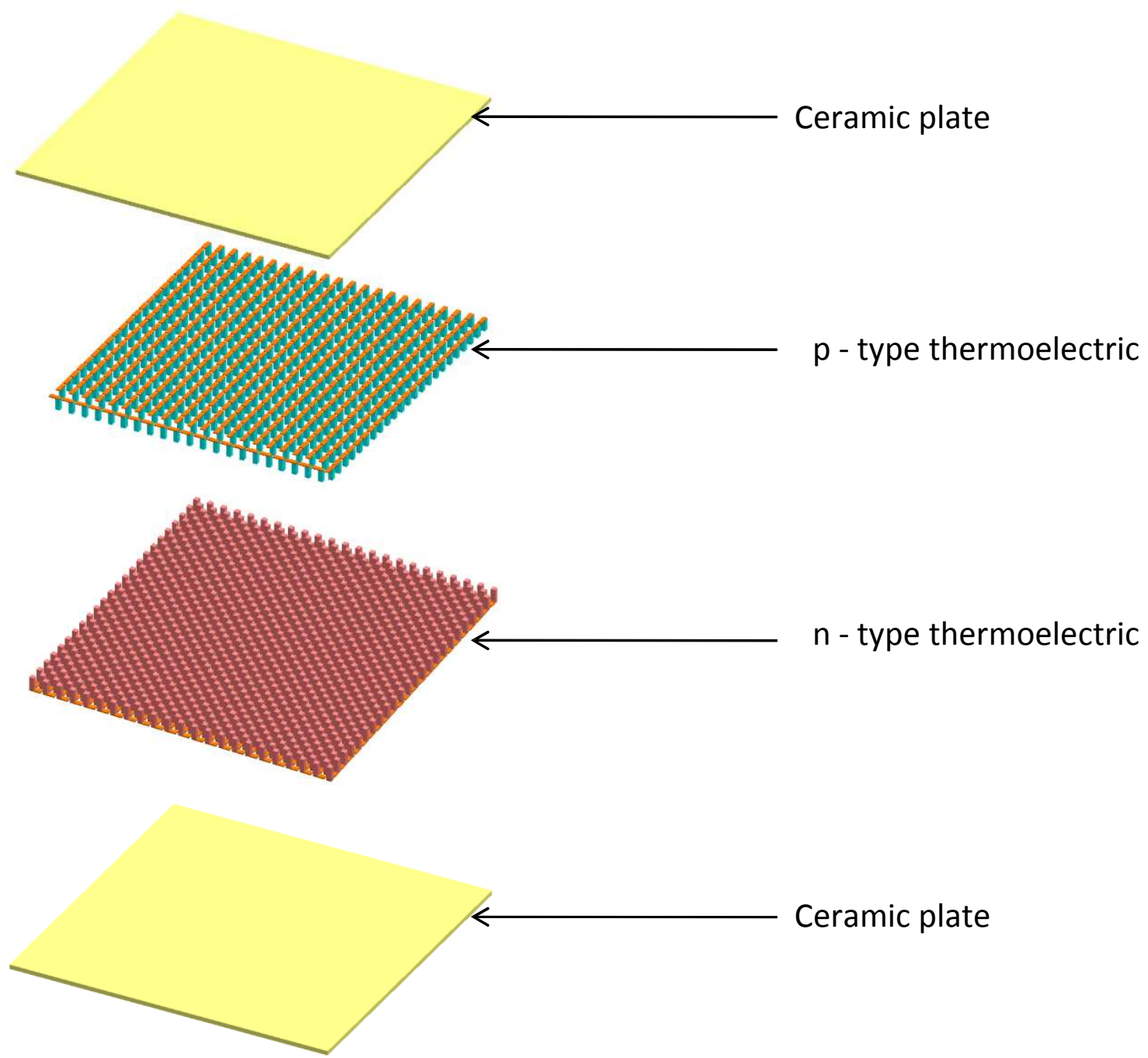

Figure 8: Thermoelectric device; a combination of p-type and n-type thermoelectric material is encapsulated between two ceramic plates 


\subsection{Suitable high temperature material}

Many thermoelectric materials can be used for thermal energy harvesting applications. Each material is specified for use at particular temperature range. Since thermoelectric power can be harvested from high temperature sources like jet engine exhaust $\left(1100^{\circ} \mathrm{C}-1700^{\circ} \mathrm{C}\right)$ to around room temperature applications $\left(25^{\circ} \mathrm{C}-300^{\circ} \mathrm{C}\right)$. There is a wide range of thermoelectric materials to select from.

Multi-functional embedded thermoelectric structural composites, deals with low temperature applications, tapping heat energy in the range from $30^{\circ} \mathrm{C}$ to $150^{\circ} \mathrm{C}$. Best materials for these temperature ranges are alloys of bismuth telluride. Figure 9 shows a plot of the figure of merit versus temperature of different materials. Bismuth telluride and alloys display the maximum ZT at low temperature when compared to other materials. The best materials to use for near room temperature applications, like refrigeration and power generation are bismuth antimony telluride and bismuth selenium telluride [16]. Bismuth antimony telluride is a p-type thermoelectric material, whereas bismuth selenium telluride is a n-type thermoelectric. These materials have great potential in heat recovery energy harvesting and can be used as refrigerating modules when used in segmented or cascaded power generation devices. 


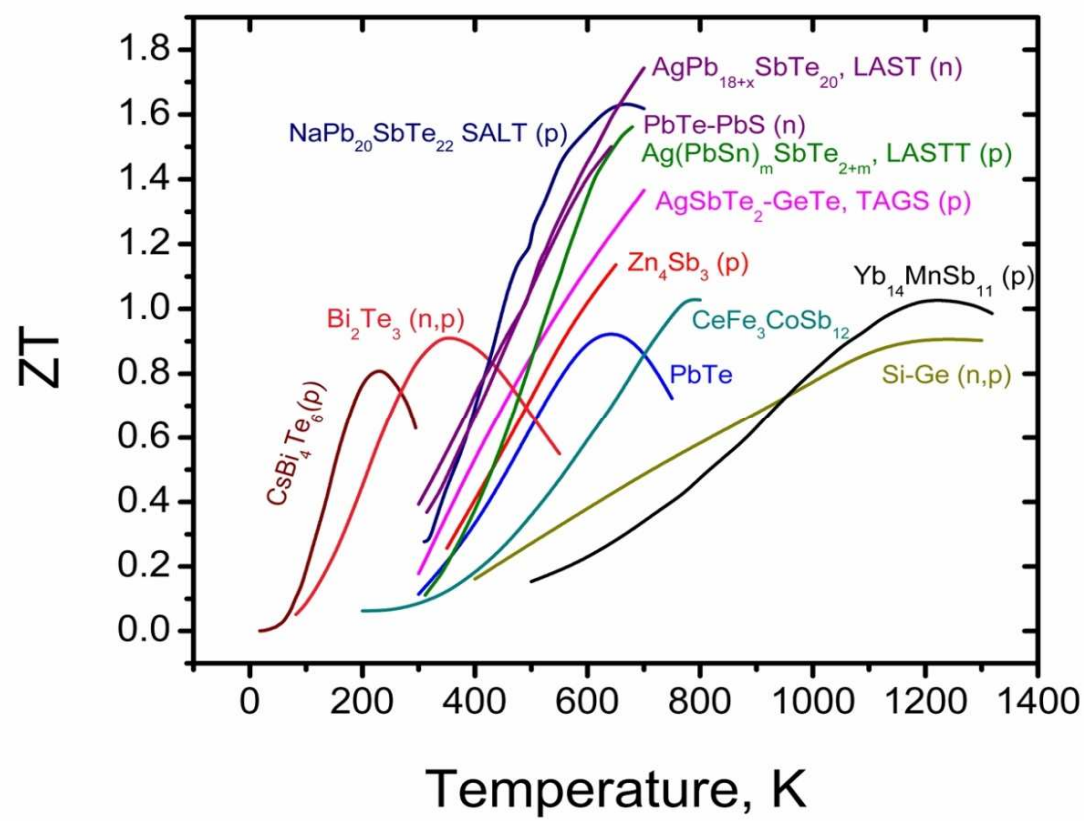

Figure 9: Figure of merit versus temperature for different thermoelectric materials[17]

Other parameters considered while selecting thermoelectric materials are carrier concentration. The range of carrier concentration for semiconductor materials typically varies in the range of $10^{18}$ to $10^{20} \mathrm{~cm}^{-3}$. Thermoelectric materials are heavily doped materials [7]. Materials with carrier concentrations outside the above mentioned range are either insulators or metals, both of which are poor for thermoelectric applications. Thermal conductivity of the material should be low as possible, since figure of merit (ZT) is inversely proportional to thermal conductivity.

\subsection{Review of Literature}

\subsubsection{Composites and thermoelectric materials}

Even though composites have been around for about a century, most research and developments were concentrated in areas of employing them as structural materials [18]. Composites are used in a wide range of fields ranging from aerospace industry to sporting industries. They are used in space shuttle tanks and also used to make golf putters. Combining composites with thermoelectric 
capabilities is relatively a novel approach, with limited advances. Previous studies into the thermoelectric composites have been done, in which the composite reengineering was done either by changing the matrix phase material or tailoring the interlaminar filler materials. Incorporating thermoelectric properties to composites was done by Chung et al. [19]. Processing, production scale-up difficulty, low ZT values, inadequate mechanical performance, and implementation difficulty where some of the problems associated with reengineering the composite for thermoelectric applications [19].

Though Seebeck effect was discovered by Seebeck in 1821[9], best figure of merit of these materials remained 1 for an extended period of time $[16,20]$. For efficient thermoelectric systems, either new material has to be synthesized or enhance the existing processes and materials.

Representative of the new material approach, materials like $\mathrm{CoSb}_{3}$ which reached a maximum ZT of 1.4 at $700 \mathrm{C}$ has been reported [21]. Following the second approach, improved thermoelectric transport properties were achieved by nano structuring [15, 22-24]. The maximum ZT in this field reported was employing the concept of super lattice [23, 24]. Over the last decade, several groups have reported enhanced ZT in super lattices such as $\mathrm{Bi}_{2} \mathrm{Te}_{3} / \mathrm{Sb}_{2} \mathrm{Te}_{3}$ and $\mathrm{PbSe}_{0.98} \mathrm{Te}_{0.02} / \mathrm{PbTe}$ [24]. New bulk materials like silver antimony lead telluride (LAST) and alloys [25] including Skutterudites [26], Clathrates [27], Zintl [28] phases have also been studied and improved.

The best materials to use for near room temperature application $\left(20{ }^{\circ} \mathrm{C}-250{ }^{\circ} \mathrm{C}\right)$ for power generation are bismuth antimony telluride and bismuth selenium telluride [16]. Other materials like Geranium, indium antimonite, Indium Arsenide, mercury telluride, mercury sellenide have been studied for their potential application at room temperature, but they all proved to be inferior to bismuth antimony telluride and bismuth selenium telluride.

Higher ZT were achieved through reduction in thermal conductivity, because of smaller grain size realized through nanostructuring [29]. Extensive experimental research and analysis of nano structured materials has given rise to a ZT of 1.45 at $100^{\circ} \mathrm{C}$ for p-type bismuth antimony telluride 
[30] and ZT 1.04 at $125^{\circ} \mathrm{C}$ n-type bismuth selenium telluride [31]. This enhanced ZT is achieved through ball milling and hot pressing nanoparticle alloys into bulk ingots. Enhancement in ZT using ball milling and hot pressing composite techniques has been reported by Mehdizadeh et. al.[32] for nanostructured composites of p-type bismuth antimony telluride. The reported ZT was 1.57 at $104.5 \mathrm{C}, 50 \%$ improvement over that of the conventional BiSbTe ingot. Xie et al. reported $\mathrm{ZT}=1.56$ at $27 \mathrm{C}$ using melt spinning method [33]. Fan et al also reported $\mathrm{ZT}=1.8$ at $43 \mathrm{C}$ using a combination of melting spinning and solid state reaction [34]. Zhao et. al has reported improvement in the $\mathrm{ZT}$ value by the addition of $\mathrm{Bi}_{2} \mathrm{Te}_{3}$ nanotubes [35].

Composite reengineering leads to many disadvantages, however the approach followed here is unique and more industrial oriented and guarantee the maximum thermoelectric power that can be extracted because of the temperature difference. Moreover, mechanical strength will not be compromised because of the incorporation of these thermoelectric devices on top of the composite rather than the interlaminar approach employed in previous works. The method of ball milling and hot pressing is followed here.

\subsubsection{Metal Contacts for thermoelectric materials}

In order to make thermoelectric devices, p-type and n-type materials have to joined electrically in series and parallel thermally [36]. An array of inter joining thermoelectric p-type and n-type materials obtain the maximum output. Joining of the p-type and n-type material are done by using electrical contacts/ ohmic contacts. The efficiency and reliability of thermoelectric device depends upon the metal contacts used. Moreover, the contact surface area between the thermoelectric material and ohmic contact is also crucial. Improper contact can lead to resistance flow of carriers, by reduction in the surface area between thermoelectric and contact. These contact materials, must be able to operate efficiently in the range of operation of the thermoelectric material too. 
The most commonly used material for electrical contact is copper, since it has very good electrical resistance of $1.68 \times 10^{-8} \mathrm{Ohm}$ meter at normal room temperature condition of $20^{\circ} \mathrm{C}$ [37]. This excellent feature has attracted much thermoelectric research along this line, on the application of copper in thermoelectric devices. However, even at room temperature copper diffuses into bismuth telluride [38] . Copper exhibits donor activity in bismuth telluride $\left(\mathrm{Bi}_{2} \mathrm{Te}_{3}\right)$ and is known for its high diffusivity in bismuth telluride and the formation of copper telluride $\left(\mathrm{Cu}_{2} \mathrm{Te}\right)$ [39]. Diffusion layer of $\mathrm{Cu}_{2} \mathrm{Te}$ was not derisible, since it reduces the thermoelectric performance of the thermoelectric element. Copper electrodes are being widely used today for thermoelectric module applications. Key to using copper as conducting metal is to prevent formation of diffusion layer, by introduction of a barrier layer of sufficient thickness.

Suitable materials if used at the interface will prevent diffusion. These materials are known as diffusion barrier layers. Much research have gone into finding a suitable barrier layer for preventing diffusion. The prevention of diffusion layer has to be addressed direly in thin films technology since, dealing with materials of micron and sub-micron level thickness. Traditionally, nickel has been employed as the barrier material of choice for bulk thermoelectrics (or Peltier) cooling devices [20]. Nickel is a diffusion barrier for preventing copper into the thermoelectric element. However, nickel by itself diffuses into the thermoelectric element [40]. Alternate, metal like Tin being used instead of copper also resulted in the diffusion of Tin into the thermoelectric[41]. Employing nickel as barrier layer resulted in no diffusion of copper into the thermoelectric. Moreover, nickel also acted as a good barrier in the case of Tin bismuth soldering alloy [40]. Nickel diffusion into the thermoelectric element has been observed, but this effect is negligible for both p-type and n-type bismuth telluride alloys, when dealing with bulk materials [40]. Same results are supported by the study conducted about the diffusion of nickel into the thermoelectric interface and confirm the formation of nickel telluride $\left(\mathrm{NiTe}_{2}\right)$ at the interface of bulk bismuth selenium telluride and nickel [42]. However, nickel prevents the diffusion of copper 
and tin into the thermoelectric elements very efficiently. Solder material like tin forms nickel-tin alloy at the interface. Other studies conducted on thin film thermoelectric materials also suggests that cobalt metal can also acts as good metal contact, because of its better adhesion and lesser diffusivity into the thermoelectric materials [42]. Copper diffusion into bismuth telluride $\left(\mathrm{Bi}_{2} \mathrm{Te}_{3}\right)$ was observed and a layer of significant thickness of diffusion accompanied by color change was observed at the interface. Contact metals on thermoelectric elements have been done employing MEMS techniques [43], some of which are Metalorganic Chemical Vapour Deposition (MOCVD), sputtering, Physical Vapour Deposition (PVD), electrochemical plating, soldering and laser welding [44]. 


\section{CHAPTER II}

\section{CHARACTERIZATION AND MEASUREMENT METHODS}

\subsection{Introduction}

The fundamental transport properties of thermoelectric materials are used to characterize the performance of thermoelectric elements. Thermoelectric materials are best described quantitatively by the figure of merit (ZT). As mentioned in the previous chapter, the figure of merit depends upon three transport parameters, electrical conductivity, Seebeck coefficient and thermal conductivity. The temperature dependence of these parameters measures the performance of the thermoelectric elements. All these properties are measured using commercially available techniques.

Measuring corresponding voltage change over temperature and current applied, Seebeck coefficient and electrical conductivity can be measured. Ulvac "ZEM-3" was used for measurement of Seebeck coefficient and electrical conductivity and laser flash for thermal conductivity. ZT value is obtained from the measurement of these parameters.

Electrical resistivity of thin film was measured by using a four point probe. It is a very precise method of measuring the resistivity of thin films. Measurements methods are briefly explained in following sections.

An X-Ray Diffractometer was used to ensure and confirm that the desired material phase and alloy was achieved. A Hitachi S-4800, Scanning Electron Microscope (SEM) was used to study 
the grain formation. Energy-Dispersive X-ray Spectroscopy (EDS) data was collected using IXRF EDS system to obtain atomic concentration of individual elements. These machines were also used to evaluate the grain size and alignment. All the experiments carried out are reproducible. All the measurements from the equipments mentioned below are error free and fully reproducible under similar conditions.

\subsection{Characterization Techniques}

\subsubsection{Scanning Electron Microscope}

SEM was used for characterization purposes. It was used to study the grain size and formation in powder, thin film and bulk samples. EDS was used to find out the stoichiometry of the powder, thin films and bulk samples. The average atomic concentrations were obtained.

\subsubsection{X- Ray Diffraction}

$\mathrm{X}$-Ray Diffraction (XRD) is a versatile and non-destructive technique that reveals detailed information about chemical composition, physical properties and crystallographic structure of materials[45]. Bruker AXS, Discover D8 X-ray Diffractometer (XRD), was used for all the XRD measurements.

X-rays waves having very small wavelengths, impinged on the crystal lattice leads to scattering. Scattered waves produce a unique pattern corresponding to the material, which can be analyzed to study the crystal pattern and orientation. Each material has its own unique diffraction pattern. Comparative analysis will give us an idea about the chemical composition of the unknown material. The crystal size was approximated from the plots and software analysis. 


\subsection{Measurement Techniques}

\subsubsection{Electrical conductivity and Seebeck coefficient measurement}

A quick, dependable and commercial method of determining the electrical and conductivity and Seebeck co-efficient was obtained using the ZEM-3 from Ulvac Inc. Figure 10 shows a schematic of the method used for the electrical conductivity measurement.

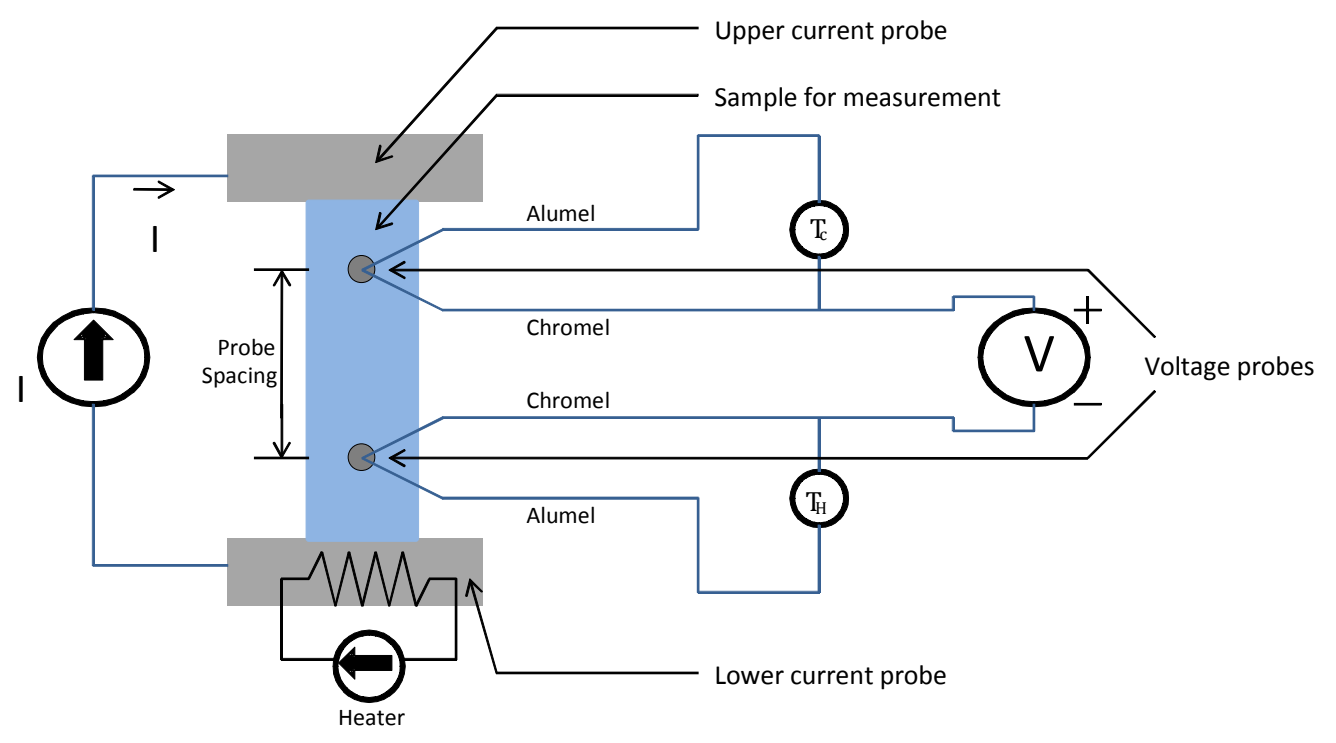

Figure 10: Schematic of Seebeck-electrical properties measurement system

Electrical conductivity was measured using a four point probe method, therefore voltage drop could be neglected. The sample to be measured was held in between two jaws, through which current was passed through to the sample. This accounts for two of the four probes. An electrical heater resistance is provided at the lower jaw, which was responsible for creating the temperature gradient along the sample length. Two probes are used to measure the voltage and temperature drop across the sample, these probes are spring loaded to ensure that there is good and direct contact between probes and sample. Constant spacing is assumed between these voltage probes. This spacing is accounted for in the calculation of the electrical conductivity and Seebeck coefficient calculations. This arrangement is surrounded by a nickel radiation shield to ensure 
isothermal environment. The setup is enclosed in an infrared furnace with helium atmosphere, to prevent oxidation of the sample at elevated temperature measurements.

Sufficient pressure should be applied in between the samples and all the contact surfaces to reduce the contact resistance. Voltage losses over these contact yield incorrect voltage values. To approximate that, the heat and current flow is in one dimension, the distance between the probes and end plates should be 1.5 times larger than the samples characteristic length. The samples for the ZEM measurements are all rectangular in shape having varying dimensions. For a typical case the dimensions of the sample were $2 \mathrm{X} 2 \mathrm{X} 12 \mathrm{~mm}$. Figure $31(\mathrm{~b})$, shows the picture of sample cut for ZEM measurement.

A pair of chromel-alumel (type-K) thermocouples using two channels of Keithley 2010 digital multimeter was used for the temperature measurements for the top and bottom probe temperatures. Chromel leads were used to measure voltage differential between probes for the Seebeck and resistivity measurements. AC supply was used to over the effect of voltage produced by Peltier effect. For accurate values the measurement is taken twice, changing the polarity of the current and also making the current duration short. The two combine to give the resistance of the sample. Figure 11(a), shows the picture of ZEM equipment used for measurement, (b) sample loaded on the system in between the probe space. From V-I curve, resistance and consequently resistivity can be determined at various temperatures.

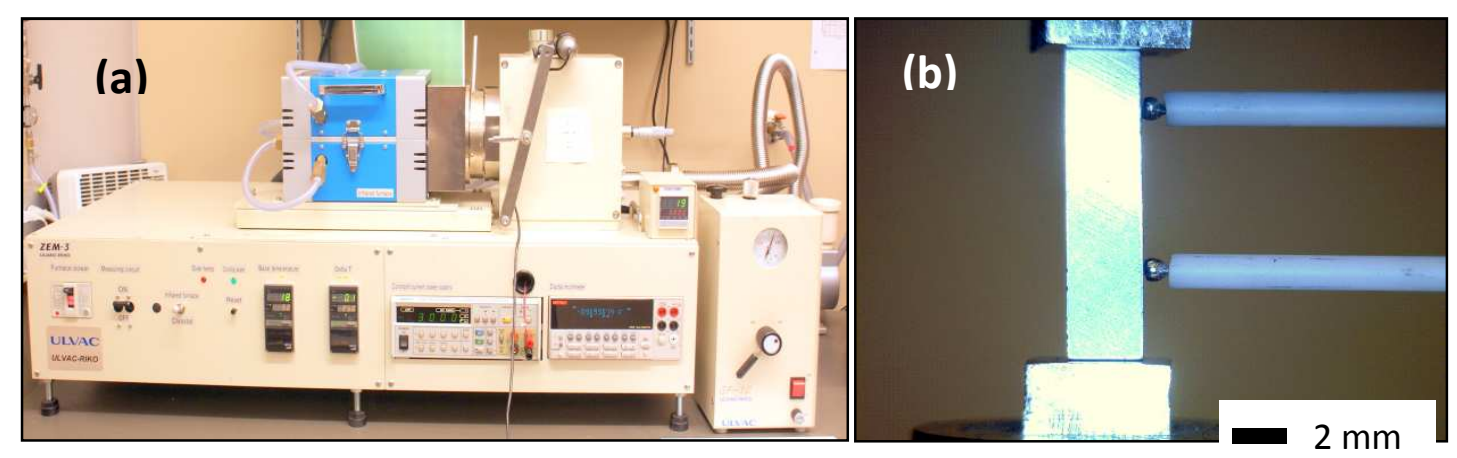

Figure 11: (a) Ulvac ZEM (b) Sample mounted on ZEM 
For Seebeck co-efficient measurement, voltage differences of the sample over different temperatures are used to calculate. No current flows through the sample, the only source of EMF is from the Seebeck effect in the sample and the probe leads. A schematic for Seebeck coefficient measurement is given in Figure 12.

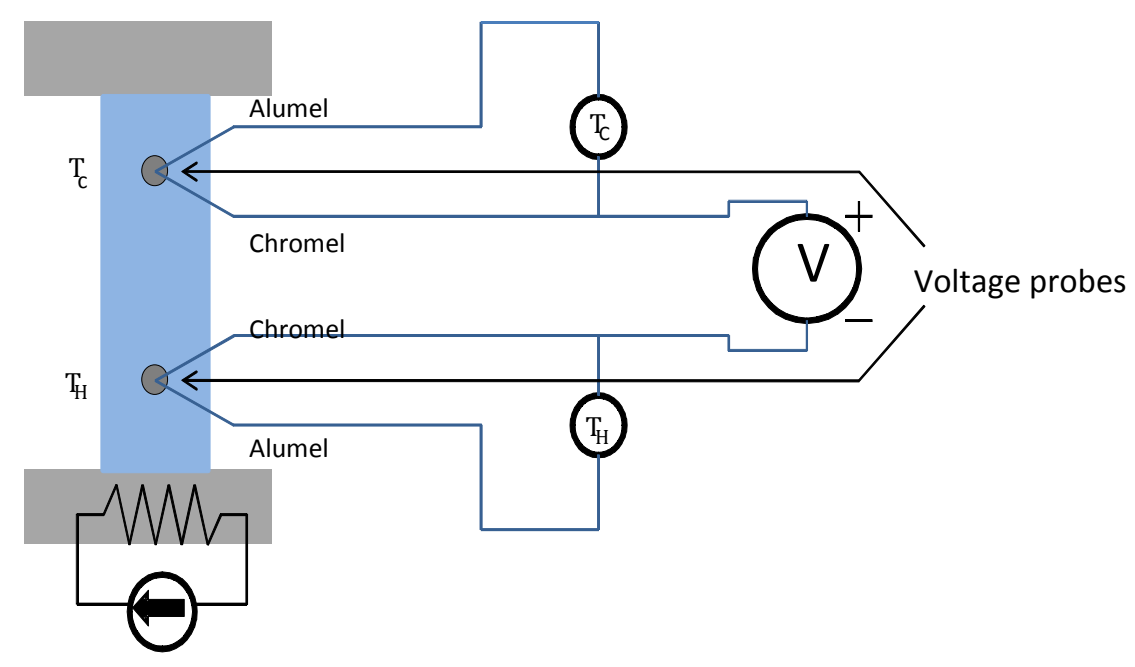

Figure 12: Seebeck coefficient measurement setup, temperature difference exists between the upper and lower probes

The following expression is used for computing the Seebeck coefficient, given equation 1.3.

$$
S=-\frac{d V}{d T}
$$

Seebeck value is calculated from the slope of $\Delta \mathrm{V}$ vs. $\Delta \mathrm{T}$ plot. Seebeck at different temperature intervals were done to measure the highest Seebeck coefficient.

\subsubsection{Thermal conductivity measurements}

Thermal conductivity measurements were done using Laser flash equipment by Netzsch L.L.C. Figure 13 shows the picture of the laser flash used for the thermal conductivity measurements. 


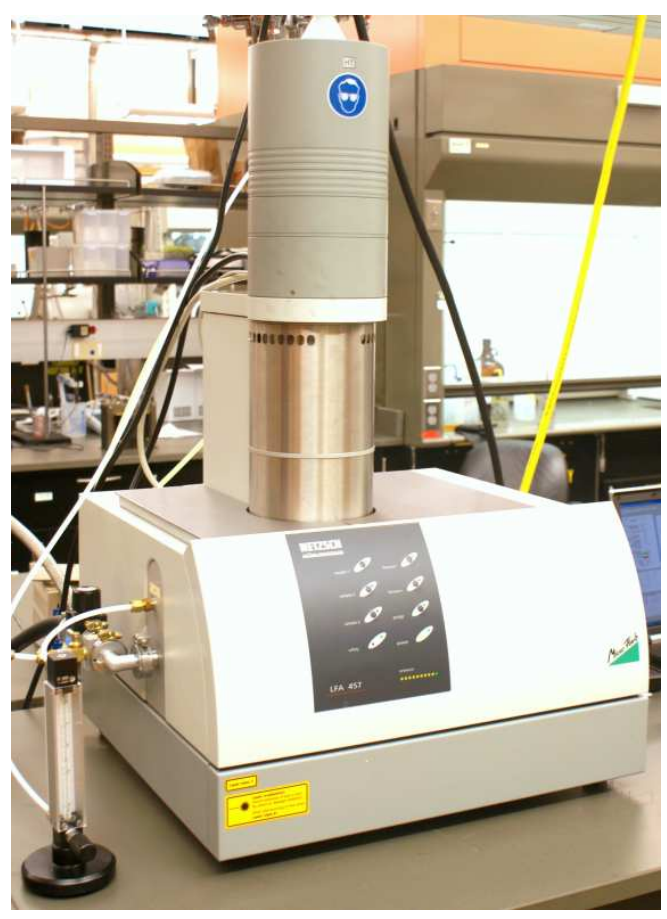

Figure 13: Picture of Netzsch laser flash used for thermal conductivity measurements

A disc of about $12.7 \mathrm{~mm}$ diameter and $2 \mathrm{~mm}$ thickness is used as sample for thermal conductivity measurements using a laser flash. Figure 31(b), shows the picture of a laser flash sample diced and polished and ready for measurement. There should be sufficient thickness for the sample to ensure accurate thermal conductivity measurements.

Schematic of laser flash working is shown in Figure 14. Laser flash basically contains a tube in which the sample is loaded. This tube in the laser flash equipment is the furnace and can be maintained at different temperatures for the measurement at different steps. A laser beam is used to hit the sample on one surface and the infra-red radiation transmitted through the sample is collected on the Indium-Tin Infrared Detector after passing through the lens. A laser flash is used to overcome the problem of physical contact thus eliminate thermal contact resistance. Moreover, precise values of thermal conductivity are almost guaranteed, by making the measurements for short time. 


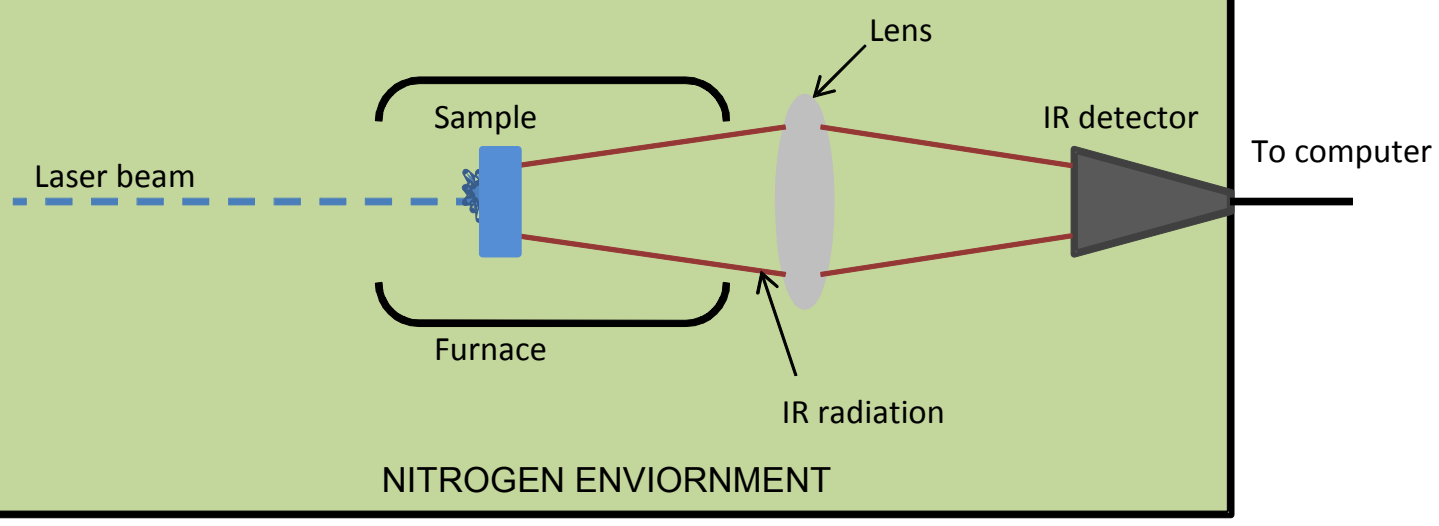

Figure 14: Schematic of laser flash working principle for thermal conductivity

measurement

\subsubsection{Resistivity measurement using four point probe method for thin films}

Since, electrical conductivity is inversely proportional to resistivity, measuring electrical resistivity will provide information about the conductivity of materials. Resistivity is measured using the 4 point probe method.

The four point probe method is widely used for measuring the resistivity of a semiconductor material to overcome voltage drop due to contact resistance. It can be used for the measurement propose of both bulk and thin film materials.

As the name suggests, there are four probes, two current probes and 2 voltage probes. Figure 15 shows (a) schematic and (b) picture of the four probe used for resistivity measurement. The probes are equally spaced tungsten metal tips with finite radius. Outer probes are used to send the current and the inner probes are used to measure the voltage drop developed over the sample. Typical spacing between the probes is $\sim 1 \mathrm{~mm}$. 

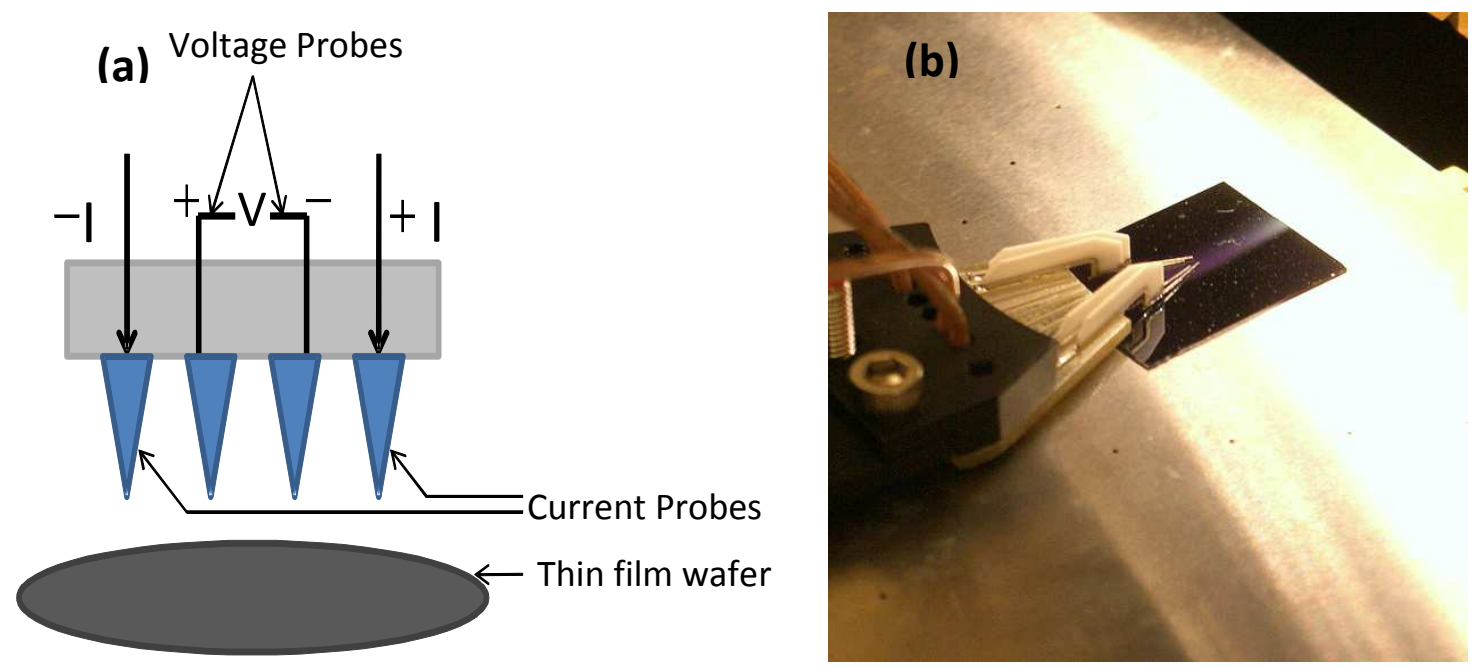

Figure 15: (a) Schematic, (b) picture of 4-point probe configuration

Theory behind measurement of the 4 point probe is stated below.

The differential resistance equation is given by equation 1.4

$$
\Delta \mathrm{R}=\rho\left(\frac{\mathrm{dx}}{\mathrm{A}}\right)
$$

Where $\Delta R$ - change in resistance

$$
\rho \text { - Density }
$$

A - Area under consideration

$\mathrm{x}$ - Distance between the probes

Considering rings of current emanating from the outer probes, the area expression becomes $\mathrm{A}=$ $2 \pi \mathrm{xt}$, where " $\mathrm{t}$ " is the thickness of the thin films. Integrating resistance over the distance in between the inner probes, we get the following equation 2.3.

$$
\mathrm{R}=\frac{\rho}{2 \pi \mathrm{t}}(\ln 2)
$$

Now replacing resistance $(\mathrm{R})=\mathrm{VI}$ in equation 2.3

$$
\rho=\frac{\pi \mathrm{t}}{\ln 2}\left(\frac{\mathrm{V}}{\mathrm{I}}\right)
$$


Thus sheet resistivity $\mathrm{R}_{\mathrm{S}}$ can be further written in equation 2.5

$$
\mathrm{R}_{\mathrm{s}}=\mathrm{k}\left(\frac{\mathrm{V}}{\mathrm{I}}\right)
$$

$$
\begin{aligned}
\text { Where } \mathrm{R}_{\mathrm{s}} & =\left(\frac{\rho}{\mathrm{t}}\right) \\
k & =\left(\frac{\pi \mathrm{t}}{\ln 2}\right)=4.53
\end{aligned}
$$

Substituting the value of $k$ in equation 2.5,

$$
\mathrm{R}_{\mathrm{s}}=4.53\left(\frac{\mathrm{V}}{\mathrm{I}}\right)
$$

For ease of measurement, we can tune the current to the value of $0.453 \mathrm{~mA}$ and resistance will be the value of voltage displayed.

The experimental setup used for measurement of the thin film resistance is shown in Figure 16.

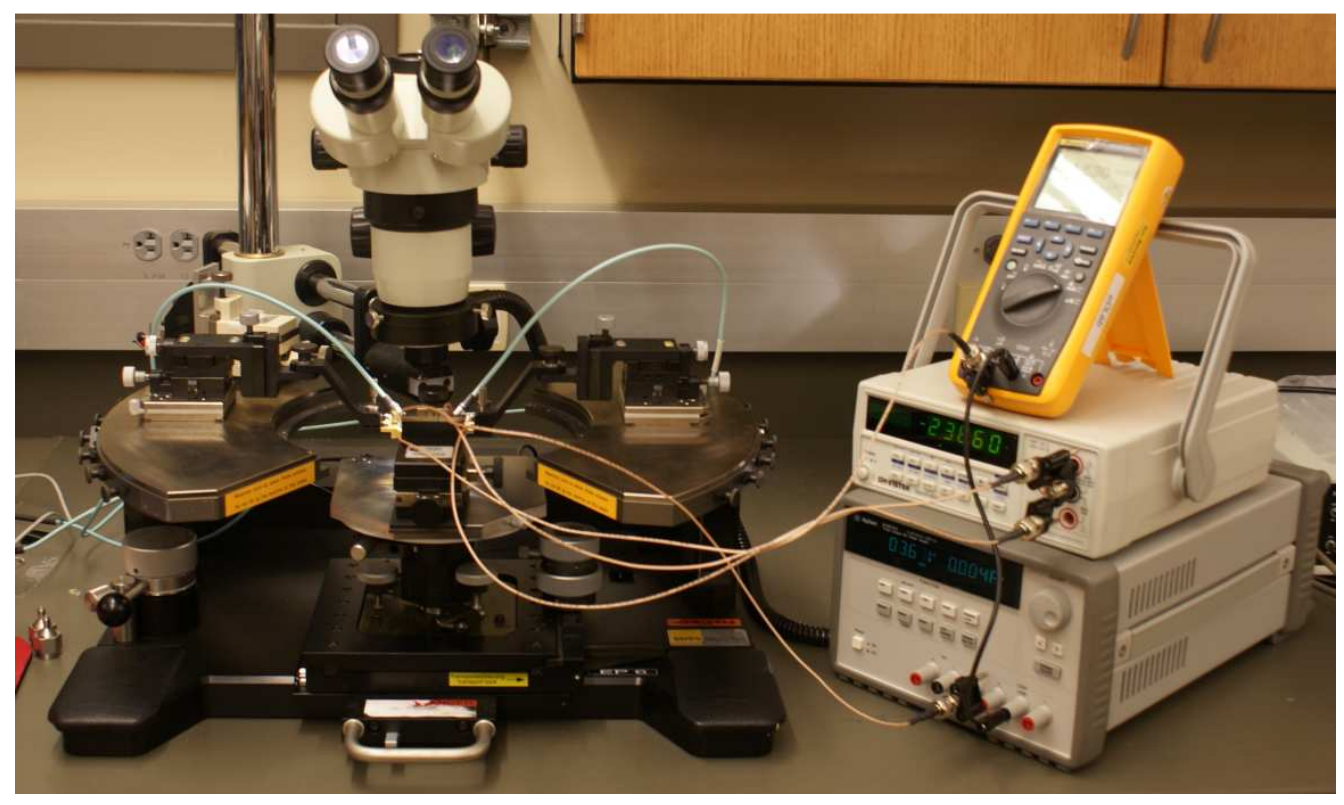

Figure 16: Four probe measurement setup for thin film resistance measurement 


\section{CHAPTER III}

\section{EXPERIMENTATION}

\subsection{Polymer Thermoelectrics}

The initial step of realizing the multi-functional embedded thermoelectric structural composites was to make thermoelectric devices, to be incorporated on composites surfaces. Key parameters for consideration during construction of thermoelectric composite were quick manufacturing and economic viability. It was reckoned that a polymer film based thermoelectric approach would be better since, integrating the polymer film onto composite surface could be easily done. The polymer polyvinylpyrrolidone (PVP) was selected, since it would be compatible with most resin system of the composites. The concept was to blend thermoelectric powders into polymer, based on the successful previous work carried out to blend carbon nano tubes into PVP polymer [45]. These polymer experiments are briefed in Appendix A. They were discontinued, as they yielded poor preliminary results and needed further investigation.

To fabricate the thermoelectric device, two approaches were followed here, thin film approach and bulk material approach.

\subsection{Thermoelectric device}

A typical thermoelectric device as mentioned in the previous chapters is a combination of both ptype and n-type materials. Schematic of a single p-n junction is shown in Figure 17. 


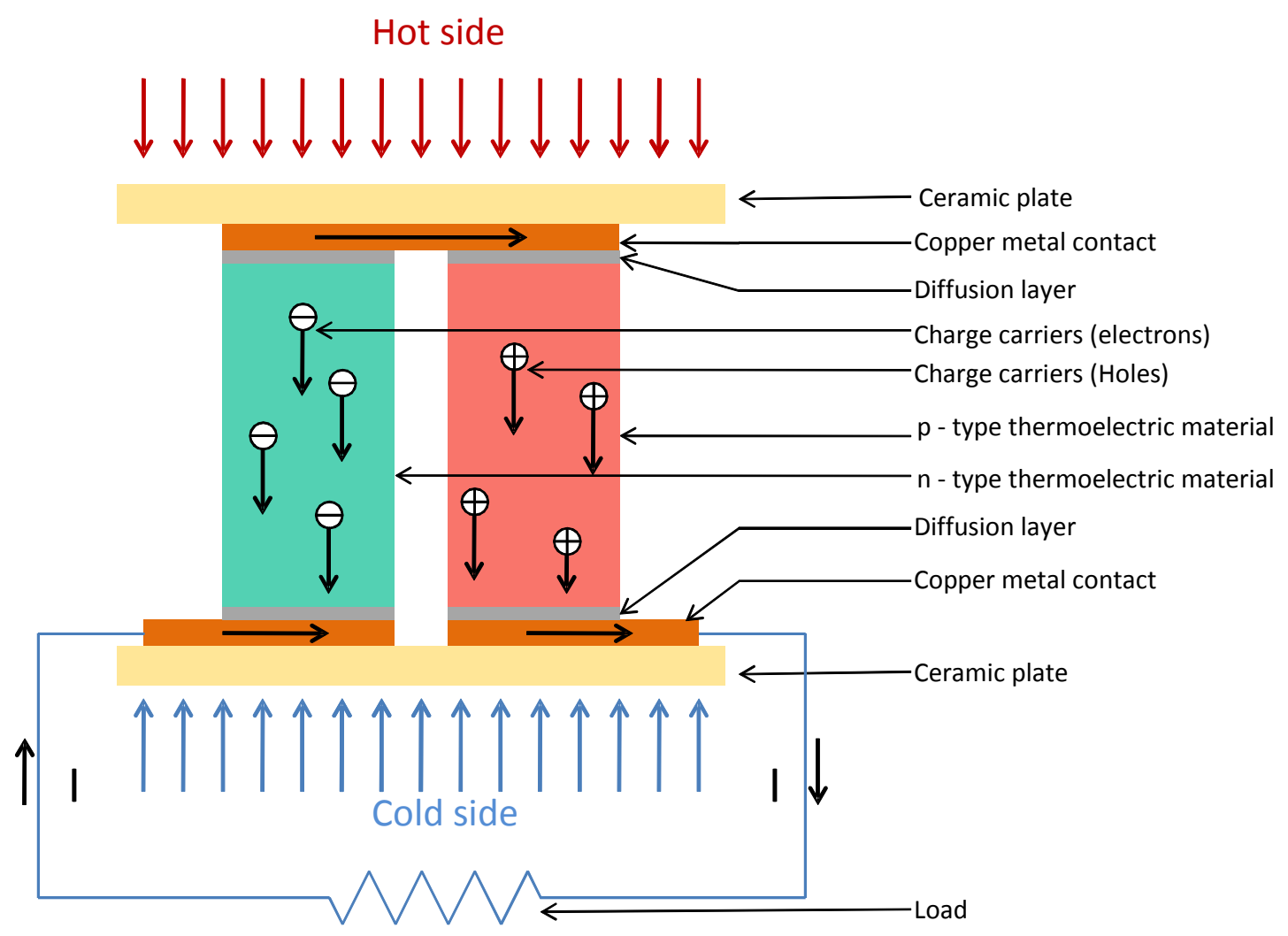

Figure 17: Schematic of thermoelectric $\mathbf{p}$ - $\mathbf{n}$ junction, with metal contacts and ceramic

$$
\text { plates }
$$

The majority charge carriers in p-type material are holes, whereas in n-type they are electrons. As mentioned previously in chapter 1 , due to temperature difference, charge carriers at the hot side will be at a greater potential than the cold side carriers. Carriers diffuse from an area of higher potential to that of lower potential and will lead to a built of charge potential, resulting in a voltage. The electrons move opposite to that of the current and holes move in the direction of the current. The device is connected to a load, which can be a motor or bulb. The schematic represents a single $\mathrm{p}-\mathrm{n}$ thermoelectric device. Many of them connected in arrays will lead to a thermoelectric module. In order to achieve this device, $\mathrm{p}$-type and n-type thermoelectric materials with suitable diffusion barrier layers and metal contacts have to be fabricated individually and mated together to form the final thermoelectric device. 


\subsection{Thin film thermoelectrics}

\subsubsection{Introduction}

The ranges of thickness attributed to thin films are of order of nanometers to micron. Thermoelectric materials are deposited on a substrate by different techniques. With the onset of the nano-technology, more hike has been experienced in the thin film industry. The whole engineering of MEMS field revolves around thin film approach.

MEMS (Micro Electro Mechanical Systems) are a field of science, where nanoscaled devices are created using different processing techniques. The unique approach in this field is the aim of miniaturization of the system. Usual MEMS devices vary from a scale of 0.2 micron to almost as big as $1 \mathrm{~mm}$. Most MEMS devices are found in computers and electronic devices.

There are many methods of deposition of thin film on a substrate Plasma Assisted Chemical Vapor Deposition (PECVD), Low Pressure Chemical Vapour Deposition (LPCVD), Metalorganic Chemical Vapour Deposition (MOCVD), Physical Vapour Deposition (PVD), Sputtering, Pulsed laser deposition, Molecular Beam Epitaxy (MBE). Sputtering process was used because of the availability of the equipment, compared to all the other methods.

\subsubsection{Objective}

Aim of this approach was to make thermoelectric thin film devices as shown in Figure 17. The initial step was to deposit thin film thermoelectric materials and characterize the optimum depositing conditions. 


\subsubsection{Equipment}

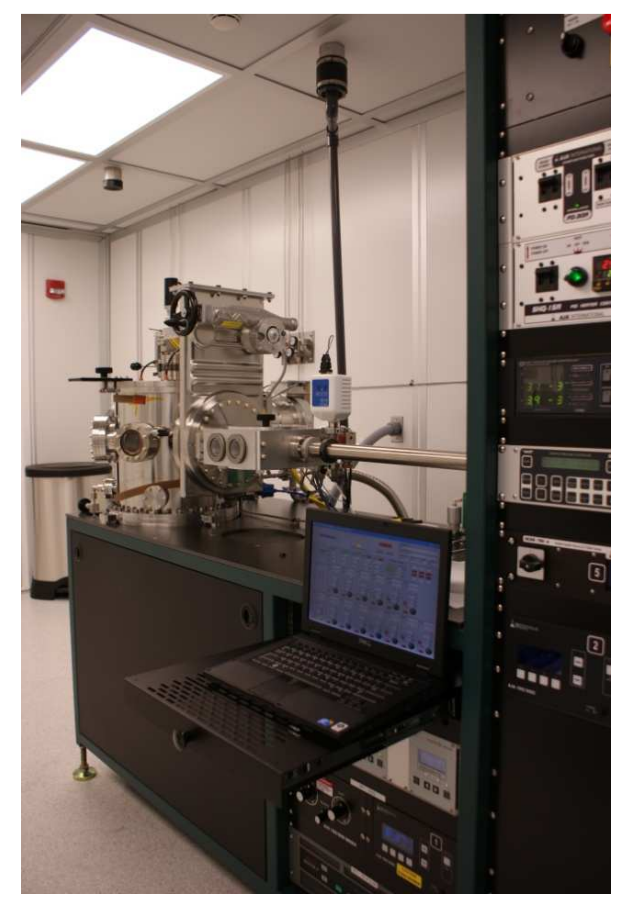

Figure 18 : AJA Orion Sputtering Equipment used for thin film depositions

AJA Orion 8 sputtering machine was used for thermoelectric thin film deposition. Figure 18 : AJA Orion Sputtering Equipment used for thin film depositionsshows sputtering equipment used for this purpose. The sputtering machine mechanism is elucidated briefly. It can be used for deposition of both insulator and metallic materials. The process is slow compared to other thin film deposition techniques like PECVD and evaporation.

Schematic cross section of a sputtering equipment setup is shown in Figure 19. A sputtering machine essentially contains a highly vacuumed chamber which houses both the thin film source (target) and the substrate. The targets are placed inside an enclosure called "GUN", which also contains both the cathode and anode. Extremely high vacuum pressure of $10^{-8}$ torr is maintained inside the sputtering chamber to avoid unwanted air molecules and water vapour, thus increasing the mean flow path of the ions, electrons or target atoms. Argon gas molecules dissociate into argon ions and electrons. This ionization of gases takes place when plasma is created. Argon gas 
is flowed under control into the chamber when the sputtering process is carried out. Variable potential difference can be maintained between two electrodes, cathode and anode by electrical supply to sustain the plasma ionization process. Sputtering target is housed on the cathode inside the gun. The ionized argon ions are attracted by the negatively charged cathode. After the formation of the ion, the heavy ions are accelerated towards the target employing the potential difference in between the electrodes. Heavy and high energy argon ion impinges on the target surface and target material atoms are released, which will deposit itself on the substrate. The energy of the incoming argon ion will be more than the binding energy between the target atoms, thus releases a target atom from the surface. This collision and release of electrons and atoms generate enormous amount of heat, which has to be cooled quickly. Electrons released create more and more ionized argon ions, making the reaction sustainable, this process will continue as long as there is a steady supply of argon and potential difference is maintained between electrodes.

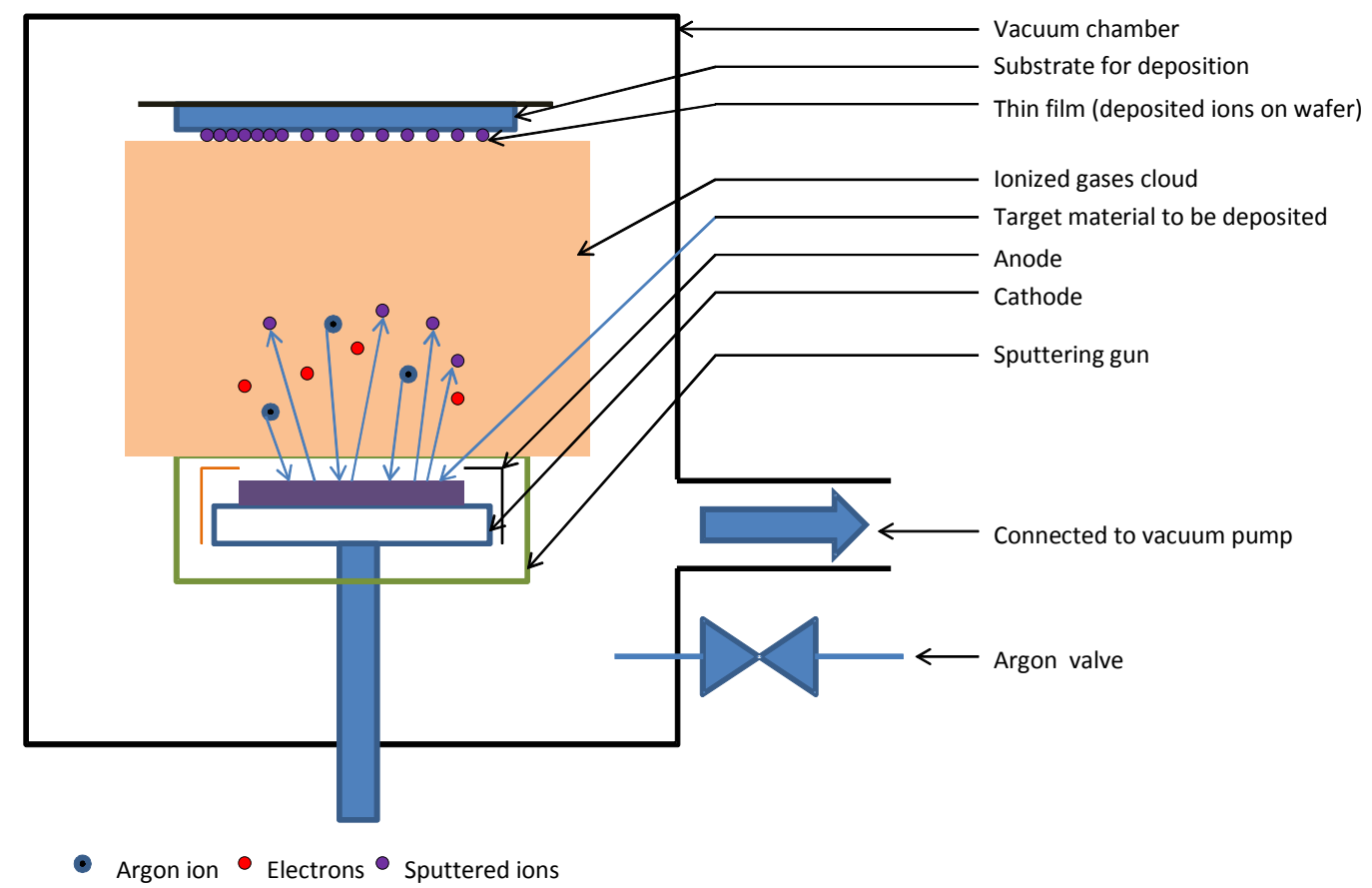

Figure 19 : Schematic of sputtering chamber 
There are many parameters that can control the deposition process and composition of the thin film in the sputtering process. Figure 20, shows all these parameters, in a pictorial representation.

1) Chamber pressure - Less pressure inside the chamber, implies higher vacuum and more availability of argon ions for sputtering process relatively, thus increasing the net mean flow path. Therefore low pressure is derisible inside the chamber for sputtering.

2) Argon gas flow - Increase in argon gas flow gives rise to more argon atoms available for sputtering, but also gives rise to lower amount of ionized argon atom. Less argon flow gives rise to lack of argon atom and leads to inefficient sputtering process. Argon gas flow also ensures a clean environment.

3) Target power - The power or potential is applied to the electrodes, greater power ensures high energy ions for the bombardment process of target atoms. Power used can be primarily either DC (Direct Current) power or RF(Radio Frequency) power. DC power is appropriate for metal targets while RF power is suitable for both insulators and metal targets. The deposition rate is drastically reduced if RF power is used for metals. This is the power which directly affects the acceleration of the argon ions and thus the ionization rate. More power or potential applied between the electrodes, more kinetic energy and greater sputtering rates. The heat produced on target surface will also increase simultaneously as power is increased.

4) RF Bias Power - The substrate holder can be supplied with power to assist incoming target atoms with more energy and this leads to increased density for the thin film. Excessive power will lead to back-scattering and loss in deposition rate. RF power supply is connected to the substrate for bias power.

5) Substrate temperature - Supplying substrate temperature gives the incoming electron more energy and gives it ample time to align itself in an orderly manner on the substrate. 
6) Working Distance - The distance of the substrate from the target material also affects the properties of the film. Greater distance travelled by the atoms, reduces their energy and deposition rate will be low.

7) Rotation Speed - The substrate on which the thin film is deposited is constantly rotated so that, there is uniformity of the film on the substrate. A nominal speed is recommended.

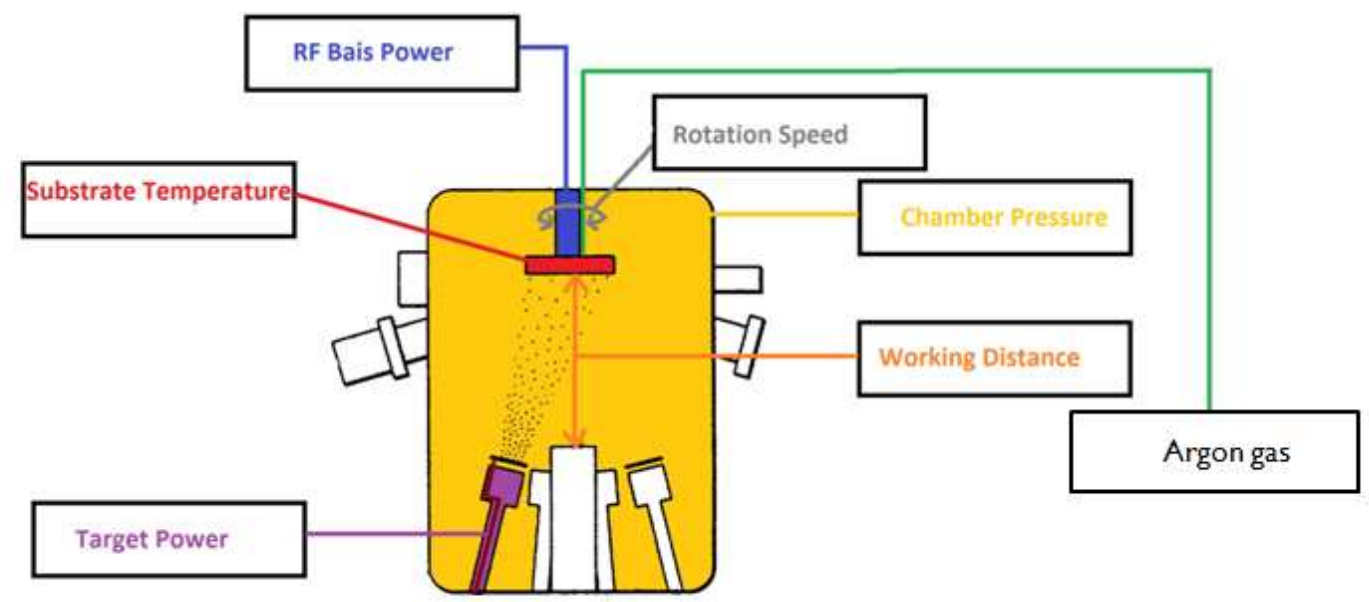

Figure 20 : Sputtering Parameters

\subsubsection{Thermoelectric thin film Experimentation}

In order to make the thermoelectric thin film, optimization of previously mentioned sputtering parameters had to be done. The final thin film obtained should possess high density, correct thermoelectric stoichiometric ratio and sufficient deposition rate etc. Individual targets for bismuth telluride and antimony telluride were prepared and sputtering was done for obtaining the final thin film of bismuth antimony telluride (ternary alloy).

\subsubsection{Method of preparation of Targets}

Target is the material bulk/source that has to be sputtered and deposited on the substrate. The target source had to be a 2 inch diameter disc of 0.250 inch thickness, to be compatible for loading into the sputtering machine gun. 
Alloyed powders were tried making into a single bulk target by cold pressing. The process of alloying is explained in the next chapter. Many attempts at cold pressing failed, as the powders, even though were compacted in a two inch diameter stainless steel die a maximum pressure of 11 tons, upon ejection from the die because of the vibration, the target cracked. Attempts to make sintered nanostructured target was also carried out. Sintering the alloy powders at high temperatures to make a bulk sample were carried out. Even though sintering was carried out, the target sample cracked upon ejection from the graphite die, because of its large diameter and brittle property of alloys of bismuth telluride. Spark plasma sintering was used for hot pressing the target powders, but because of large diameter of the sample, heat was not uniformly distributed across the whole sample. This method is further described in the next chapter.

A polycrystalline melt ingot of the alloy powder proved to be most efficient. Elemental powders of bismuth, antimony and tellurium were weighted and mixed manually inside a glove box in a argon atmosphere to prevent oxidation of the powders. These were then loaded into a graphite crucible and loaded into a quartz glass tube and vacuum sealed for melt forming the alloy. Induction furnace was employed for alloy formation. This ensures homogeneity and saved time compared to the mechanical alloying process. Temperature maintained inside the crucible during alloy formation was about $800^{\circ} \mathrm{C}$, which is above the melting temperature of bismuth telluride alloys. Melting point of bismuth telluride is $600^{\circ} \mathrm{C}$. As a result of the magnetic field created, the melt starts to churn inside the graphite crucible, ensuring uniform alloying. In an induction furnace ingot formation was done at argon inert atmosphere. Figure 21 (a), shows the schematic of the induction furnace setup and (b) shows the picture of the setup during experiment. Alumina blocks are provided above and below for support and heat trapping. Melting temperature of bismuth telluride alloys are at $600^{\circ} \mathrm{C}$. Quartz tube can withstand high temperatures of $1200^{\circ} \mathrm{C}$. 

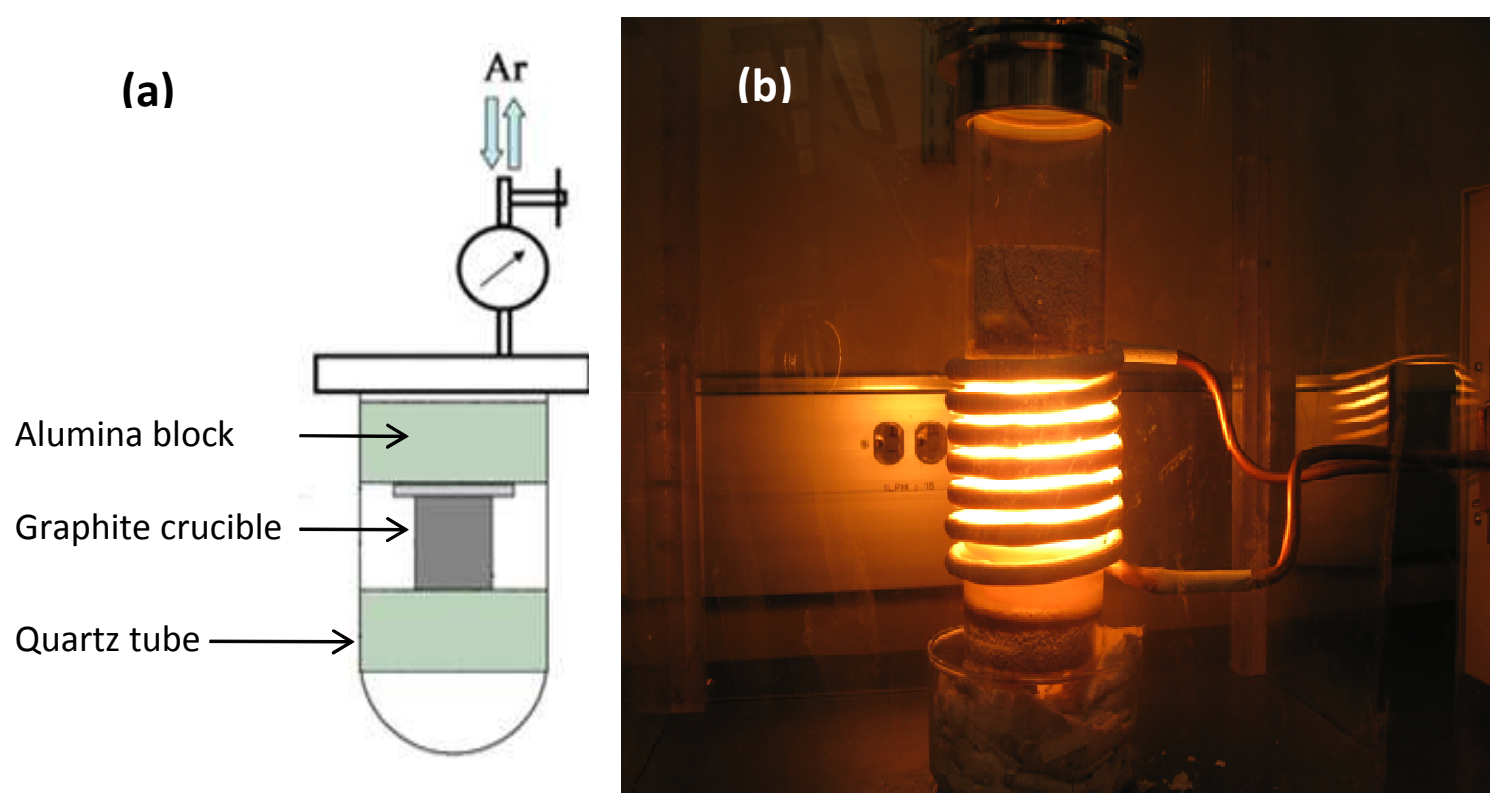

Figure 21 : (a) Induction furnace Schematic (b) Induction furnace experimental setup

A problem endured during the induction furnace melting process was, large amount of loss of tellurium from the mix. Tellurium has a very low vapor pressure and it evaporates out and leaving a tellurium deficient alloy. To compensate this loss, we add extra tellurium.

In a typical procedure, bismuth and tellurium powders of 200 mesh was weighted out in the atomic ratio of 34:66. The atomic stoichiometric ratio of bismuth to telluride is $40: 60$, following the chemical formula ( $\quad$ ). From previous experiments conducted, it was observed that, during induction furnace melting and sputtering, 3\% of tellurium was lost individually. In total, $6 \%$ of tellurium is lost in the deposited thin film. The end product of the atomic ratio (36:34) was assumed to give appropriate stoichiometric ratio of (40:60) to thermoelectric films. The current of the induction furnace is controlled to induce more eddy currents and thereby increase the temperature. The tube is vacuumed and argon purged several times before melt forming the alloy powder into ingot. Similarly, a target of antimony telluride was formed.

Ingot obtained does not possess a disc shape. As obtained ingot from the induction furnace is shown in Figure 28. The disc shape was obtained by melting the ingot in an appropriate shaped 
graphite die at just above the melting temperature of the alloy and holding it for an extended time. The melt acquires the shape of graphite crucible. The final target obtained was a cylindrical two inch diameter shaped target, which was easily removed from the graphite die. Figure 22 (a), shows a target formed in the above mentioned procedure.
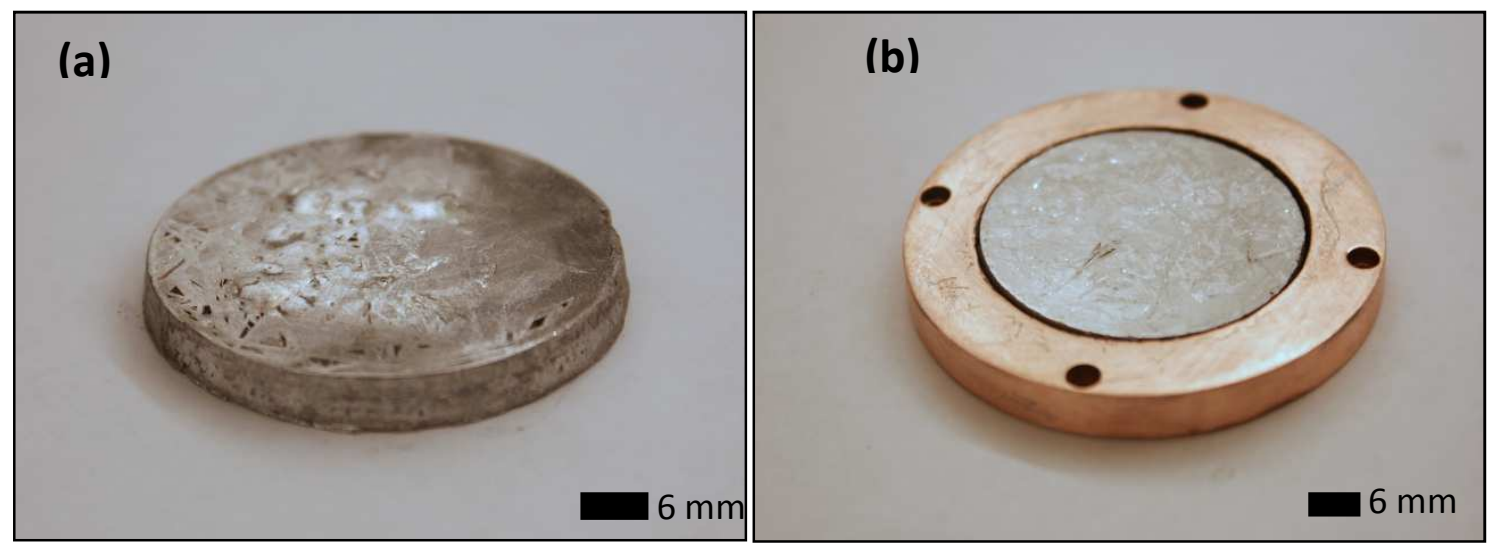

Figure 22 : (a) Picture of target after processing and shape formation, (b) Target bonded to copper cup for better performance

The target obtained was loaded on to the sputtering machine. Low thermal conduction, property of the thermoelectric material makes cooling the target inefficient. Many attempts at efficient cooling were tried, bottom surface of the target was polished to high finish and a copper mesh was used for effective heat dissipation, proved unfruitful. Low sputtering powers generated lesser heat but compromise had to be made on the deposition rates. To overcome the problem of heating up of the target and application of more power on the target, it was decided to house these targets in copper cups, picture shown in Figure 22 (b). The targets were bonded on to the copper cup with bismuth powder. Bismuth has a low melting point as compared to other elements and was compatible with the alloy target that was to be bonded.

Another method of efficient bonding thought was melting the alloy powders in the copper cup. This proved to be a disaster as extra tellurium in the alloy powder reacted with the copper and 
formed a stable phase of copper tellurium that was more stable than the bismuth antimony tellurium phase.

\subsubsection{Sputtering experiment}

Individual targets of bismuth telluride and antimony telluride were fabricated. Sputtering the two targets would have to be done in a alternating manner for the final thermoelectric thin film. Optimized condition for sputtering the individual compounds had to be found out individually. Bismuth telluride and antimony telluride target optimization was carried to certain extent, to obtain the highest overall efficiency, in terms of density, deposition rate and stoichiometric composition of the thin film.

The substrate used for all thin film depositions was silicon wafer. Silicon wafer were used for SEM and EDS purposes, in order to avoid charge up during SEM image procurement. However for sheet resistance measurements, silicon wafer with two micron thick Silicon-di-oxide was used. Silicon-di-oxide, an electrical insulator, was obtained by growing the oxide layer in a PECVD.

As a trial experiment, in order to study the behavior of the alloy, a single target of bismuth antimony telluride ternary alloy was prepared. Appendix B shows the change in parameters for different sputtering conditions for bismuth antimony telluride alloy. Since, this was the first time a target fabricated in the laboratory was used for sputtering process, many random experiments were conducted to know the efficient method of sputtering and compatibility with the equipment compared to commercially available targets used for sputtering. The results of these trail experiments are erroneous. These experiments helped to study the behavior of these materials when sputtered and proved useful while making the target. 


\subsubsection{Results and Discussions}

Sputtering of bismuth telluride and antimony telluride targets was carried out. Table 1 shows the different parameters changed during sputtering of bismuth telluride target.

Table 1: Bismuth telluride sputtering conditions and results

\section{Sputtering conditions and results}

\begin{tabular}{lccccccccc}
\hline $\begin{array}{c}\text { Sampl } \\
\text { e ID }\end{array}$ & $\begin{array}{c}\text { Power } \\
\text { (Watt) }\end{array}$ & $\begin{array}{c}\text { Bias } \\
\text { (Watt) }\end{array}$ & $\begin{array}{c}\text { Thicknes } \\
\text { s (nm) }\end{array}$ & $\begin{array}{c}\text { Time } \\
\text { (min) }\end{array}$ & $\begin{array}{c}\text { Depositio } \\
\text { n Rate }\end{array}$ & $\begin{array}{c}\text { 4 probe } \\
\text { resistance } \\
\text { (ohms) }\end{array}$ & $\begin{array}{c}\text { Sheet } \\
\text { Resistance } \\
\text { (ohms/nm) }\end{array}$ & $\begin{array}{c}\text { EDS } \\
\text { Be }\end{array}$ \\
\hline BiTe-1 & 60 & 0 & 2095 & 20 & 104.75 & 111 & 0.052 & 45.87 & 54.13 \\
BiTe-2 & 50 & 0 & 285 & 20 & 14.25 & 61.32 & 0.215 & 39.58 & 60.42 \\
BiTe-3 & 50 & 4 & 98 & 20 & 4.9 & 9.63 & 0.098 & 38.48 & 61.52 \\
BiTe-4 & 50 & 8 & 260 & 20 & 13 & 57.25 & 0.220 & 43.83 & 56.17 \\
\hline BiTe-5 & 50 & 14 & 200 & 20 & 10 & 50.62 & 0.253 & 44.75 & 55.25 \\
\hline BiTe-6 & 50 & 16 & 158 & 20 & 7.9 & 230 & 1.455 & 46.36 & 53.64 \\
\hline
\end{tabular}

The following parameters were kept constant, pressure to 2 mtorr, temperature to $15^{\circ} \mathrm{C}$, working distance to 40, Argon flow to $10 \mathrm{sccm}$ (Standard Cubic Centimeters per Minute) and deposition time to 20 minutes. To begin with the target power applied was $60 \mathrm{~W}$ (Watt), DC power source and zero bias. The thickness of the film obtained was 2 microns in 20 minutes. The film had small agglomerations of the alloy on the surface, Figure 23 shows SEM image of the agglomerations on the deposited film. These agglomeration formations on the surface are not desired for thin films. These were attributed to the high power applied to the target. Heat produced was too much that there were local melting points on the surface of the target. This explains the high deposition rate. Therefore, the power had to be reduced to $50 \mathrm{~W}$ to gain in deposition rate without localized melting of the target. Deposition rate decreases as the power applied is reduced. 


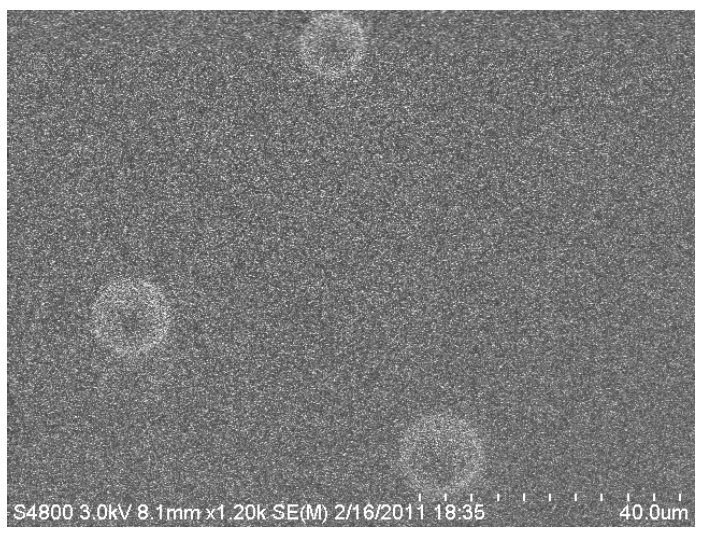

Figure 23: Agglomeration of grains of bismuth telluride sputtered at 60W DC power

The power applied was limited to $50 \mathrm{~W}$, next parameter that could be changed to affect the characteristics of the film was substrate RF bias. Bias was applied for enhanced density of the film, hence better thermoelectric properties and thus contributing to higher mobility of carriers. Figure 24 (a), shows the SEM grain formation at $50 \mathrm{~W}$ without any bias, the film obtained is porous. As the bias power is increased, better density is obtained, but the deposition rate is reduced. To understand the balance between the deposition rate and density of the films, different bias powers were applied. Figure 24 (b), also shows the SEM picture of the film deposited at 50 W DC power with 14W RF bias power. Better density was obtained when substrate bias was applied. Film porosity reduced to almost zero.
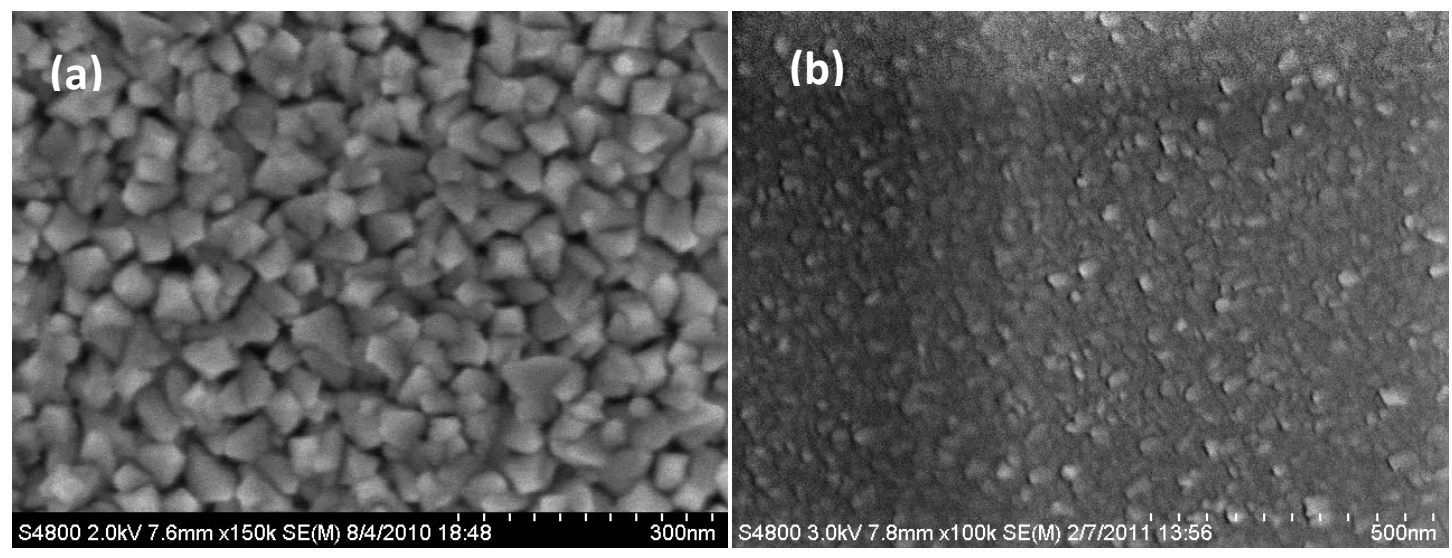

Figure 24: (a) Porous film of bismuth telluride sputtered at 50W DC power with no bias, (b) Denser film of bismuth telluride sputtered at 50W DC power with $14 \mathrm{~W}$ RF bias power 
The EDS data in Table 1, shows the individual atomic concentrations of bismuth and tellurium.

The sputtered sample BiTe-2 shows the stoichiometric closest to the desired stoichiometry. Another trend observed, as bias power is increased tellurium atomic concentration reduces. Thickness of the film over deposition time gives deposition rate.

Table 2 shows the sputtering conditions and results for antimony telluride target. Similar to bismuth telluride target we had agglomeration of the grains, at $60 \mathrm{~W}$ DC power. Localized melt of the target was observed, thus power had to be reduced. At $40 \mathrm{~W}$ sufficient growth rate without any grain agglomeration was obtained. The deposition rate was increased from $30 \mathrm{~W}$ to $40 \mathrm{~W}$ for better deposition rate. The best stoichiometric value obtained was for SbTe-6, sputtered at $40 \mathrm{~W}$ with $8 \mathrm{~W}$ bias power.

Table 2 : Antimony telluride sputtering conditions and results

\section{Sputtering conditions and results}

\begin{tabular}{lllllcccccc}
\hline $\begin{array}{l}\text { Sample } \\
\text { ID }\end{array}$ & $\begin{array}{l}\text { Power } \\
(\mathbf{W})\end{array}$ & $\begin{array}{l}\text { Bias } \\
(\mathbf{W})\end{array}$ & $\begin{array}{l}\text { Thick } \\
(\mathbf{n m})\end{array}$ & $\begin{array}{l}\text { Time } \\
(\mathbf{m i n})\end{array}$ & $\begin{array}{c}\text { Deposition } \\
\text { Rate }\end{array}$ & $\begin{array}{c}\text { 4 Probe } \\
\text { resistance } \\
(\mathbf{o h m s})\end{array}$ & $\begin{array}{l}\text { Sheet } \\
\text { resistance } \\
(\mathbf{o h m s} / \mathbf{n m})\end{array}$ & \multicolumn{2}{c}{ SDS } & Te \\
\hline SbTe-1 & 60 & 0 & 1114 & 10 & 111.4 & 306.75 & 0.275 & 45.29 & 54.71 \\
\hline SbTe-2 & 30 & 0 & 120 & 20 & 6 & 48.76 & 0.406 & 35.41 & 64.59 \\
SbTe-3 & 40 & 0 & 181 & 20 & 9.05 & 58.65 & 0.324 & 37.94 & 62.06 \\
\hline SbTe-4 & 60 & 0 & 2016 & 20 & 100.8 & 353.81 & 0.175 & 43.24 & 56.76 \\
SbTe-5 & 50 & 0 & 1756 & 20 & 87.8 & 260.56 & 0.148 & 42.65 & 57.35 \\
\hline SbTe-6 & 40 & 8 & 170 & 20 & 8.5 & 40.56 & 0.238 & 39.65 & 60.35 \\
\hline
\end{tabular}

Four point probe resistance measurements were done for the binary target thin films sputtered.

The resistance values were directly obtained from four point probe electrical resistance method mentioned in chapter 2. Sheet resistance was calculated from equation 3.1. 


$$
\text { Sheet resistance }=\frac{\text { Resistance }(4 \text { point probe measurement })}{\text { Thickness of thin film }}
$$

Sheet resistance obtained for bismuth telluride target was lowest for BiTe-3, when sputtered at $50 \mathrm{~W}$ and $4 \mathrm{~W}$ bias, the stoichiometry of this film was very close to 40:60 ratio. However, for antimony telluride target, the lowest sheet resistance value was obtained for samples SbTe-4 and SbTe-5, sputtered at high powers of $60 \mathrm{~W}$ and $50 \mathrm{~W}$. These samples were $2 \%$ to $3 \%$ off from the stoichiometric value.

All these experiments were carried out to understand the characteristics of the elements during sputtering. Since, these being preliminary data, further experimentation and analysis should be done to ensure repeatability, optimize for the best conditions.

To better understand the feasibility of this approach, a single composite thin film was sputtered to form the composite thin film of bismuth antimony telluride. Binary target alloy bismuth telluride was sputtered using the parameters of sample BiTe-2 and antimony telluride employing SbTe-6. Sputtering of the two targets was done alternatively. First a layer of bismuth telluride was sputtered and subsequently a layer of antimony telluride was sputtered. The growth rate for the film was low and the sputtering target could be used for sputtering a maximum of 20 hours, after which new targets had to be replaced. With deposition rates of maximum $12 \mathrm{~nm}$ per minute, it was concluded that obtaining films of at least 100 micron would be time consuming. Therefore, it was decided to focus the work towards making thin bulk samples. 


\section{CHAPTER IV}

\section{THERMOELECTRIC MULTILAYER FABRICATION}

\subsection{Introduction}

The best materials to use for power generation and refrigeration near room temperature application are bismuth telluride and alloys [16].

Recent advance in science like nanotechnology can be used to further improve performance of thermoelectric materials. As mentioned in chapter 1, nano structuring of thermoelectric elements enhance the thermoelectric properties. Similar methods of ball milling and hot pressing the thermoelectric materials as followed by Poudel et al and Mehdizadeh et al are followed. Subtle variations are, the process commences from elemental powders than from commercially available ingots (compared to Poudel et. al. [11])and the ternary alloy of bismuth antimony telluride, instead of the composite (compared to Mehdizadeh et. al. [32]) is used for the experiments. Discussed in the following sections are two methods of powder alloy formation from the powders and two methods of consolidation of the powders into bulk samples.

\subsection{Objective}

In order to make the thermoelectric module proposed in chapter 1, individual samples of p-type bismuth antimony telluride and n-type bismuth selenium telluride had to be formed. These were then to be mated to obtain the final thermoelectric module. Figure 25 (a) shows an enlarged image of the individual components of the thermoelectric module, the green color shows the p- 
type thermoelectric leg and (b) pink color shows the n-type thermoelectric leg, copper is represented by orange in both (a) and (b). Figure 25 (c) shows the cross-section image of the thermoelectric module.

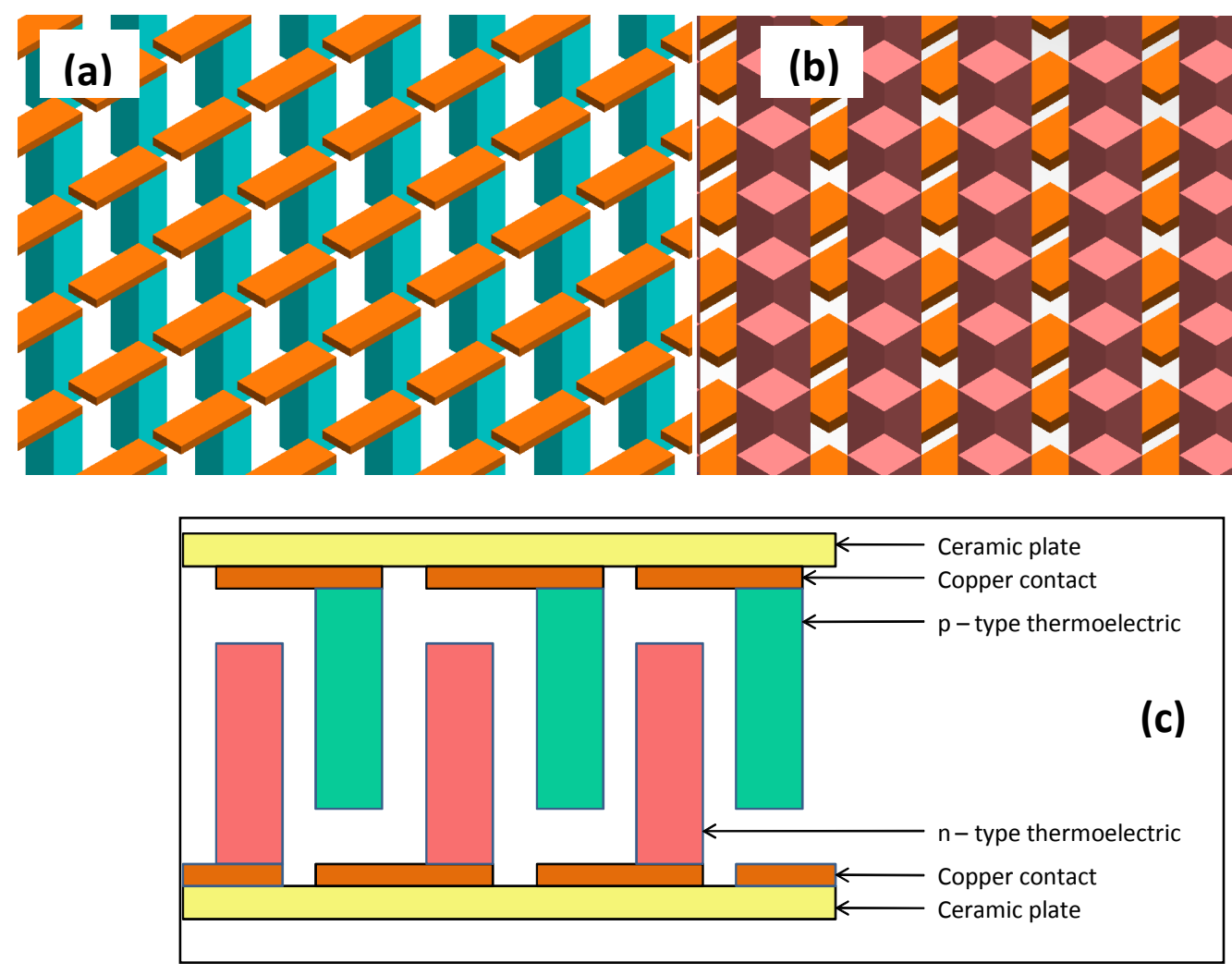

Figure 25 : (a) P-type thermoelectric with copper contact on top, (b) n-type thermoelectric with copper contact at bottom, (c) 2-D image showing the cross-section of module

To obtain such a structure, a sandwich structure of copper, barrier layer and thermoelectric was to be fabricated and processed later with dicing tools. Figure 26 shows the cross-section of the thermoelectric sample to be fabricated. The sample pressed will be 2 inches in diameter. The following sections discuss the processes of fabrication of the 2 inch diameter circular thermoelectric material with diffusion barrier layer and contact metal layer. This was successfully done for the p-type thermoelectric material to some extent. Similar method can also be extended for the fabrication of the n-type bismuth selenium telluride thermoelectric sample. This method of preparation is simple, economically viable and can be extended to other thermoelectric materials, 
if the proper diffusion barrier layer material can be used. In addition to higher ZT values and isotropic properties, the nanostructured bulk materials also possess superior mechanical properties making them more suitable for fabrication and integration [11].

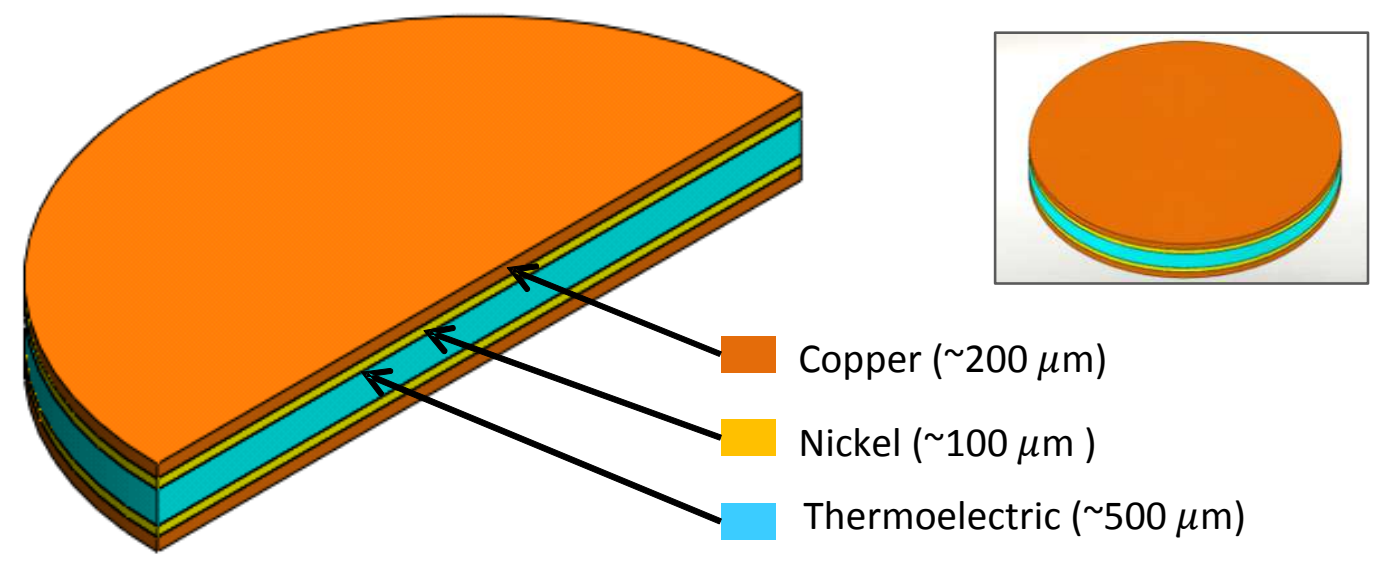

Figure 26 : Cross-section of p-type thermoelectric; Schematic of 2 inch sample (inset)

\subsection{Thermoelectric bulk material}

\subsubsection{Preparation of Alloy powders}

Elemental powders with $99.99 \%$ purity were used for all the experiments. The powders were purchased from Alfa Aesar. Bismuth, Antimony and Tellurium had a mesh size of 200 micron, while Nickel powders of 300 micron mesh spherical balls and copper powder of 625 micon mesh was used for processing.

Primarily two methods of alloy formation were followed. In both the methods of alloy formation mentioned, the binary alloys are formed separately. Bismuth telluride and antimony telluride alloys were individually formed. Under the same milling conditions it would be more time and energy consuming to start from all three powders from the beginning to form the alloy [46]. Moreover, this method allowed the degree of freedom over the stoichiometry of the final ternary alloy. Therefore the alloy powders were produced separately. Binary alloy bismuth telluride, by 
itself is a p-type thermoelectric, however, we add n-type antimony telluride to made bismuth antimony telluride alloy to enhance the thermoelectric properties by alloying [47]. The binary alloys were later milled together in appropriate proportions to form the final ternary alloy based on the chemical formula $\left(\mathrm{Bi}_{2} \mathrm{Te}_{3}\right)_{x}\left(\mathrm{Sb}_{2} \mathrm{Te}_{3}\right)_{1-x}$. The value of $x$ could be varied.

\subsubsection{Mechanical Milling and Annealing}

The elemental powders were weighted according to the atomic stoichiometric ratio, manually mixed and loaded into a stainless steel jar with stainless steel balls. All powder loading operations were carried out in a glove box in argon inert environment. These powders were milled and alloyed using a vibratory mill. Mechanical alloying happens through solid state reaction [20]. The number of balls used for alloy formation and milling had to be appropriate. A trial and error method was followed because, too many balls could lead to sticking of the powders onto the walls of the stainless steel jar. Bismuth, because of it low melting point, has a tendency to stick to the walls and on further ball impact will become hard and cannot be removed from the walls of the stainless steel jar. Fewer numbers of balls could lead to inefficient alloy formation. Mechanical milling achieves nano structuring of the thermoelectric powders. The grain size of the powders was reduced to nano level. Milling gives rise to two effects, the elemental powders are alloyed to form the binary or ternary alloy and also leads to grain size reduction. Alloys are formed because of the impact based contact and the grain size is reduced because of the friction based interaction between the powder and the balls.

Sticking of the powders on the walls of the jar was observed, when elemental powders still remained unalloyed in the mixture. For reduction on time for formation of alloy powders, the powders were annealed at suitable temperatures inside a muffled furnace. Diffusion of elements into each other to happens during the annealing process. Annealed powders are then again milled. This process of annealing and milling can be optimized to make sure, the alloy powders are formed quickly and uniformly. Figure 27, shows the picture of the high energy vibratory mill and 
stainless steel jar used for alloying and nano structuring the elemental powders.
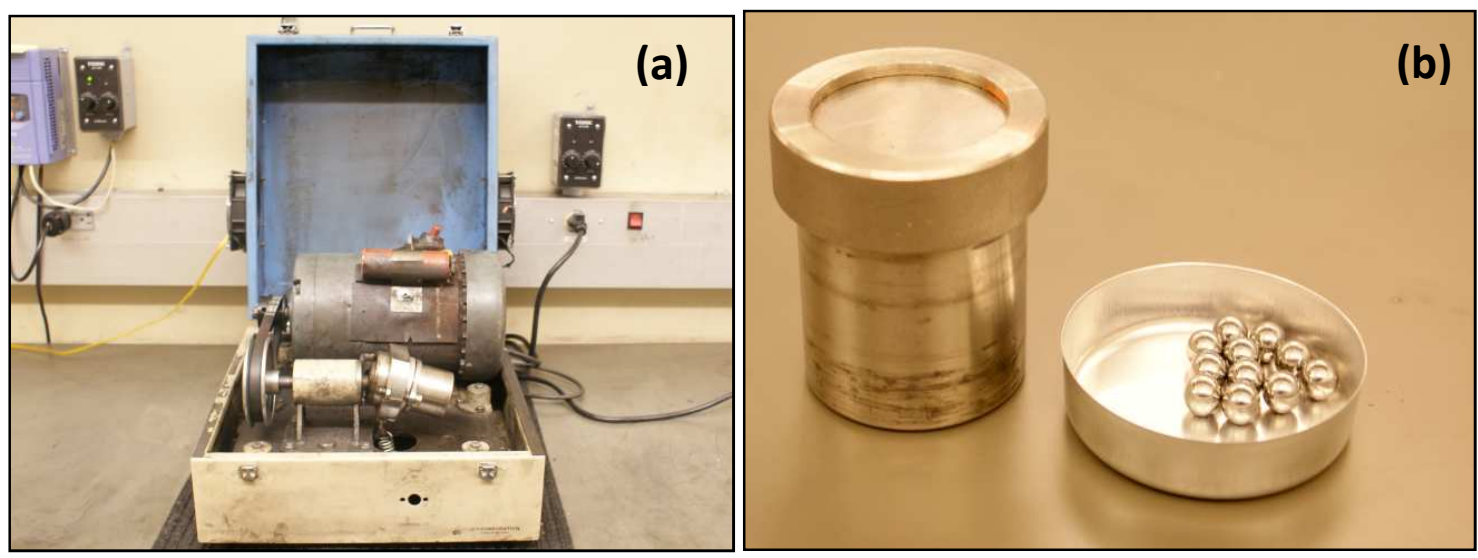

Figure 27 : (a) High energy mill, (b) Stainless steel jar used for alloying the elemental powders

Annealing of the powders has to be done after sufficient micro-mixing has been achieved in the mixture, else it could lead to non homogeneous mixture. The process of annealing must also be carried out at an optimum temperature and the holding/sintering time (annealing) of the powders at different temperatures plays a vital role in the formation of the alloy faster. The temperature cycle was decided by annealing the powders and analyzing the powders in XRD. Therefore, it is a very integral that the XRD of the powders were to be obtained after very process of milling and annealing. A rapid annealing will lead to melting of the elemental powder into one chunk, which will have to be broken and the stoichiometric of the powder could be inconsistent. The crystal size of the alloy powders was determined from powder XRD and the grain size SEM images.

Typical case of alloy formation of bismuth telluride involved the following steps, 50gms of bismuth and tellurium powders suitably mixed according to the stoichiometric ratio of 40:60 were weighted out, manually mixed, milled and alloyed using 5 steel balls of $10 \mathrm{~mm}$ diameter. Milling for about $24 \mathrm{hrs}$ ensured proper alloying and nano-structuring of the powder. Powders were annealed after 12 hours of milling for 3 hours before continuing further milling. The annealing steps are as follows, RT (Room Temperature) to $250^{\circ} \mathrm{C}$ in 30 minutes, holding at $250^{\circ} \mathrm{C}$ for 1 
hour, ramping up to $350^{\circ} \mathrm{C}$ in $1 \mathrm{hr}$ and holding at $350^{\circ} \mathrm{C}$ for $1 \mathrm{hr}$, cooled to RT. After suitable binary alloy formation was ensured, they were mixed in the ratio of $1: 4$ to form $\left(\mathrm{Bi}_{2} \mathrm{Te}_{3}\right)_{0.2}\left(\mathrm{Sb}_{2} \mathrm{Te}_{3}\right)_{0.8}$. The ratio of 1:4 showed the best thermoelectric properties.

Similar process was followed for the alloy formation of antimony telluride, in a new stainless steel jar. However, the annealing steps followed were as follows, RT to $370^{\circ} \mathrm{C}$ in 1 hour, holding at $370^{\circ} \mathrm{C}$ for $1 \mathrm{hr}$ and cooling back to RT.

\subsubsection{Induction furnace ingot milling}

Another efficient method of alloy formation reducing the time taken for alloy formation was achieved by fabrication of the alloy ingot using an induction furnace setup as previously mentioned in chapter 3 . The powders milled by this method showed better electrical properties as compared to the previous mechanical milling and alloying method.

Electromagnetic induction in an induction furnace gives rise to eddy currents. Joule heat is created inside the material due to resistance to eddy currents. A high frequency AC is passed through the electromagnet for heat generation in the induction furnace. Temperature is controlled by the amount of current passed. The melting points of bismuth, antimony, tellurium are 271.5 ${ }^{\circ} \mathrm{C}, 630.63{ }^{\circ} \mathrm{C}, 449.51{ }^{\circ} \mathrm{C}$ respectively [48].

During induction furnace operations, extra tellurium was added into the powder mixture, due to the loss of Tellurium. In a typical case of alloy formation of bismuth telluride alloy, the powders were mixed in the atomic ratio of $36: 64$, increasing the tellurium ratio by $4 \%$. The induction furnace melting was done for 30 minutes, by varying the current. A temperature of about $700 \mathrm{C}$ was maintained. Figure 21, shows a schematic and picture of the induction furnace setup used. Error! Reference source not found. shows the picture of an as received ingot from the induction furnace. 


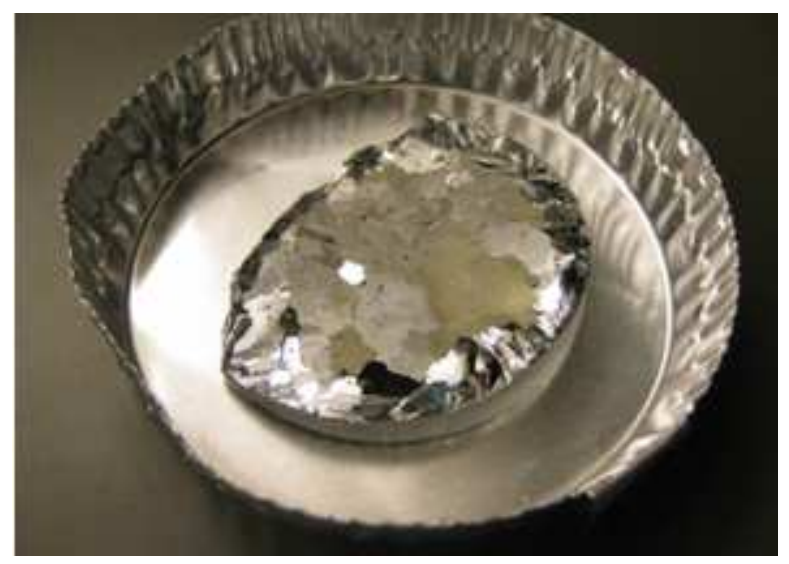

Figure 28: Picture of as obtained induction furnace ingot of $\mathrm{Sb} 2 \mathrm{Te} 3$

The polycrystalline bulk alloy obtained through this method has to be milled into small grains to obtain nano structuring. Ingot was broken manually into small chunks and then loaded into the stainless steel jar for further grain size reduction. This process was done both for bismuth telluride and antimony telluride alloy.

Individual powders of bismuth telluride and antimony telluride were obtained and mixed in the required alloy ratio. They were milled together to form the bismuth antimony telluride (p-type) ternary alloy. The powders where mixed to obtain $\left(\mathrm{Bi}_{2} \mathrm{Te}_{3}\right)_{0.2}\left(\mathrm{Sb}_{2} \mathrm{Te}_{3}\right)_{0.8}$.

SEM images, EDS data and XRD analysis were done, after alloy formation and milling to confirm homogeneity and alloy formation. Important information that the XRD revealed was the presence of the oxides and the crystal size. Figure 29 shows the SEM image of the final alloy powder formed. A grain with the size of $35 \mathrm{~nm}$ is shown. The stoichiometric ratio of 40:60 was confirmed by carrying out EDS analysis on a sample of the alloy powders. 


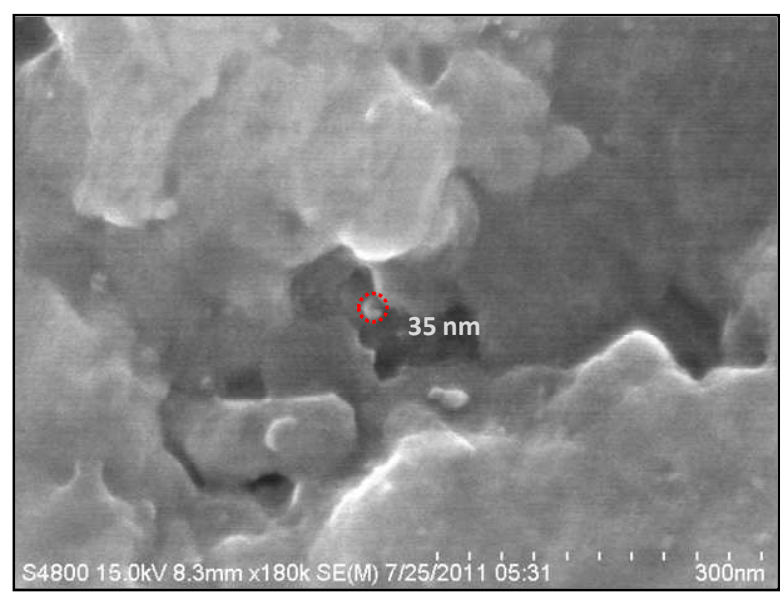

Figure 29 : SEM image of final alloy powder used for experiments

XRD analysis of the binary bismuth telluride, antimony telluride and the final bismuth antimony telluride powders were obtained. The XRD plot of intensity vs. 2-theta is shown in Figure 30. Plot "a" shows the peaks of binary bismuth telluride alloy powder, "b" binary antimony telluride powder and "c" the ternary bismuth antimony telluride alloy powder.

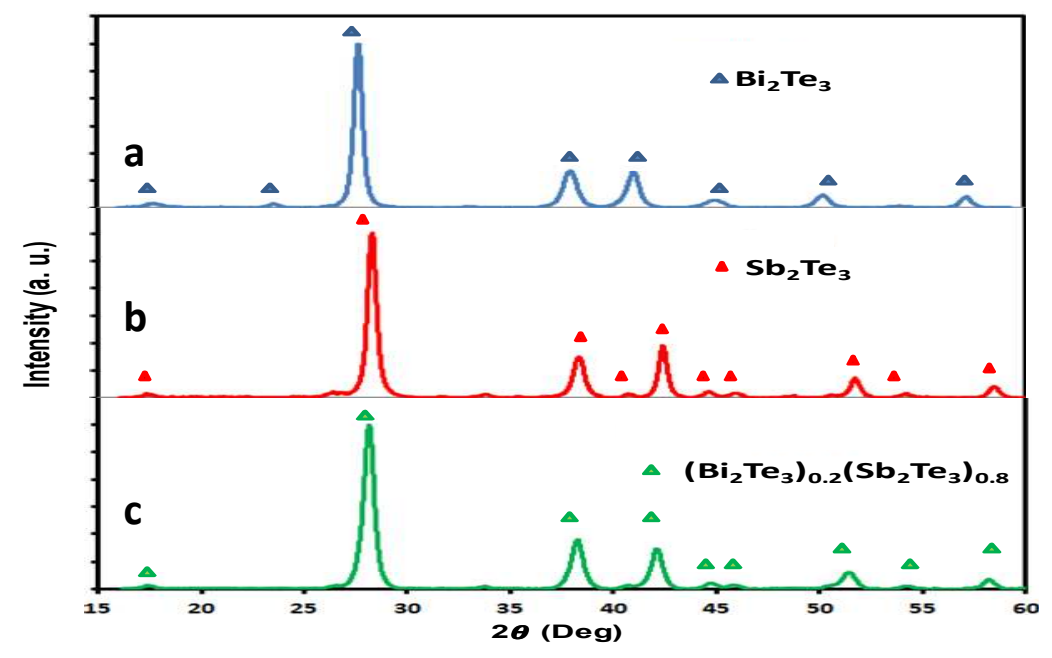

Figure 30: XRD plot of the alloyed powders used for experiments

Care must be taken to carry out all powder mixing and ingot handling are done at controlled environments. Contamination of powders due to foreign materials must be avoided. Another 
important factor that affects thermoelectric properties of the these alloys is the presence of oxygen [30]. Oxidized powders showed decrease in thermoelectric characteristics.

\subsubsection{Consolidation of nanopowders}

The thermoelectric powders obtained after alloying, were to be pressed and sintered at suitable high temperatures to obtain the bulk material. Figure 31, shows pictures of the initial state of the powder and final shapes after they are processed. Figure 31(b), shows picture of the sample diced and polished for ZEM and Laser flash measurements. Figure 31 (C) shows a picture of multilayered sandwich thermoelectric sample pressed.
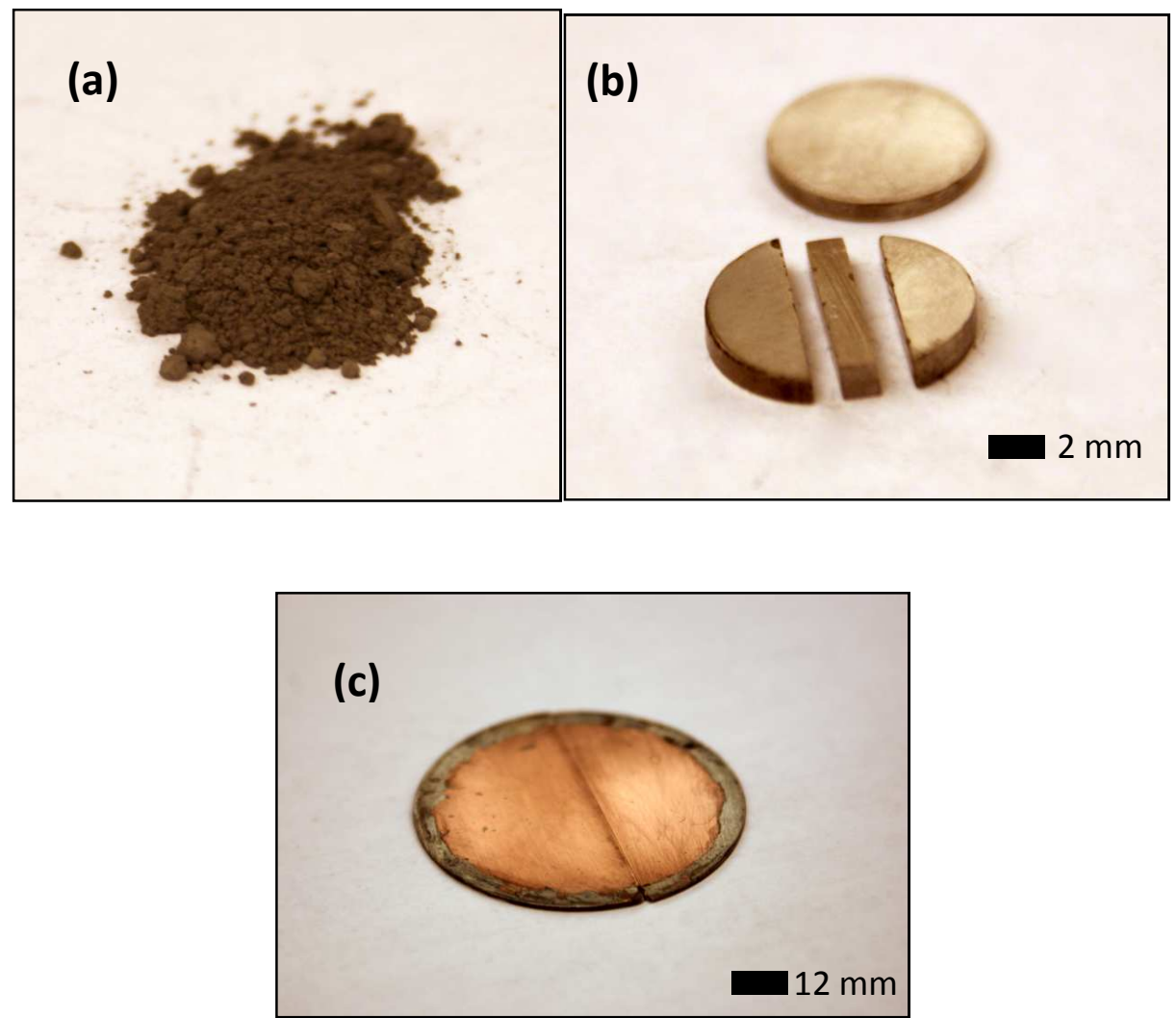

Figure 31 : (a) Alloyed powders ready to be pressed and sintered, (b and c) Sample obtained after consolidation process 
To fabricate thermoelectric modules, we had to compact the nano-powders into bulk shape. Two different methods of powder compaction were employed for compaction of the powders, Spark Plasma Method (P2C) and Induction furnace hot pressing.

\subsubsection{Spark Plasma Sintering}

The nano powders to be pressed and sintered are loaded into a graphite die and DC current is passed through. Graphite being highly electrically conductive provides least resistance. Whereas the powders heat up owing to greater resistance provided to the flow of the current. Figure 32 shows a schematic representation of the spark plasma sintering process. Current was passed through the copper plates. This method was used for sintering the alloy powders for fabricating the target for thin film applications. A Carver, hydraulic hot press was used for applying load for compaction.

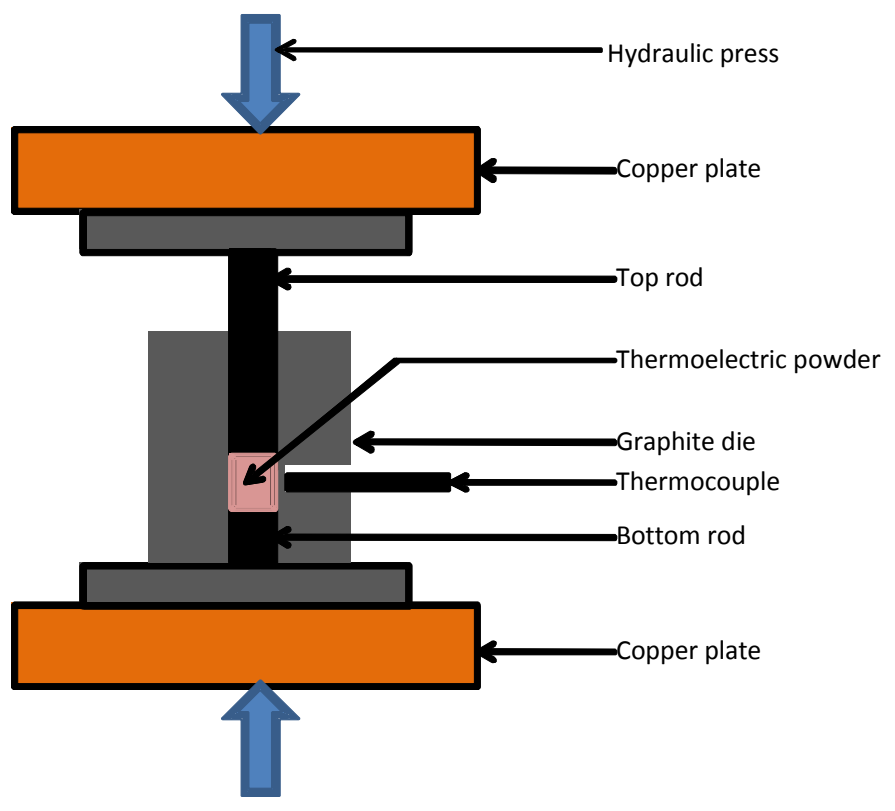

Figure 32: Schematic Spark plasma sintering setup used for alloy powder compaction 
The powders to be sintered at preloaded into the die, in a glove box. A hole is drilled into the die, for inserting the thermocouple to estimate the approximate the temperature of the sample. There is a temperature difference between the powders in the graphite die thermocouple temperature, which here is neglected.

\subsubsection{Induction Furnace hot pressing}

As mentioned previously, employing the induction furnace is a quick and efficient process both in the matter of alloy ingot formation and sample sintering.

The alloy powders are vulnerable to oxidation, the moment they are removed from the glove box. Hot pressing using the spark plasma sintering method (P2C) is relatively a slower process compared to induction furnace. The current cannot be ramped up rapidly as this will lead to localized heating up and melting of the powder sample. These issues are overcome using the induction furnace. Figure 33 gives a schematic of the induction furnace hot pressing setup used. A homemade hydraulic press setup was used for compacting the powders, in this case.

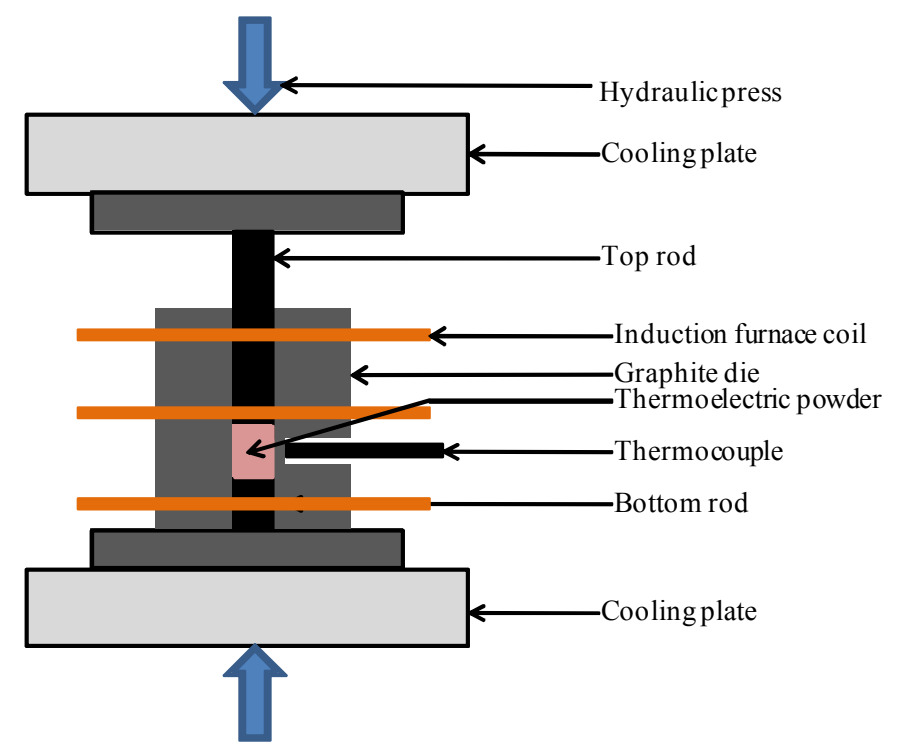

Figure 33 : Schematic of induction furnace hot pressing 
The basic setup remains the same as in $\mathrm{P} 2 \mathrm{C}$ process. However, here the powder loaded graphite die was placed inside induction furnace copper coils. On application of current, electromagnetic flux was generated around the whole graphite die, therefore the powders get uniformly heated. Ramp rate for heating is not an issue since, the entire sample was heated at the same rate. Cooling plates at the top and bottom are provided, so that the hydraulic press stage does not heat up and temperature can be controlled. Cold pressing of all sample powders are done, before hot pressing to obtain more densification. Greater density enhances the electrical conductivity.

\subsubsection{Fabrication of P-type multilayered thermoelectric sample}

The samples were all pressed in a 2 inch diameter hole graphite die. As a preliminary test, a sample of thermoelectric powders alone was pressed, using the induction furnace in the 2 inch diameter graphite die. Problem encountered was, the thermoelectric sintered sample stuck to the surface of the top graphite rod and had to be cut and polished. However, this problem was overcome with the introduction of the metal and diffusion layers on the top and bottom.

To fabricate the thermoelectric elements with contact metals, unlike the conventional method of depositing or growing layers of diffusion layer or metallic contacts, we have compacted it on the top and bottom of the thermoelectric powder layers. This is a novel method tired for making multi-layers of thermoelectric, barrier layer and metal layer at once.

The diffusion and metallic contact layer powders were loaded into the graphite die and hot pressed. This method completely eliminates the post processing step of coating diffusion layers and also ensures a larger and better contact area between each layer. Contact resistance, if not addressed properly, will lead to loss of energy due to voltage drop across the metal layers.

To fabricate a thermoelectric sample, first a layer of copper powder was loaded and flattened out on the bottom rod of the graphite die. These process were carried out in an argon filled glove box to prevent oxidation of the powders. A hardened stainless steel 2 inch diameter rod was used to 
flatten the copper layer. The hardened stainless steel rod was inserted very slowly, to prevent the copper powder from exiting out of the die, owing to the fineness of the copper powder and vacuuming effect created. Stainless steel rod was snug fit in the hole, for smooth traverse in and out of the hole. Once inserted the stainless steel was rotated very slowly in a random manner both clockwise and anticlockwise. Figure 33 shows the stainless steel loaded graphite die.

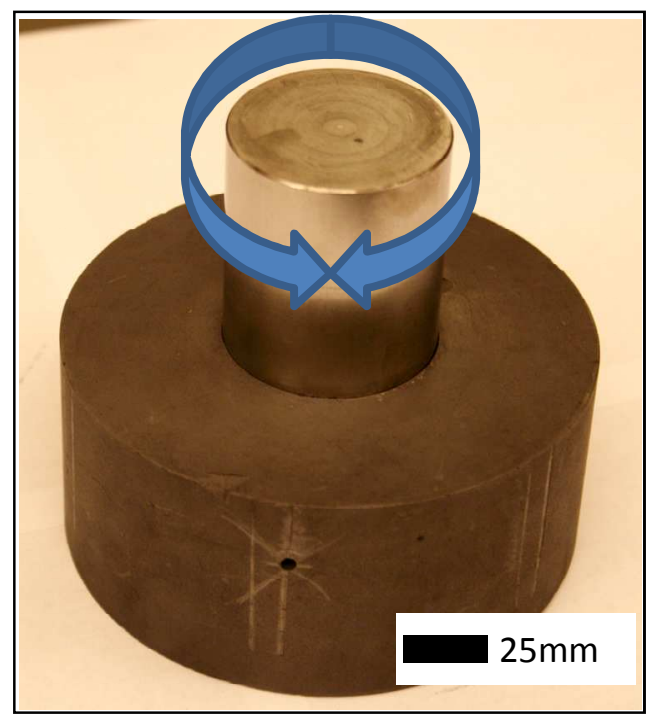

Figure 34 : Picture of powder loaded graphite die loaded with stainless steel rod, the arrows represent the shearing motion needed for flattening the powders

The copper powder flats out perfectly on a micro scale, because of the rotational shearing force and weight of the stainless steel rod. The degree of flatness of each layer depends upon the smoothness of the stainless steel rod. To ensure the maximum smoothness, the rod was polished to a mirror finish. The die along with the steel rod was vacuum packed and taken out of the glove box to be cold pressed. The cold press was done at 6 tons, with a hold time of 5 minutes. After, the cold press, the die was inserted into the glove box again and the steel rod was removed slowly after cold press. Before removing the steel rod, a slight shearing motion is given again, to ensure that there is no copper sticking on the steel rod. The removed steel rod is polished again to mirror finish and ultra-sonicated cleaned for 15 minutes to remove any copper powder residue left 
behind. A smooth and flat finish is ensured so that cross-section of the sample remains the same and contact resistance is decreased.

Following the metal contact layer of copper, is the diffusion layer of nickel powder was loaded into the die. The same steps followed for the initial layer of copper was again repeated for nickel and thermoelectric powder layers. Figure 35 shows the picture of the smooth layers after flattening, compaction and cold pressing.

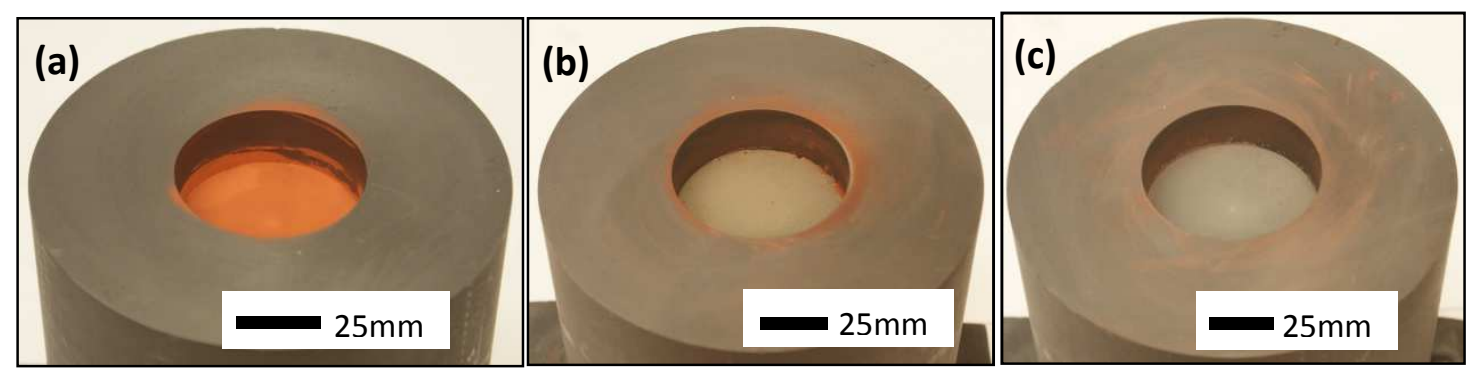

Figure 35: Picture of different powder layers after flattening, compaction and cold pressing,

( a) Copper, (b) Nickel, (c) Thermoelectric powders

Upon completion of loading all the 5 layers of the sandwich structure and cold pressing, steel rod was removed and replaced with a 2 inch diameter graphite rod. Cold pressing is again carried out. The graphite die was ready to be sintered. Sintering is done using the house made induction furnace hydraulic press.

In a typical case, powders of the 5 layered sandwich structure after loading into the graphite die was placed in the induction furnace hot press. The sample was pressed a temperature of $600^{\circ} \mathrm{C}$ for 1 minute (holding time/sintering) at 800 psi pressure. Figure 36 shows picture of a sectioned view of a multilayered thermoelectric sample. The thickness of the thermoelectric layers was $1 \mathrm{~mm}$. 


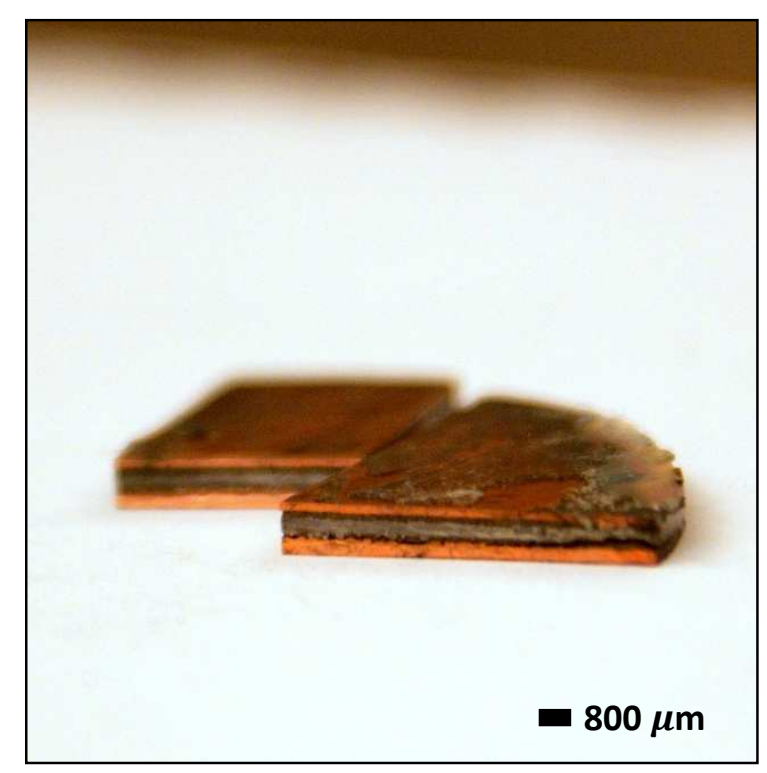

Figure 36: Cross-section of a sandwich structure pressed

The electrical conductivity and Seebeck co-efficient of such a multilayered sample pressed was evaluated using ZEM. The layers of copper and nickel were removed by polishing and samples were obtained for ZEM and laser flash measurements.

\subsubsection{Results and Discussion}

Multi-layered thermoelectric structure with straight layers was obtained. The preliminary test of pressing the thermoelectric powders at $550^{\circ} \mathrm{C}$, yielded in an un-sintered sample. Therefore the temperature was increased to $600^{\circ} \mathrm{C}$. Figure 37 shows the plot of electrical conductivity, Seebeck co-efficient, power factor, thermal conductivity and figure of merit versus temperature of the sample obtained after removal of the copper and nickel layers. Figure 37 shows the plots of the sample compared with the State-of-the-art ingot labeled crystalline. The electrical conductivity of the sample is low when compared and accounts for the lower power factor. However, the Seebeck co-efficient remain almost same. This is a promising start and properties can be further improved by varying the press conditions. 

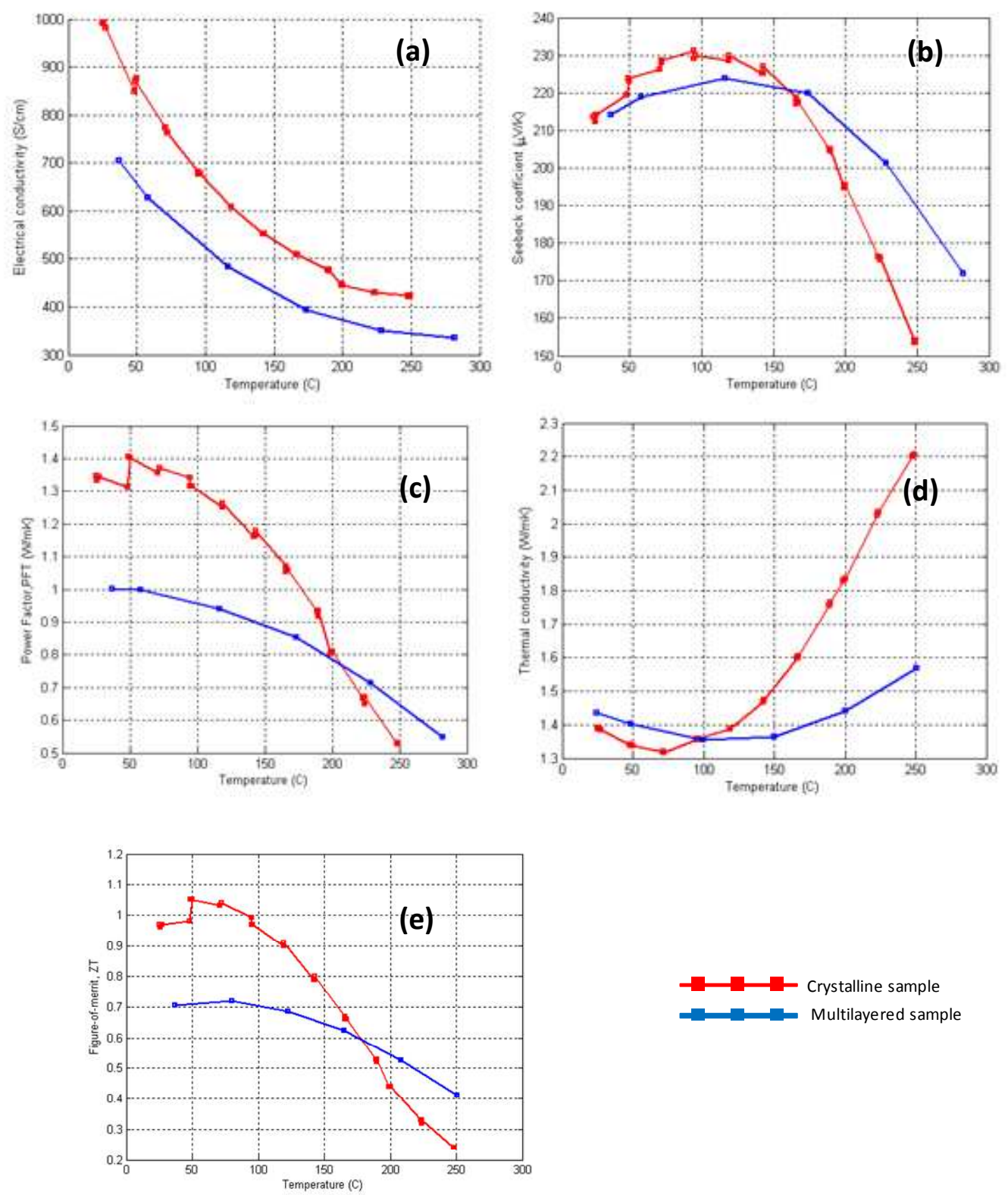

Crystalline sample

Multilayered sample

Figure 37 : Temperature dependence of (a) electrical conductivity, (b) Seebeck coefficient, (c) power factor, (d) thermal conductivity and (e) figure of merit of crystalline industrial sample and multilayered thermoelectric sample

All the samples were pressed at $600^{\circ} \mathrm{C}$. Copper and nickel, though having a high melting point of $1080^{\circ} \mathrm{C}$ and $1480^{\circ} \mathrm{C}$ are well sintered and formed a hard layer which was very desirable for the fabrication of the thermoelectric module. By trial and error method, varying thickness of different 
layers were sintered. Figure 36 shows the picture of a $1 \mathrm{~mm}$ thick thermoelectric multilayer pressed.

A major setback, observed was the poor adhesion of copper to the thermoelectric or nickel layers. A reason that could be attributed to poor adhesion was, copper getting oxidized and formed a greenish oxide layer between nickel and copper at interface. The copper plate could be sheared -off easily from the nickel layers. However, the adhesion between the nickel diffusion layer and thermoelectric was intact. Figure 38 shows a stereoscopic image of the thermoelectric, nickel interface. Nickel powders, because of its, spherical shape tend to produce, more contact surface, between the thermoelectric and barrier layer which can be observed in the SEM image in Figure 39. The absence of the barrier layer gave rise to diffusion of copper into thermoelectric and formation of the copper telluride phase, this can be clearly observed in Figure 38(a).
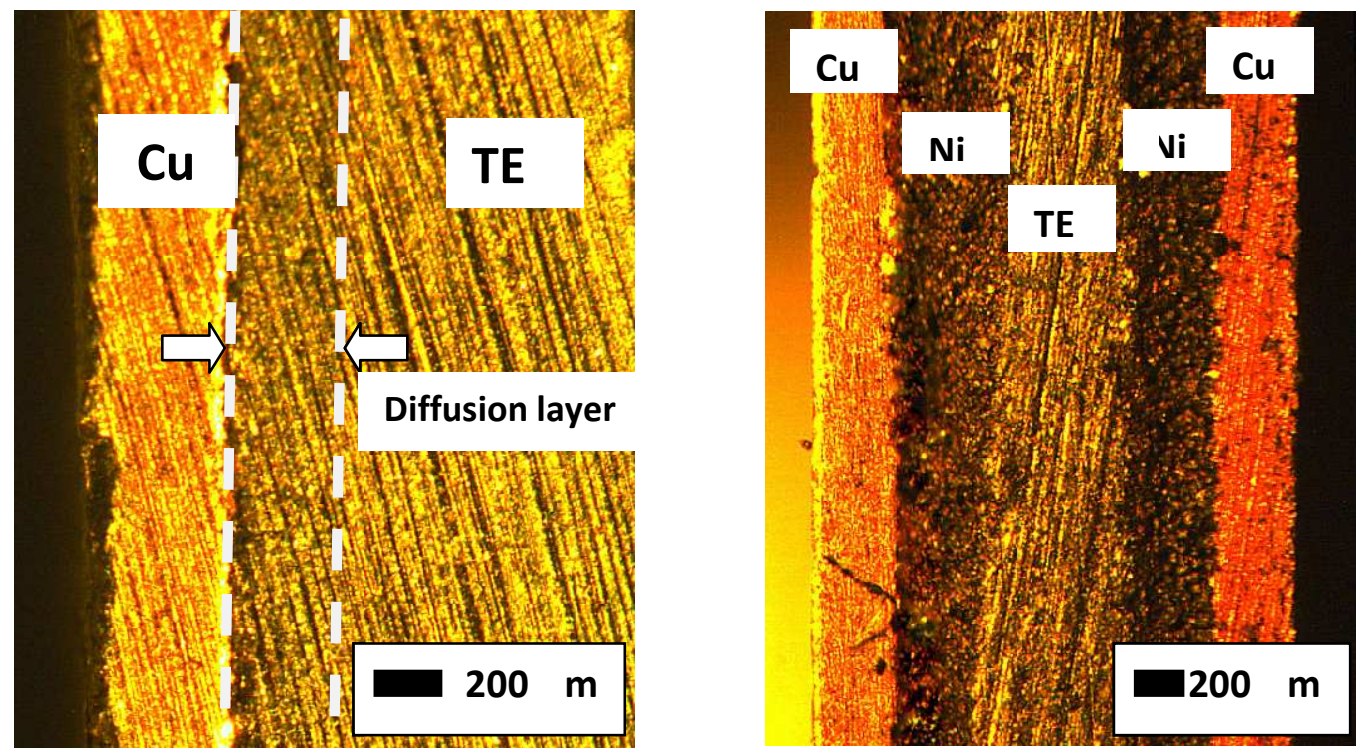

Figure 38: Microscopic image of interface; (a) Discoloration due to Copper diffusion layer into thermoelectric (b) Multilayered structure composite with Nickel diffusion barrier included $(\mathrm{TE}=$ Thermoelectric $)$ 
The EDS line scan was done over the interface and a graph was plotted showing the concentrations of each material into other layers. Three layers are shown in the SEM image in Figure 39(b) the order of the layers from left to right is thermoelectric, nickel and copper.

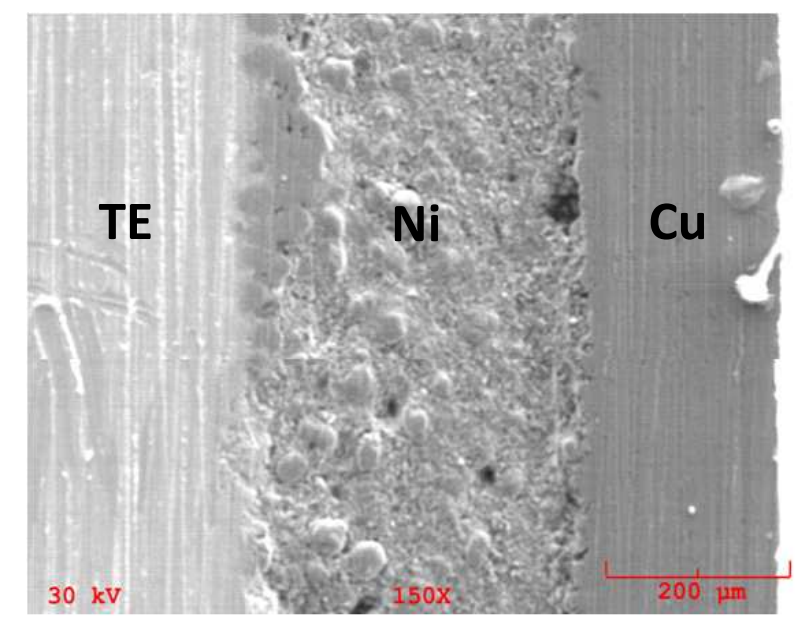

Figure 39 : SEM image of the interface regions, spherical shape Ni can be observed

The EDS line scan was carried out on the thermoelectric, nickel interface. The line scan data was plotted in a graph in Figure 40. The plot reveals some copper diffusion into the thermoelectric, but these can be dismissed as noise. As known before, the nickel diffused into the thermoelectric powders by about $40 \mathrm{~nm}$, which is negligible when dealing with bulk materials. EDS is not a reliable evaluation for determining diffusion. Diffusion can be best studied by using Rutherford backscattering spectrometer and XRD analysis. 


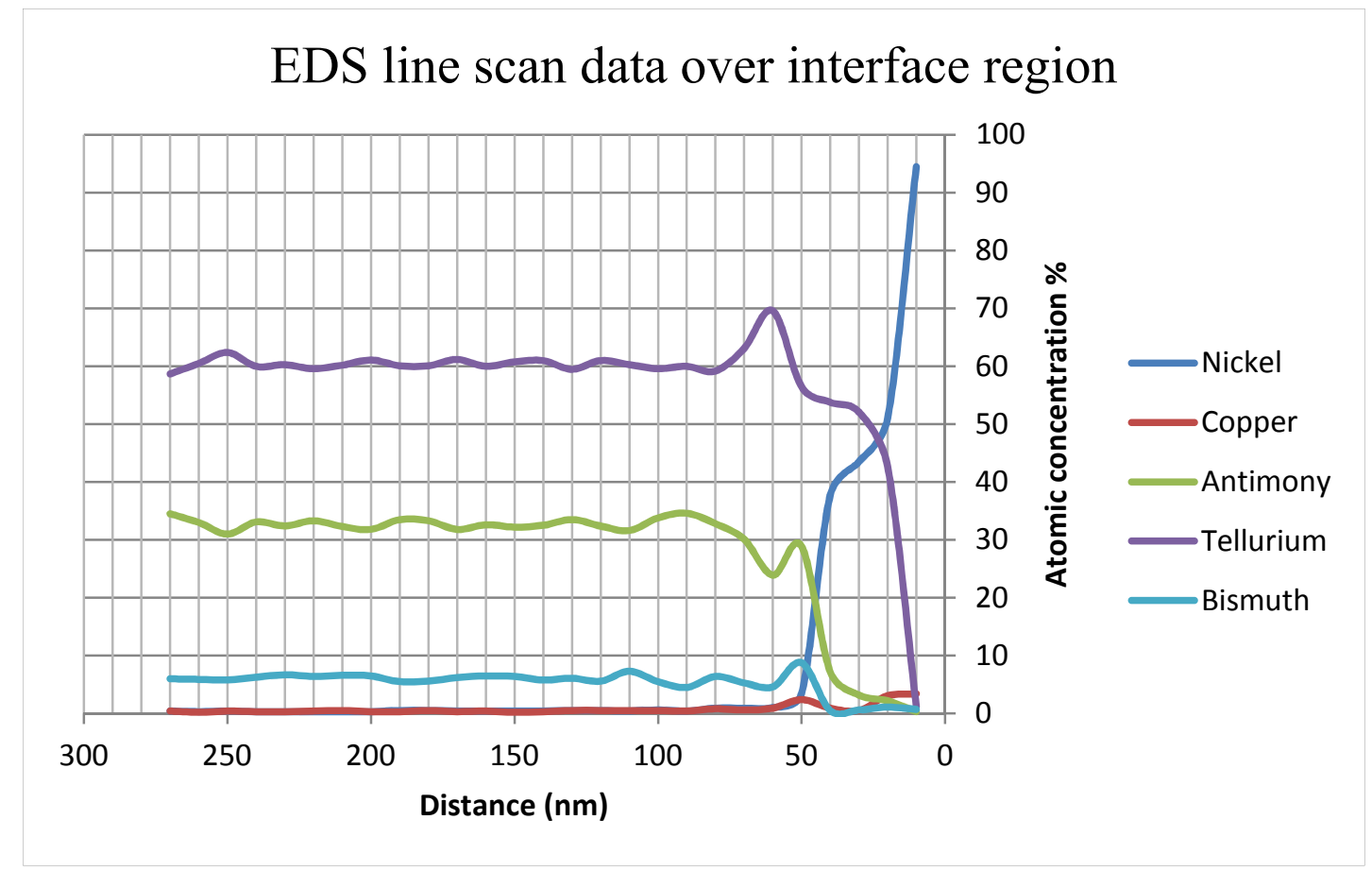

Figure 40 : Graphic representation of the EDS data showing diffusion of the layers 


\section{CHAPTER V}

\section{CHARACTERISATION OF N-TYPE THERMOELECTRIC}

\subsection{Introduction}

The n-type thermoelectric material having great potential for application at room temperature range is the ternary alloy of bismuth, selenium and tellurium [16]. For the thermoelectric module to function efficiently, a combination of both p-type and n-type thermoelectric materials are required. The highest $\mathrm{ZT}$ reported for n-type bismuth selenium telluride single crystals was 0.85 at room temperature. With further the advances in the field of thermoelectrics, the ZT for bismuth selenium telluride improved to 1.04 at $125^{\circ} \mathrm{C}$ for the ternary alloy $\mathrm{Bi}_{2} \mathrm{Se}_{0.3} \mathrm{Te}_{2.7}$ [31]. This improvement in ZT was attained through the increase in the electrical conductivity with a very small increase in thermal conductivity.

\subsection{Objective}

The aim of the experiments carried out with bismuth selenium telluride was to configure the optimum conditions for obtaining enhanced thermoelectric properties. For fabrication of the ntype thermoelectric samples, optimization of the powder alloy and process methods had to be carried out. As a starting point to obtain a basic n-type device the recently reported alloy configuration of $\mathrm{Bi}_{2} \mathrm{Se}_{0.3} \mathrm{Te}_{2.7}$ was investigated. On successful completion of optimization of the powder and process conditions, multilayered thermoelectric samples with a diameter of 2 inches were processed. The samples were to be fabricated with the diffusion barrier layer (Ni) and metal contact layer $(\mathrm{Cu})$ similar to the p-type device as discussed in the previous chapter. 


\subsection{Experimental Procedures}

Experiments were carried out on the alloy powders with the composition $\mathrm{Bi}_{2} \mathrm{Se}_{0.3} \mathrm{Te}_{2.7}$. To further enhance the thermoelectric properties, silicon germanium arsenide ( $\mathrm{SiGeAs}$ ) was added to bismuth selenium telluride. The thermoelectric properties were measured using ULVAC ZEM and Netzsch laser flash and the measurement results are discussed in further sections.

\subsubsection{Preparation of Alloy powders}

Elemental powders of bismuth, selenium and tellurium with $99.99 \%$ purity were used for all the experiments. All the powders were purchased from Alfa Aesar. Bismuth, Selenium and Tellurium of mesh size of 200microns were used for the experimentations.

As previously mentioned in chapter IV, the binary alloys of bismuth selenium and bismuth telluride was prepared separately and mixed in the appropriate alloy proportion. Binary alloys were mixed and mechanically milled together to obtain the final ternary alloy.

Bismuth sellenide alloy was prepared using mechanical milling and annealing. Processing steps of preparation of the binary alloy are as follows. Bismuth and selenium were mixed in atomic stoichiometric ratio 40:60 and the mixture was milled in a vibratory mill for 5 hours with 4 hardened stainless steel balls of $15 \mathrm{~mm}$ diameter. The powders were then annealed at 270 for 3 hours. And again milled for 24 hours. Second cycle of annealing was carried out at $350^{\circ} \mathrm{C}$ for 3 hours. Post second annealing, powders were milled for 24 hours.

Binary alloy of bismuth telluride was prepared using the induction furnace ingot milling as mentioned in chapter IV.

Two batches of 25 grams each ternary of the alloy was milled for the experiments. First batch of powders were labeled "P1" and the second batch as"P2". 
P1 batch was formed by milling the binary alloys for about 24 hours with 3 stainless steel balls of 10mm diameter. Batch P2 was milled for 12 hours and were annealed. First annealing cycle being was carried out at $275^{\circ} \mathrm{C}$ for 15 hours. These were milled for 5 hours and annealed again. Second annealing was $350^{\circ} \mathrm{C}$ for 12 hours. Post second annealing, the alloy powders were milled for 5 hours to obtain the final alloy powders.

Powder P3 was formed by annealing small amount of powder P1 at $350^{\circ} \mathrm{C}$ for 4 hours and continued for milling for 3 hours. SiGeAs was added by $5 \%$ volume of BiSeTe, to powder P2. These were then milled for 10 hours.

\subsubsection{Consolidation of the powders}

The powders were compacted in a similar manner as followed for the p-type material consolidation by hot pressing in induction furnace. Powders were loaded into a graphite die of $12.7 \mathrm{~mm}$ diameter. The convention followed for labeling of the sample is as follows:

"Sample number - Material composition- Powder batch - Annealing time"

The first sample $01-\mathrm{BiSeTe}-\mathrm{P} 1$ was pressed at $490^{\circ} \mathrm{C}$ at a pressure of $400 \mathrm{psi}$. The sample temperature was increased to $490^{\circ} \mathrm{C}$ and immediately cooled to RT. Temperature of $490^{\circ} \mathrm{C}$ was selected based on the previous study carried out on these alloys[11]. The applied pressure was restricted to $400 \mathrm{psi}$ to avoid the cracking of the die. The sample was ejected at RT. A shiny sample with no cracks was obtained. The density measured was $90 \%$ of the theoretical value using Archimedes principle. Traces of melt were observed around the sample 01-BiSeTe which was sintered at $490^{\circ} \mathrm{C}$, probably due to improper alloying. In order to obtain a more alloying of the elements, the powders were milled for extra 24 hours.

The processing steps for all the samples are schematically shown in Figure 41. The pressure was varied for sample 03-BiSeTe-P1 and hot pressed, because of cracking in the die observed at 400psi. Holding/sintering time for this sample was 90 seconds, traces of melt were again 
observed in 03-BiSeTe-P1 due to which the holding temperature in sample 04-BiSeTe-P1 was reduced to $450^{\circ} \mathrm{C}$. Sample $04-\mathrm{BiSeTe}-\mathrm{P} 1$ was pressed at $450^{\circ} \mathrm{C}, 350 \mathrm{psi}$ with holding/sintering time of 90seconds. Samples 03-BiSeTe-P1 and 04-BiSeTe-P1, both yielded comparatively poor properties as discussed later. Therefore, it was decided to follow the course of first sample 01BiSeTe-P1 as it yielded better characteristics. Sample 07-BiSeTe-P2 was pressed at the same conditions of 01-BiSeTe-P1, keeping both the pressure and temperature constant at 400psi and $490^{\circ} \mathrm{C}$. However, the ramp time was delayed by 2 minutes 34 compared to $01-\mathrm{BiSeTe}-\mathrm{P} 1$.

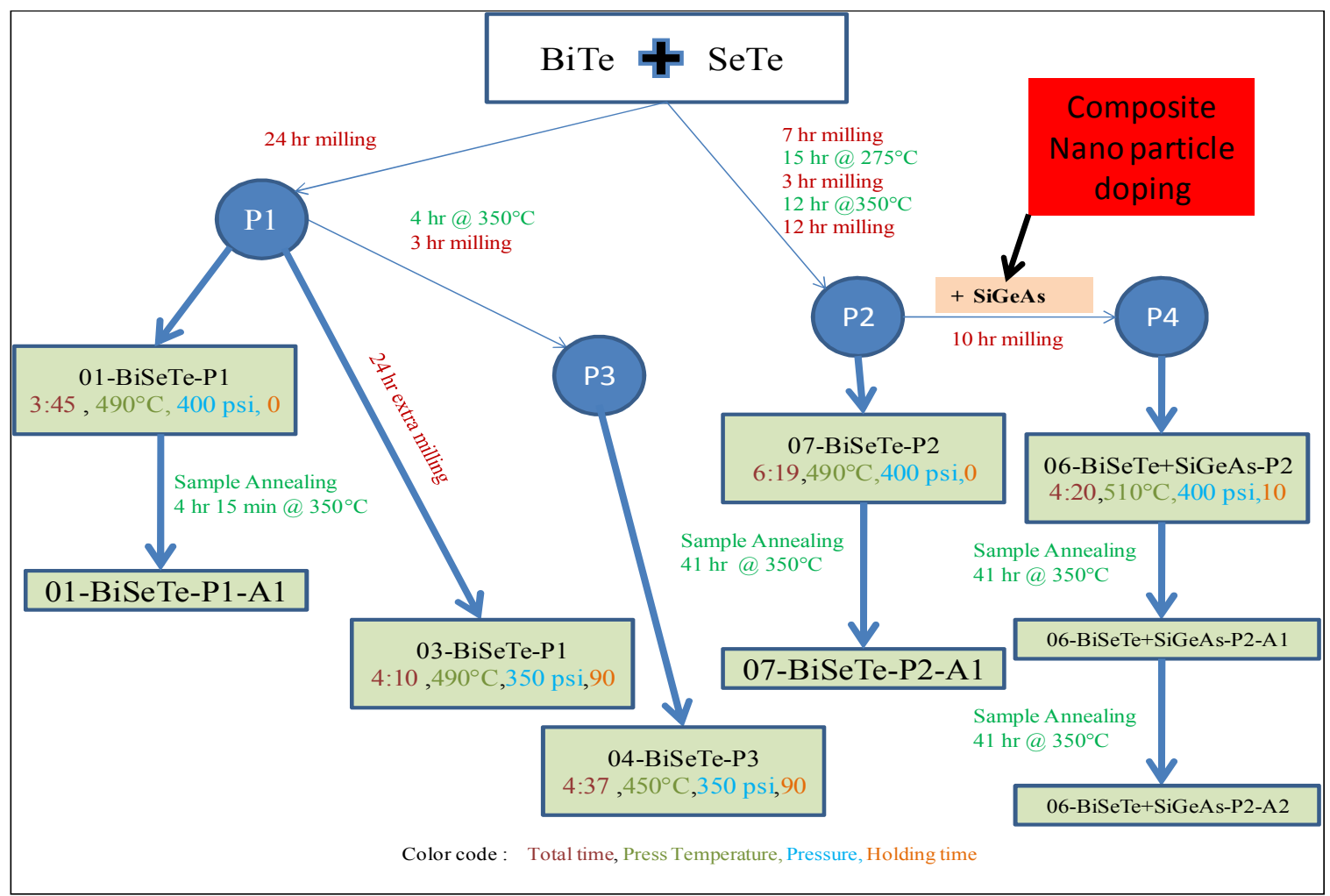

Figure 41: Flowchart showing powder alloying and sample pressing conditions

All the hot pressed samples were cut into rectangular bars for measurement of electrical conductivity and Seebeck coefficient and discs for thermal conductivity measurements. Annealing of samples was performed for various holding/sintering times at $350^{\circ} \mathrm{C}$ in a muffled furnace. 


\subsubsection{Results and discussions}

Doping mechanism in bismuth selenium telluride structures are through structural defects (or deviation from stoichiometry) namely antisites and vacancies. Electrical conductivity, Seebeck co-efficient and thermal conductivity measurements were conducted on the as pressed samples and annealed samples. Power factor and Seebeck coefficients were obtained.

Thermal conductivity $(\mathrm{k})$ is the sum of thermal conductivity contribution from lattice thermal conductivity or phonons $\left(\mathrm{k}_{\mathrm{L}}\right)$ and electronic carriers $\left(\mathrm{k}_{\mathrm{e}}\right)$. Lattices of atoms vibrate and transfer heat, these quantized lattice vibrations are called phonons. The charge carriers can also carry and transfer heat, heat contribution by the carriers is the electronic part of thermal conductivity.

\subsubsection{Effect of annealing}

Figure 42 shows the thermoelectric transport properties, power factor and figure of merit for samples pressed at the same conditions, with different powders and samples annealed for different durations. These samples also showed the highest figure of merit among all the samples in this study.

The electrical conductivity plots of the sample shows the characteristics of a highly doped semiconductor material, which is very desirable for thermoelectric materials. This behavior corresponds to a metal, loss in electrical conductivity with increase in temperature is observed, because of carrier-carrier and carrier-phonon scattering. Seebeck coefficient for highly doped semiconductor can be considered to be directly proportional to the temperature, therefore Seebeck coefficient increases with increase in temperature[7] as shown in Figure 42 (b). Seebeck coefficient follows an inverse relationship with carrier concentration. 

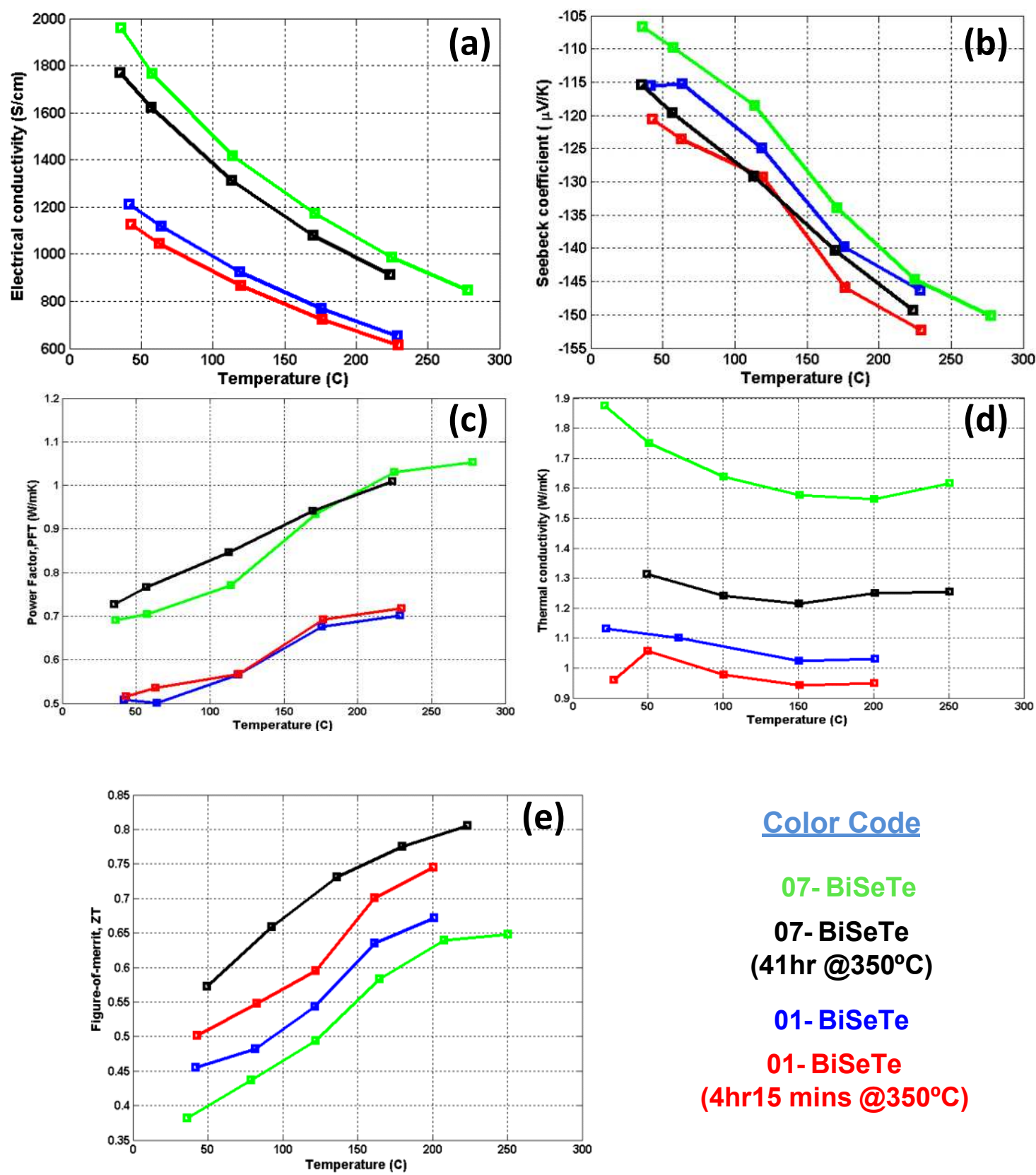

\section{Color Code}

\section{7-BiSeTe}

\section{7-BiSeTe (41hr@350 C)}

\section{1-BiSeTe}

01-BiSeTe

(4hr15 mins @350²)

Figure 42: Temperature dependence of (a) electrical conductivity, (b) Seebeck coefficient, (c) power factor, (d) thermal conductivity and (e) figure of merit of different powders and sample annealing time

As seen from Figure 42 (d), thermal conductivity decreases as the temperature increases and beyond certain temperature, increases with temperature. During heat transfer both the lattice and electronic part take place in thermal conductivity, but phonon heat transfer is more dominant at 
lower temperatures. As temperature increases, electronic parts become more prominent. During initial decrease in thermal conductivity, phonons contribute towards the thermal conductivity and during the increase, carriers contributes to thermal conductivity. The band gap of the material becomes narrow at high temperatures and thermal conductivity can happen from contribution of both holes and electrons. This effect is called "bipolar" and is very prominent at high temperatures as can be seen in Figure 42 (d) at temperatures above $200^{\circ} \mathrm{C}$.

Electrical and thermal conductivity of sample 07-BiSeTe-P2 is greater than that of sample 01BiSeTe-P1. This can be attributed to two reasons, powder of sample 07-BiSeTe-P2, underwent 2 cycles of long duration annealing, however final milling carried out was for only 5 hours hence sufficient grain size reduction would not have had happened. The pressing conditions of these samples were similar except for the total time taken for pressing, there was an increase of 2 minutes and 15 seconds. The grain size increased because of increase in total time taken for pressing the sample. The alloy powders become soft at high temperatures and tend to grow, this can happen after about $300^{\circ} \mathrm{C}$. Therefore, due to large grain size, we can account of the increase in thermal and electrical conductivity.

As the grain size grows, scattering will be low. Scattering is a phenomenon in which charge carriers and phonons deviate from their straight path trajectory, because of collision with each other, among each other or grain boundaries. As a result the mean free path of the carriers and phonons are reduced.

Upon annealing the samples at $350^{\circ} \mathrm{C}$, drop in electrical conductivity and thermal conductivity was observed for the samples as shown in Figure 42 (a) and (d). Annealing decreases the defects in the samples which leads to decease in the carries concentrations which in n-type materials are electrons. As a result Seebeck coefficient increases and electrical conductivity decreases. Mobility also increases with defect healing. The resultant thermal conductivity drops, owing to 
the well defined grain boundary formation during annealing temperatures for extended time. This leads to increase in power factor and thus enhances the figure of merit of the samples. This trend is observed for both samples of 01-BiSeTe-P1 and 07-BiSeTe-P2 in Figure 42 (d) and (e).

Power factor and figure of merit (ZT) also changes simultaneously with changes in thermoelectric properties. The highest ZT value was obtained for sample $07-\mathrm{BiSeTe}-\mathrm{P} 2-\mathrm{A} 2,0.8$ at $220^{\circ} \mathrm{C}$.

\subsubsection{Effect of holding/sintering time}

The effect of holding/sintering time, pressure and temperature can be explained from the plots from Figure 43.

The effect of increasing the holding time from zero to 90 seconds for samples 03- BiSeTe-P1 compared to $01-\mathrm{BiSeTe}-\mathrm{P} 1$ is shown by the increase in electrical and thermal conductivity, due to the growth in grain size. The slight difference in Seebeck coefficients of these samples can be attributed to the decrease in pressure from 400psi to 350psi and due to the 24 hours extra milling which causes variation in defect density. 

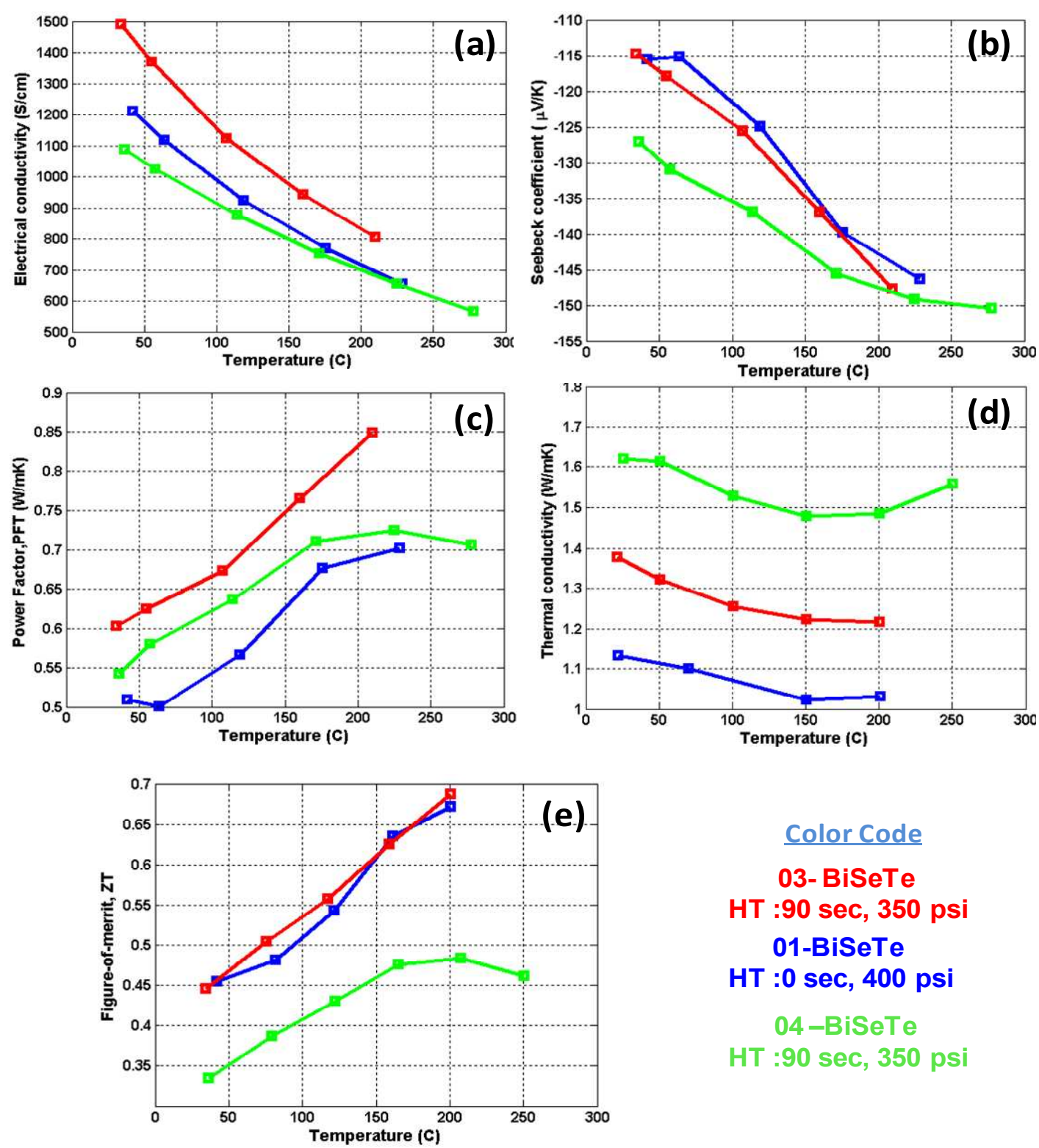

Color Code

03- BiSeTe

HT :90 sec, 350 psi

01-BiSeTe

HT :0 sec, 400 psi

$04-\mathrm{BiSeTe}$

HT :90 sec, 350 psi

Figure 43 : Temperature dependence of (a) electrical conductivity, (b) Seebeck coefficient and (c) power factor (d) thermal conductivity and (e) figure of merit of different samples showing effect of holding/sintering time, pressure and temperature

On comparison of sample 01- BiSeTe-P1 to 04- BiSeTe-P3, the carrier concentrations of sample are different because of annealing of powders at different conditions. The effect of decrease in 
pressure can be observed from the fact that electrical conductivity reduced. Seebeck coefficient was enhanced to a significant extent, which can be attributed to the decrease in pressure. The decrease in density decrease leads to more carriers. Thermal conductivity increase in sample 04BiSeTe-P3 can be attributed to the annealing of the powders. The grain size of the powders was increased during annealing and milling of the powders was only conducted for $4 \mathrm{hrs}$. Scattering reduced because of increase in grain size.

The effect of annealing powders and less milling can be best observed on comparison of samples 04-BiSeTe-P3 and 01- BiSeTe-P1. Thermal conductivity is increased to a higher magnitude, due to increase in grain size. Slight increase in power factor is observed, due to increase in mobility of carriers in sample 04-BiSeTe-P3. However, the loss on ZT compared to sample 01-BiSeTe-P1 and $03-\mathrm{BiSeTe}-\mathrm{P} 1$ is due to increase in thermal conductivity.

\subsubsection{Composite nanoparticle doping with Silicon Germanium Arsenide}

Silicon germanium arsenide is an n-type thermoelectric material used at high temperatures $\left(850^{\circ} \mathrm{C}-1000^{\circ} \mathrm{C}\right)$. Alloy of bismuth selenium telluride and silicon germanium arsenide was formed contemplating silicon germanium arsenate as a phonon scattering medium in the alloy powders. The plots for thermoelectric transport properties measured are shown in Figure 44.

Significant improvement of thermoelectric properties was expected, but measurement of Seebeck coefficient and electrical conductivity did not prove so. Electrical conductivity in Figure 44 (a) increased with increase in temperature, this behavior is typical of semiconductors and are not desirable for thermoelectric materials. 

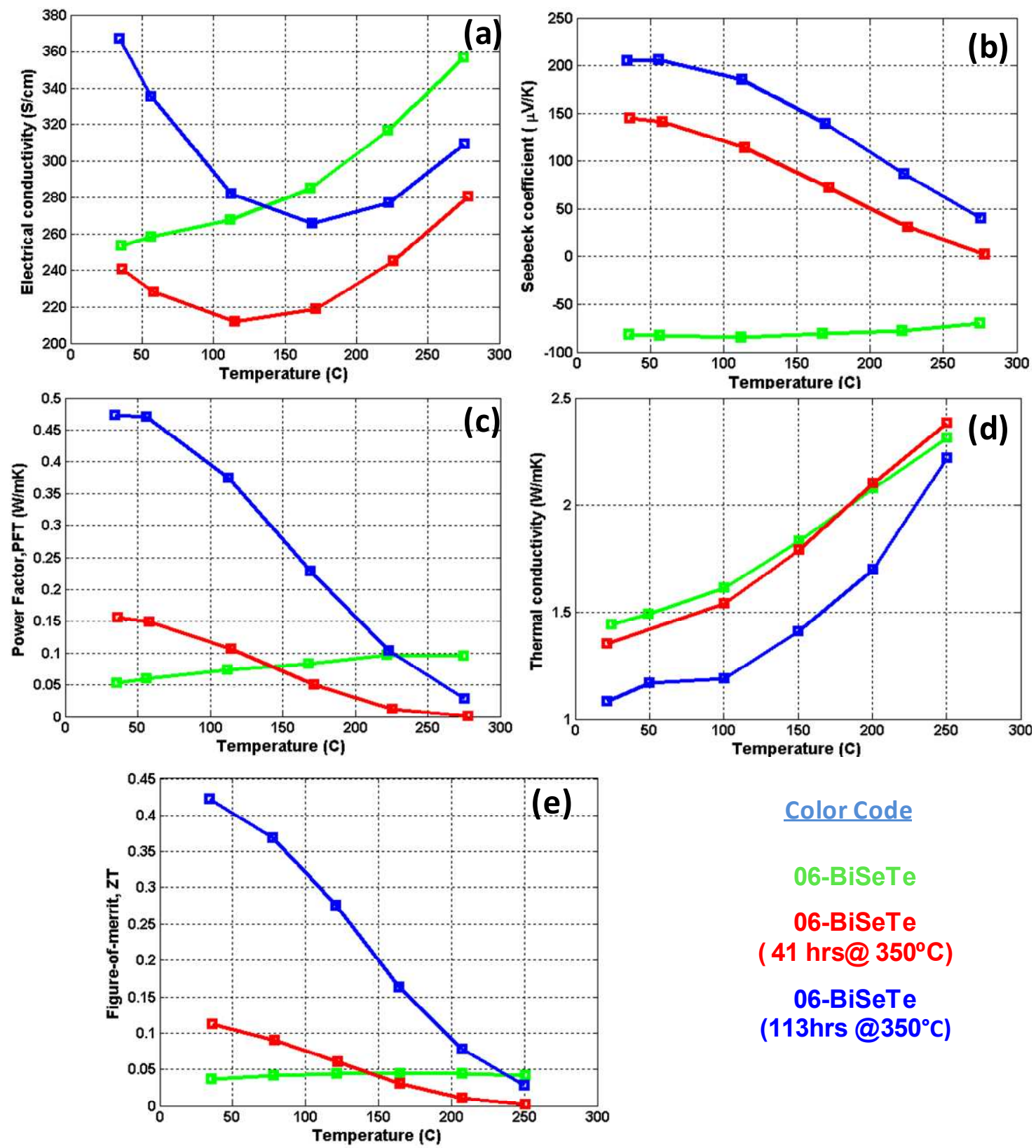

Color Code

06-BiSeTe

06-BiSeTe

(41 hrs@350 $\mathrm{C}$ )

06-BiSeTe

(113hrs@350ㄷ)

Figure 44: : Temperature dependence of (a) electrical conductivity, (b) Seebeck coefficient, (c) power factor, (d) thermal conductivity and (e) figure of merit of different powders and sample annealing time 
The samples were annealed at high temperature of $350^{\circ} \mathrm{C}$ for an extended time period of 41 hours and the measurements were repeated to study the effect of annealing the sample and to find any potential enhancement of the thermoelectric properties. The as pressed sample had shown n-type material characteristics, Seebeck co-efficient had negative magnitude, but upon annealing the characteristics of sample changed and displayed p-type material characteristics. The Seebeck coefficient became positive. The Seebeck coefficient changed from a negative value of 100 to a positive value of about 150 at $50^{\circ} \mathrm{C}$, similarly the electrical conductivity trend changed compared to the original sample.

To further investigate into this phenomenon, annealing of the sample was continued at $350^{\circ} \mathrm{C}$ for $72 \mathrm{hrs}$ more. The second cycle annealed sample measurements were made showed significant improvement in the thermoelectric properties. Gain in power factor as compared to the first annealing data. Between $25^{\circ} \mathrm{C}-200{ }^{\circ} \mathrm{C}$, there was power factor improvement, about three times at $25^{\circ} \mathrm{C}$. Thermal conductivity data also showed lowering. Following the similar trend for power factor, three times improvement was also seen in figure of merit.

The rationale behind this change, has yet to be found out. It can be attributed to possible formation of a new phase of alloy which has to be confirmed by XRD analysis.

These experiments showed that, inclusion of Silicon Germanium Arsenide into bismuth selenium telluride has potential of improving thermoelectric properties of bismuth selenium telluride. 


\section{CHAPTER VI}

\section{CONCLUSION, COST AND FUTURE WORK}

\subsection{Conclusion}

The project is aimed at developing thermoelectric devices for multifunctional energy harvesting structural composites.

- A novel method of fabricating nanostructured thick film thermoelectric devices suitable for thermoelectric energy harvesting composites was devised.

- Layered structure comprising of contact metal and thermoelectric materials was successfully fabricate as it overcomes the problem of diffusion and contact resistance when compared to regular thin films.

- This method is suitable for manufacturing thermoelectric devices owing to the ease and inexpensive fabrication techniques when compared to thin film devices.

- Thermoelectric p-type bismuth antimony telluride with diffusion barrier layer and metal layers were fabricated. Initial, work for the fabrication of n-type material was also carried out.

- Thermoelectric n-type bismuth selenium telluride samples were pressed and characterized as so as to optimize the powder alloying and pressing conditions for best figure of merit results. 
- A new composite nano doping mechanism of inclusion of silicon germanium arsenide into bismuth selenium telluride was conducted.

Thermoelectric transport property characterizations done on the p-type bismuth antimony telluride interlaminar layer, n-type bismuth selenium telluride and silicon germanium arsenide

nano composite doped bismuth selenium telluride proved to be a good starting point for making modules through the layered structure approach.

\subsection{Cost}

Preliminary calculations show that a 1 meter by 1 meter thermoelectric module can generate about 358 Watt power. This calculation is based on the fact that, a figure of merit of 1.57 can be achieved for the thermoelectric material of the proposed thermoelectric module.

The proposed thermoelectric device legs will have a square cross-section of $0.02 \mathrm{~cm}$ and $0.05 \mathrm{~cm}$ height, with leg spacing of $0.01 \mathrm{~cm}$. The cost of thermoelectric material with a density of $6.8 \mathrm{~g} / \mathrm{cm}^{3}$ required for the fabrication is about $170 \$ / \mathrm{kg}$.

Assuming hot and cold side temperatures to be about $150^{\circ} \mathrm{C}$ and $20^{\circ} \mathrm{C}$ with constant heat flow rate at $1 \mathrm{~W} / \mathrm{cm}^{2}$ for calculation purposes, the power generation in one single leg is $0.0000323 \mathrm{~W}$. Also assuming all of thermoelectric legs in a given area are functional and are subject to a constant and uniform temperature difference, the power generation per $\mathrm{cm}^{2}$ is $0.0363 \mathrm{~W} / \mathrm{cm}^{2}$. An efficiency of about $8 \%$ can be achieved with the current available thermoelectric technology.

The cost of production of such a device is estimated to be about $\$ 1.28 / \mathrm{cm}^{2}$.

\subsection{Future work}

Further optimizations of the alloy powders and pressing conditions could lead to improved figure of merit. The thickness of the metal layer, diffusion barrier and thermoelectric layer of layered 
structure could be optimized. Materials like Tantalum Silicon nitride layers can be used as barrier diffusion layer in thermoelectrics[40, 49].

Metal layer of copper can be replaced with other alloys which are compatible with thermoelectric materials. Alloy that can be considered for this process is Braze 560 alloy from Lucas Milhaupt. The composition of this alloy are, $56.0 \% \pm 1.0 \%$ Silver, $22.0 \% \pm 1.0 \%$ copper, $17.0 \% \pm 2.0 \%$ Zinc, $5.0 \% \pm 0.5 \%$ Tin and other elements $0.15 \%$. Silver metal being the main component of the alloy has greater electrical conductivity than copper at RT[50]. Therefore, this alloy can be used as replacement for the copper metal layer. Optimization of the thickness of the metal layer can be achieved and lesser material can be used for the manufacturing of the modules and thus compensate for the high cost of the silver alloy.

The n-type thermoelectric bismuth selenium telluride alloy formation and sample hot pressing processes can be optimized to obtain the highest $\mathrm{ZT}$ of 1.04 at $125^{\circ} \mathrm{C}$ obtained for these materials. Improved results could be obtained using composite powders approach used by Mehdizadeh et. al. [32]. for p-type thermoelectric bismuth antimony telluride. Multilayered sandwich structures for n-type thermoelectric could be fabricated in a similar manner as p-type thermoelectric material.

The second phase of the project would be processing the multilayered sandwich thermoelectric structure of p-type and n-type thermoelectrics, merging them together and creating a thermoelectric module.

The final phase of the project is incorporation and integration of these thermoelectric modules onto composites for the realization of multilayered thermoelectric structural composites for waste thermal energy harvesting. 


\section{REFERENCES}

[1] M. S. Dresselhaus and I. L. Thomas, "Alternative energy technologies," Nature, vol. 414, pp. 332-337, 2001.

[2] M. K. Hubbert, "Energy from Fossil Fuels," Science, vol. 109, pp. 103-109, February 4, 1949.

[3] S. Shafiee and E. Topal, "When will fossil fuel reserves be diminished?," Energy Policy, vol. 37, pp. 181-189, 2009.

[4] A. Midilli, et al., "Green energy strategies for sustainable development," Energy Policy, vol. 34, pp. 3623-3633, 2006.

[5] J. Hansen, et al., "Global warming in the twenty-first century: An alternative scenario," Proceedings of the National Academy of Sciences, vol. 97, pp. 9875-9880, August 29, 2000.

[6] J. P. Heremans, et al., "Thermoelectric Power of Bismuth Nanocomposites," Physical Review Letters, vol. 88, p. 216801, 2002.

[7] G. J. Snyder and E. S. Toberer, "Complex thermoelectric materials," Nat Mater, vol. 7, pp. 105-114, 2008.

[8] H. J. Goldsmid, Introduction to Thermoelectricity: Springer, 2009.

[9] T. J. Seebeck, "Magnetische Polarisation der Metalle und Erze durch TemperaturDifferenz," Abh. Akad. Wiss. Berlin, vol. 1820-21, pp. 289-346, 1822.

[10] M. S. Dresselhaus, et al., "New Directions for Low-Dimensional Thermoelectric Materials," Advanced Materials, vol. 19, pp. 1043-1053, 2007 
[11] B. Poudel, " A study on thermoelectric properties of Nanostructured Bulk Materials," Doctor of Philosopy, Department of Physics, Boston College, Boston, 2007.

[12] B. G. Streetman and S. Banerjee, Solid state electronic devices: Pearson Prentice Hall, 2006.

[13] C. Kittel, Introduction to solid state physics: Wiley, 2005.

[14] L. D. Hicks and M. S. Dresselhaus, "Effect of quantum-well structures on the thermoelectric figure of merit," Physical Review B, vol. 47, p. 12727, 1993.

[15] J. Laird, "SunShot takes aim at PV costs: Part two: With its SunShot program, the US Department of Energy (DoE) is trying to lead the world in research dedicated to slashing PV costs," Renewable Energy Focus, vol. 12, pp. 44-46, 48-49.

[16] p. P. H. J. Goldsrtlid, New York, ). Thermoelectric Refrigeration: Plenum Press, 1964.

[17] M. G. Kanatzidis, "Nanostructured Thermoelectrics: The New Paradigm?†," Chemistry of Materials, vol. 22, pp. 648-659, 2009.

[18] R. M. Jones, Mechanics of composite materials: Taylor \& Francis, 1999.

[19] D. D. L. Chung and S. Han, "multifunctional carbon fiber epoxy-matrix composites for energy harvesting."

[20] D. M. Rowe, CRC handbook of thermoelectrics: CRC Press, 1995.

[21] B. C. Sales, et al., "Filled skutterudite antimonides: Electron crystals and phonon glasses," Physical Review B, vol. 56, p. 15081, 1997.

[22] G. Chen, "Thermal conductivity and ballistic-phonon transport in the cross-plane direction of superlattices," Physical Review B, vol. 57, p. 14958, 1998.

[23] R. Venkatasubramanian, et al., "Thin-film thermoelectric devices with high roomtemperature figures of merit," Nature, vol. 413, pp. 597-602, 2001.

[24] T. C. Harman, et al., "Quantum Dot Superlattice Thermoelectric Materials and Devices," Science, vol. 297, pp. 2229-2232, September 27, 2002. 
[25] K. F. Hsu, et al., "Cubic AgPbmSbTe2+m: Bulk Thermoelectric Materials with High Figure of Merit," Science, vol. 303, pp. 818-821, February 6, 20042004.

[26] G. S. Nolas, et al., "The effect of rare-earth filling on the lattice thermal conductivity of skutterudites," Journal of Applied Physics, vol. 79, pp. 4002-4008, 1996.

[27] G. S. Nolas, et al., "Recent Developments in Bulk Thermoelectric Materials," MRS Bulletin, vol. 31, pp. 199-205 2006.

[28] S. M. Kauzlarich, et al., "Zintl phases for thermoelectric devices," Dalton Transactions, pp. 2099-2107, 2007.

[29] P. Pichanusakorn and P. Bandaru, "Nanostructured thermoelectrics," Materials Science and Engineering: R: Reports, vol. 67, pp. 19-63, 2010.

[30] Q. H. Bed Poudel, Yi Ma, Yucheng Lan, Austin Minnich, Bo Yu, Xiao Yan, Dezhi Wang, Andrew Muto, Daryoosh Vashaee, Xiaoyuan Chen, Junming Liu, Mildred S. Dresselhaus, Gang Chen, and Zhifeng Ren, "High-Thermoelectric Performance of Nanostructured Bismuth Antimony Telluride Bulk Alloys," Science, vol. 320, pp. 634638,2008

[31] X. Yan, et al., "Experimental Studies on Anisotropic Thermoelectric Properties and Structures of n-Type Bi2Te2.7Se0.3," Nano Letters, vol. 10, pp. 3373-3378, 2010.

[32] W. Xie, et al., "Unique nanostructures and enhanced thermoelectric performance of meltspun BiSbTe alloys," Applied Physics Letters, vol. 94, pp. 102111-3, 2009.

[33] S. Fan, et al., "p-type Bi[sub 0.4]Sb[sub 1.6] Te[sub 3] nanocomposites with enhanced figure of merit," Applied Physics Letters, vol. 96, pp. 182104-3, 2010.

[34] X. B. Zhao, et al., "Bismuth telluride nanotubes and the effects on the thermoelectric properties of nanotube-containing nanocomposites," Applied Physics Letters, vol. 86, pp. 062111-3, 2005.

[35] G. J. Snyder, et al., "Thermoelectric microdevice fabricated by a MEMS-like electrochemical process," Nat Mater, vol. 2, pp. 528-531, 2003. 
[36] D. J. Griffiths, Introduction to electrodynamics: Prentice Hall, 1999.

[37] R. O. Carlson, "Anisotropic diffusion of copper into bismuth telluride," Journal of Physics and Chemistry of Solids, vol. 13, pp. 65-70, 1960.

[38] B. M. Goltsman, V. A. Kudinov, I. A. Smirnov, Semiconducting thermoelectric materials based on Bi2Te3, Nauka,1972.

[39] Y. C. Lan, et al., "Diffusion of nickel and tin in p-type (Bi,Sb)[sub 2]Te[sub 3] and ntype $\mathrm{Bi}[\mathrm{sub} 2](\mathrm{Te}, \mathrm{Se})[\mathrm{sub} 3]$ thermoelectric materials nanocrystallization of amorphousTa[sub 40]Si[sub 14]N[sub 46] diffusion barrier thin films," Applied Physics Letters, vol. 92, pp. 101910-3, 2008.

[40] I. V. Gasenkova and T. E. Svechnikova, "Structural and Transport Properties of SnDoped Bi2Te3 - xSex Single Crystals," Inorganic Materials, vol. 40, pp. 570-575, 2004.

[41] O. D. Iyore, et al., "Interface characterization of nickel contacts to bulk bismuth tellurium selenide," Surface and Interface Analysis, vol. 41, pp. 440-444, 2009.

[42] H. Bottner, et al., "New thermoelectric components using microsystem technologies," Microelectromechanical Systems, Journal of, vol. 13, pp. 414-420, 2004.

[43] H. Ernst, et al., "High temperature stable contacts for thermoelectric sensors and devices," in Thermoelectrics, 2001. Proceedings ICT 2001. XX International Conference on, 2001, pp. 521-524.

[44] B. D. Cullity and S. R. Stock, Elements of x-ray diffraction: Prentice Hall, 2001.

[45] J. C. K. Ranji Vaidyanathan, "LIGHTNING STRIKE MITIGATION COMPOSITES," USA Patent, 2009.

[46] Z. Z. A Mehdizadeh Dehkordi, Xinghua Shi and Daryoosh Vashaee, "Title," unpublished|.

[47] R. Martin-Lopez, et al., "Preparation of n-type Bi-Sb-Te thermoelectric material by mechanical alloying," Solid State Communications, vol. 108, pp. 285-288, 1998. 
[48] A. F. Ioffe, Semiconductor thermoelements, and Thermoelectric cooling: Infosearch, 1td., 1957.

[49] D. R. Lide, CRC handbook of chemistry and physics: a ready-reference book of chemical and physical data: CRC Press, 2004.

[50] M. Bicker, et al., "Nanocrystallization of amorphous-Ta[sub 40]Si[sub 14]N[sub 46] diffusion barrier thin films," Applied Physics Letters, vol. 78, pp. 3618-3620, 2001.

[51] R. A. Serway and Jewett, J.W., Principles of Physics: Cengage Learning, 2003.

[52] R. Murugesan, et al., "Multi-faceted role of blended poly(vinyl pyrrolidone) leading to remarkable improvement in characteristics of polyaniline emeraldine salt," Materials Chemistry and Physics, vol. 85, pp. 184-194, 2004. 


\title{
APPPENDICES
}

\author{
APPENDIX A
}

\section{A.1 Direct blending}

Based on a standalone film based technology development, where CNT (Carbon Nano Tubes) dispersed in PVP (Polyvinylpyrrolidone) solution could be blended [51], a crude method of trying to disperse the thermoelectric powders in PVP solution was tried out.

\section{A.1.1 Experimental Procedures}

PVP was soluble in ethyl alcohol. Uniform blend of PVP ethyl alcohol solvent was obtained upon mixing with a magnetic stirrer. This solution on pouring on a flat surface leaves behind a PVP standalone film upon evaporation of the ethyl alcohol. The amount of PVP K-60 powder used was fixed. PVP K-60, reagent standard ethyl alcohol were purchased from Sigma-Aldrich and was used for all the experiments.

Thermoelectric powders of p-type bismuth antimony telluride synthesized in the methods mentioned in chapter IV, were tried to blend into the PVP solution in many ways. Varying from simple hand mixing using a glass rod, magnetic stirrer to ultra sonicator blending was also tried. The mixed PVP blend and thermoelectric powder mix was carefully poured onto glass slides and allowed to dry in the fume hood for 12 hours. Before pouring the final blend onto the glass slides, 
a water based wax was applied on the glass slide for easy detachment of the dried film from the glass slide.

Hexane was tried as a solvent, but PVP failed to dissolved and form a solution. In order for the TE powder to uniformly disperse in the polymer PVP, SDS (Sodium dodecyl sulfate) surfactant was used. SDS was used as a wetting agent to decrease the surface tension of the solvent and to better enhance the interactional behavior between the TE powder and the PVP solvent blend.

Since, the TE properties depend on the concentration of the TE powders in the PVP blend, the doping level of the TE powders in the polymer was varied. Three different doping levels were taken into consideration. For the corresponding amount of TE powder used, the amount of SDS surfactant to be used was calculated. The three doping concentrations considered are shown in Table 3

Table 3: Different doping levels considered for thermoelectric polymer thin films

\begin{tabular}{llll}
\hline Material & Low doping (gms) & Medium doping(gms) & High doping (gms) \\
\hline BiSbTe & 0.14 & 1.4 & 4.2 \\
SDS & 0.0576 & 0.576 & 1.728 \\
PVP & 0.86 & 0.86 & 0.86 \\
\hline
\end{tabular}

Carbon black powder was used as an agent to enhance the electrical properties. A conducting polymer like polyanilne (PANI) was replaced with PVP as conducting substrate in later experiments.

\section{A.1.2 Results and Discussions}

The films created showed uneven conductivity when measured by using a regular multimeter at different areas. However, some areas on the film showed conductivity, but it was attributed to 
thermoelectric powders being concentrated on a particular area. Figure 45 shows the picture of blended thermoelectric polymer film on glass plate.

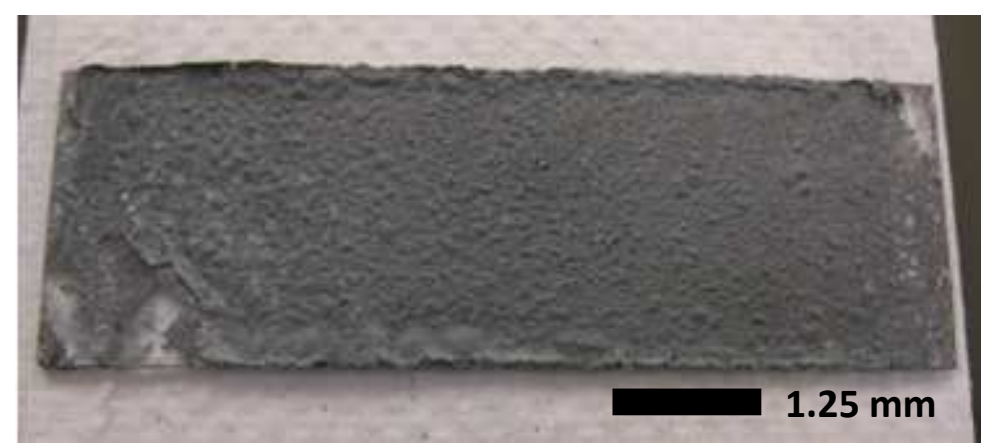

Figure 45 : Picture of film dried on glass plate

To mention some random electrical resistance of the films measured, using a multimeter for the different doping concentrations vary from $104.6 \mathrm{k} \Omega$ for medium doped film to $4.34 \mathrm{M} \Omega$ for the heavily doped film

During the blending and mixing operation of the PVP solution and thermoelectric powder, the powders sunk to the bottom of the blend because of its larger mass. Same case remained even after the various methods of the blending. The blending time using ultra-sonication was varied in between 5 minutes to 30 minutes continuous operation. The concentration of the thermoelectric powders was maximum in some areas and minimum in the others. This explains the electrical conductivity peaks in some areas and lack of conductivity in the other areas.

Also, other reason that can explain the loss in electrical conductivity is the oxidation of the TE powders. These powders are sensitive to oxygen and rapidly oxidize, decreasing the electrical conductivity. Carbon black powder was also used as an enhancing agent during the time of ultrasonication mixing, to improve the electrical conductivity. A measured and constant amount of 
$0.01 \mathrm{gm}$ of carbon was also added to all the various film concentrations varying from least doped to heavily doped. An addition of the carbon black did not improve the electrical conductivity.

Since, a conductive base or substrate to disperse the thermoelectric powders was needed, PVP was replaced with Polyanilne (PANI) solution. Same procedure was conducted for making the PVP based thermoelectric film was followed, for blending PANI and thermoelectric powders together. No improvement was noticed after addition of thermoelectric powder when measured by a multimeter.

\section{A.2 Polymerization}

Another approach was to fabricate PVP- PANI blend as carrier for the thermoelectric powders.

Blending PVP into the aniline enhances the electrical characteristics[52]. The PVP-ANI(aniline) mixture was polymerized using the chemical oxidative polymerization reaction. Thermoelectric powders were introduced into the PVP- ANI mixture before the polymerization reaction, to create the PVP-PANI-thermoelectric powder or solution. At the end of the reaction the polymer powders with the thermoelectric powders were filtered out.

The processed thermoelectric powders were mixed with ethanol. to create a film on a glass plate. The objective was to create a standalone film, but the mixture did not dissolve in the ethyl alcohol solution.

\section{A.3 Conclusion}

Limited experiments were conducted to fabricate thermoelectric polymer films was conducted. Further study needs to be focused in this direction to obtain more promising results. 


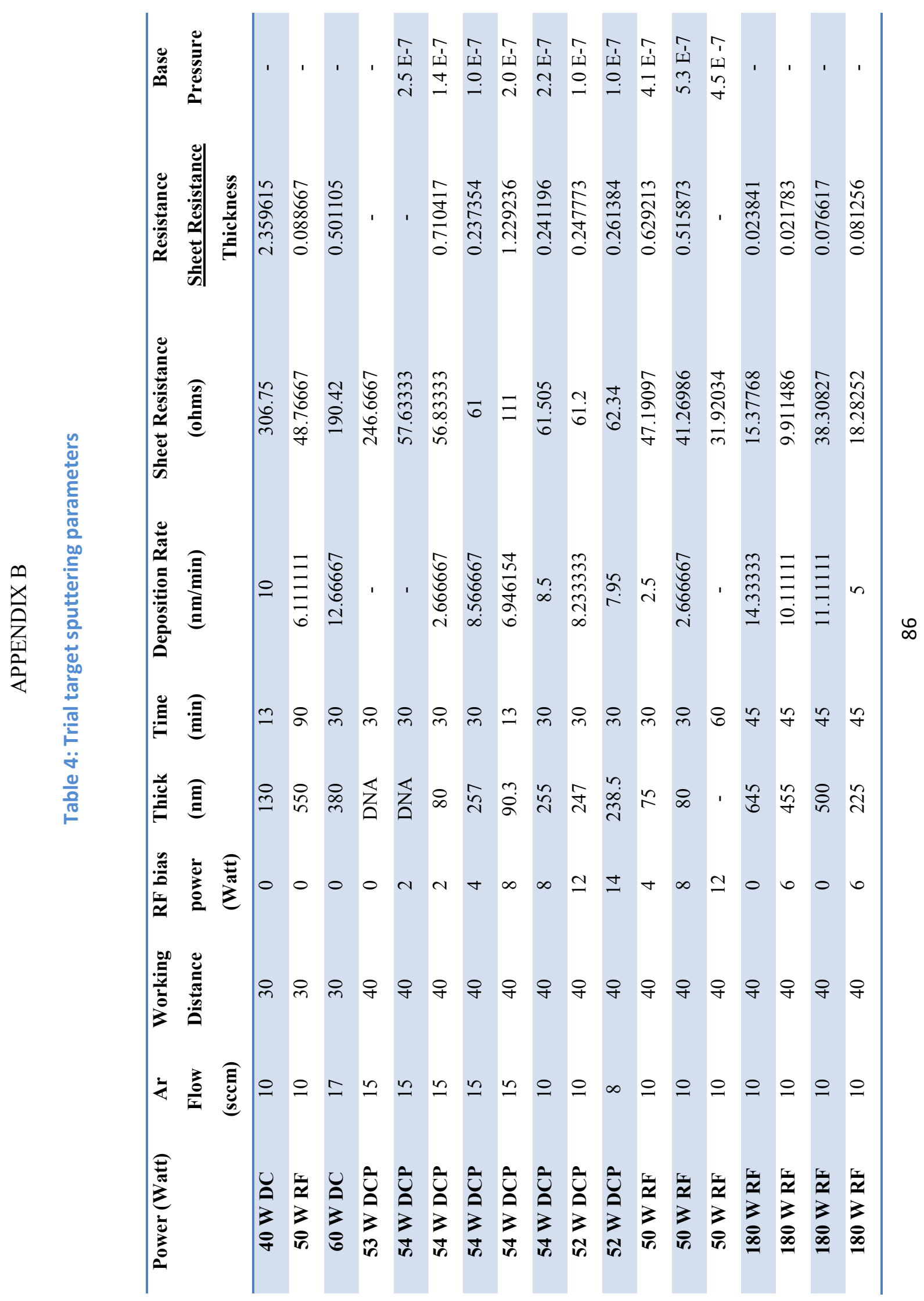


VITA

Oonnittan Jacob Panachaveettil

Candidate for the Degree of

Master of Science

Thesis: DEVELOPMENT OF THERMOELECTRIC DEVICES FOR STRUCTURAL COMPOSITES

Major Field: Mechanical and Aerospace Engineering

Biographical:

Education:

Completed the requirements for the Master of Science/Arts in your major at Oklahoma State University, Stillwater, Oklahoma in December,2011.

Received Bachelor of Science Degree in Mechanical Engineering at Mahatma Gandhi University, India in 2007.

Experience: Thin film deposition, Bulk thermoelectrics materials

Professional Memberships: SAE 
Name: Oonnittan Jacob Panachaveettil

Date of Degree: December, 2011

Institution: Oklahoma State University

Location: Stillwater, Oklahoma

Title of Study: DEVELOPMENT OF THERMOELECTRICS DEVICES FOR STRUCTURAL COMPOSITES

Pages in Study: 87

Candidate for the Degree of Master of Science

Major Field: Mechanical and Aerospace Engineering

Scope and Method of Study:

The study presented in this thesis covers a multiple methods for manufacturing thermoelectric structural composites for waste heat energy harvesting efficiently. Various attempts to fabricate a thermoelectric device suitable for incorporation into composites giving a entirely new dimension of harvesting waste energy is sought. A brief study into making thermoelectric materials of high figure of merit was also conducted as a part of the project.

Findings and Conclusions:

A novel method of fabricating thick film thermoelectric devices which can be mounted onto composites was devised and conceptualized. The implementation of this device has been carried out partially. This is method surpasses the thin film thermoelectric device process when compared to the efficiency and manufacturing cost. A process using a three layered structure consisting of metal contacts and TE materials that are sintered and diced into thick films for device fabrication. This method can be extended to other TE materials irrespective of the temperature range.

ADVISER'S APPROVAL: Dr. Kaan Kalkan 